\title{
Are fish sensitive to trawling recovering in the Northeast Atlantic?
}

\author{
Rindorf, Anna; Gislason, Henrik; Burns, Finlay; Ellis, Jim R.; Reid, David
}

Published in:

Journal of Applied Ecology

Link to article, DOI:

10.1111/1365-2664.13693

Publication date:

2020

Document Version

Peer reviewed version

Link back to DTU Orbit

Citation (APA):

Rindorf, A., Gislason, H., Burns, F., Ellis, J. R., \& Reid, D. (2020). Are fish sensitive to trawling recovering in the Northeast Atlantic? Journal of Applied Ecology, 57(10), 1936-1947. https://doi.org/10.1111/1365-2664.13693

\section{General rights}

Copyright and moral rights for the publications made accessible in the public portal are retained by the authors and/or other copyright owners and it is a condition of accessing publications that users recognise and abide by the legal requirements associated with these rights.

- Users may download and print one copy of any publication from the public portal for the purpose of private study or research.

- You may not further distribute the material or use it for any profit-making activity or commercial gain

- You may freely distribute the URL identifying the publication in the public portal 
1 Are fish sensitive to trawling recovering in the Northeast Atlantic?

2 Anna Rindorf

3 National Institute of Aquatic Resources

4 Danish Technical University

5 Kemitorvet, Building 201

62800 Kgs. Lyngby

7 Denmark

$8 \quad$ ar@aqua.dtu.dk, tel. +4535883378

9

10 Henrik Gislason

11 National Institute of Aquatic Resources

12 Danish Technical University

13 Kemitorvet, Building 201

142800 Kgs. Lyngby

15 Denmark

16

17 Finlay Burns

18 Marine Scotland Science

19375 Victoria Road

20 Aberdeen AB11 9DB

21 UK

23 Jim R Ellis

24 Centre for Environment, Fisheries and Aquaculture Science 
25 Pakefield Road

26 Lowestoft

27 Suffolk

$28 \quad$ UK

29

30 David Reid

31 Marine Institute

32 Rinville, Oranmore

33 Co. Galway, H91 R673

$34 \quad$ Ireland

35

36

37

1. Abstract

38

39 1. The protection of sensitive species from overfishing is a key aspect of the ecosystem

$40 \quad$ approach to fisheries management.

2. We use life history parameters and knowledge of fish shape and habitat to estimate the sensitivity of 270 species in the Northeast Atlantic to demersal trawling and compare sensitivity to the most recent IUCN categorization. Species classified as threatened were on average significantly more sensitive to trawling than other species. Using trawl surveys in European Atlantic waters from $36^{\circ} \mathrm{N}$ to $62^{\circ} \mathrm{N}$, we estimated indicators of abundance of 31 highly sensitive species and compared changes in abundance to

47 sensitivity, management measures and value of landings.

3. The abundance of 23 of the 31 sensitive species increased after year 2000 with 14 of the 49 species showing increases significant at the $5 \%$ level. The increases were not due to 
50 specific management measures, as less than half of the species were covered by catch

51 limits. Further, sensitivity or value of landings were not related to trends in abundance.

52 4. Three species (Atlantic wolf-fish, tusk and starry ray) declined significantly. These

$53 \quad$ species are all at their southern distributional limit in the North Sea.

54 5. Synthesis and applications. We recommend monitoring the development of sensitive

55 species to identify species under pressure and allow rapid management actions before

56 species enter the IUCN threatened category. Further, we recommend taking precautions

57 where species are under combined pressure from climate change and fishing.

59 Keywords: Sensitive fish, abundance indices, species recovery, IUCN categories, fisheries

60 management, life history traits, fish distributions

61 
63 A key aspect of the ecosystem approach to fisheries management is the protection of species

64 from overfishing (CBD, 2014). However, managing every species in large marine ecosystems is impractical and, therefore, approaches such as risk-based assessments (Hobday et al., 2011) have been proposed to ensure that the most sensitive components are the focus of management. A scoping process identifies priority objectives (Hobday et al., 2011), and a subsequent Population Susceptibility Analysis (PSA) or similar is used to identify the most susceptible species. In Europe, priority objectives stated in the Marine Strategy Framework Directive (MSFD) aim to support 'halting biodiversity loss, ensuring the conservation and sustainable use of marine biodiversity' (European Commission, 2008). These objectives have been confirmed in various large-scale scoping exercises (Rindorf et al., 2017).

The Minimum Viable Population concept is often used in viability analysis of terrestrial populations (Beissinger \& McCullough, 2002) and for marine fish populations, the minimum spawning stock per recruit concept plays a similar role. Basically, fishing should not reduce the spawning stock per recruit of a fish population below the minimum level necessary for replacement. For fish, this level is typically in the order of 20 to $40 \%$ of the unexploited population (Brooks et al., 2010). Large, slow growing, late maturing fish species with a low level of natural mortality are likely to reach the minimum level at a lower level of fishing mortality than small, fast growing, early maturing species with higher levels of natural mortality (Jennings et al., 1999; Fernandes et al., 2017). Life history characteristics such as asymptotic length $L_{\infty}$, growth rate $K$, the length at which $50 \%$ of the individuals have reached maturity $L_{\text {mat }}$, and natural mortality $M$ are therefore often used to indicate the sensitivity of different species to fishing (Hobday et al., 2011; Greenstreet et al., 2012). When speciesspecific information is missing, an estimate of the maximum length $L_{\max }$ of a species can be 
used to infer asymptotic length, growth rate, natural mortality, and proportion mature at length (Walker et al., 2019).

Following this approach, Le Quesne and Jennings (2012) used a life history model and asymptotic length to predict the sensitivity of different fish species in the Celtic Sea to fishing by estimating the fishing mortality required to reduce the stock to a specific level of spawning stock biomass per recruit relative to the unfished status, using a general relationship between size of the individual and the size selectivity in the fishery. However, in a subsequent study, gear efficiency expressed as the probability of being retained by a particular gear, was shown to have a larger effect on the estimated reference points than the uncertainty associated with general life history relationships (García-Carreras et al., 2015). Gear efficiency depends on the habitat, individual size, body shape and behavior of the fish (Table S1), as well as the properties of the gear (Reid et al., 2007). In 2010-2012, bottom trawl and beam trawl fisheries accounted for $98 \%$ of the discards in EU member countries (Catchpole et al., 2017). Hence, while bycatches taken in other fisheries may be important for

100 particular species, the probability of being retained in, or killed by, the bottom trawl and 101 beam trawl fisheries is likely to be most important for demersal fish. The objective of this study is to identify species sensitive to fishing in the Northeast Atlantic and provide an overview of changes in their abundance in the light of recent effort changes and current management. To achieve this aim, we ranked fish species according to their sensitivity to bottom and beam trawling in the Northeast Atlantic using gear efficiencies estimated by Walker et al. (2017). We then investigated whether species sensitivity was related to IUCN species status (Nieto et al., 2015), and whether management measures are in place to limit the impact of fishing on sensitive species. Finally, we examined the temporal development in the abundance of sensitive species in survey catches throughout the Northeast 
110 Atlantic and compared changes in abundance to sensitivity, current management measures,

111 the decreases in fishing effort from 2000 onwards and market value of the species.

Species sensitivity

115 Species sensitivity to trawl generated mortality was estimated by applying a length-based

116 Spawning stock per Recruit model (Beverton \& Holt, 1957; Gislason et al., 2008) to species

117 recorded in the DATRAS survey database covering the Northeast Atlantic from the coast of

118 Portugal to the North Sea and from the Baltic Sea to west of Scotland

119 (http://www.ices.dk/marine-data/data-portals/Pages/DATRAS.aspx). The maximum length of

120 the species, $L_{\max }$, the asymptotic length, $L_{\infty}$, the von Bertalanffy growth rate parameter, $K$,

121 length at first maturity, $L_{\text {mat }}$, and the length at which the individuals entered the sensitivity model (length at birth for chondrichthyan and length at metamorphosis for bony fish), $L_{\min }$, were derived from the literature whenever possible (Tables S2 and S3), thereby minimizing the variation induced by using one parameter to predict the remaining parameters whenever

125 possible (Thorson et al., 2014).

126 General relationships between life history parameters and species lengths were used to

127 provide missing values whenever information could not be found in the literature (Table

128 1). When no growth parameters were available, $L_{\infty}$ was estimated from $L_{\max }$ using the equation provided by Froese \& Binohlan (2000), $K$ was estimated from a regression of $\ln (K)$

130 versus $\ln \left(L_{\infty}\right)$ based on the species in the dataset for which $K$ and $L_{\infty}$ estimates were available $\left(\mathrm{p}<0.0001\right.$, adj. $\left.\mathrm{R}^{2}=0.43, \mathrm{df}=186\right)$. When $L_{\text {mat }}$ was missing, a regression of $\ln \left(L_{\text {mat }}\right)$ versus $\ln \left(L_{\infty}\right)\left(\mathrm{p}<0.0001, \operatorname{adj} \cdot \mathrm{R}^{2}=0.91, \mathrm{df}=155\right)$ based on species for which estimates were available was used to derive the missing value. For chondrichthyans,

134 regressions of $\ln \left(L_{\text {min }}\right)$ versus $\ln \left(L_{\infty}\right)$ differed significantly between egg-laying and live- 
135 bearing species (ANOVA, $\mathrm{P}(>\mathrm{F})=0.0004, \mathrm{n}=21$ ). Separate regressions of $\ln \left(L_{\text {min }}\right)$ versus

$136 \ln \left(L_{\infty}\right)$ were therefore used for egg-laying $\left(\mathrm{R}^{2}=0.68, \mathrm{df}=8\right)$ and live-bearing species $\left(\mathrm{R}^{2}=\right.$

$1370.81, \mathrm{df}=10)$. For bony fish, insufficient information about length at metamorphosis was

138 available and an overall $L_{\min }$ of $2 \mathrm{~cm}$ was adopted. The life history relationships were

139 entered into the model of Spawning Stock Biomass per recruit. The model was insensitive to

140 changes in the value of $L_{\min }$ as long as $L_{\min }$ was below the length at which the species is

141 either exploited or mature.

142 Bottom and beam trawls retain a variable proportion of the fish found in the path of the gear.

143 Some species, such as shallow water or reef-associated species, are difficult to catch because

144 their habitat is not consistently sampled during trawl surveys, while pelagic species, such as

145 Atlantic herring Clupea harengus are found in the water column and may pass above the

146 headline of the gear without being caught. Furthermore, among the individuals that enter the

147 mouth of the gear, the smaller individuals and species may escape through the meshes.

148 Towed gears will therefore generate species and size specific fishing mortalities in the area

149 where they operate. Walker et al. (2017) estimated the relative efficiency by which different

150 species groups and sizes of fish were retained by the major towed commercial fishing gears

151 operating in the North Sea. Dividing the species according to their body shape and typical

152 vertical position in the water column, catch efficiency at length (exploitation pattern) was

153 calculated for seven groups (Table S1). We allocated the species to these groups and used the

154 average efficiency of small and large meshed commercial beam trawls and otter trawls

155 estimated by Walker et al. (2017) to provide the relative catch efficiency for each $\mathrm{cm}$ of

156 length for each of the seven groups. The resulting exploitation pattern was used to estimate

157 the relative efficiency of towed gears for each of the species and size groups and provided the

158 relative fishing mortality at size by which they would be affected for a given level of overall

159 effort. 
160 As a measure of the sensitivity of each species to fisheries generated mortality, we used the

161 relative level of effort needed to reduce the biomass of mature individuals (the spawning 162 stock biomass) to $25 \%$ of its unfished level, $F_{25 \% S S B}$, (Table S2). Sensitive species can only

163 tolerate relatively low levels of fishing mortality before their spawning biomass is reduced to

$16425 \%$ of the unfished level. The lower the $F_{25 \% S S B}$, the higher the sensitivity. The exact

165 percentage (25\%) is of little consequence to the subsequent ranking, as the different $\mathrm{F}$

166 indicators are highly correlated (Le Quesne and Jennings, 2012). To distinguish between

167 sensitive and non-sensitive species, we used the $F_{25 \% S S B}$ of the most sensitive of the

168 commercial target species which has shown sustained high catches. Less sensitive species

169 may also be severely impacted by a targeted fishing effort, but here we concentrated on

170 species which are mostly bycaught in mixed fisheries rather than targeted directly. Ana- and

171 catadromous species were excluded from the study.

172

Comparison of sensitivities and IUCN ratings, $L_{\infty}$ and taxonomy

174 The estimated values of $F_{25 \% S S B}$ were analysed to determine if $F_{25 \% S S B}$ differed significantly

175 between IUCN ratings (ANOVA) and if there was a linear effect of asymptotic length $\left(L_{\infty}\right)$

176 and a categorical effect of taxonomy (Chondrichthyes, Agnatha and Actinopterygia) in a

177 generalized linear model with a linear effect of $L_{\infty}$ and a categorical effect of taxonomy. The

178 natural $\log$ of $F_{25 \% S S B}$ was taken prior to analyses as variance increased with the mean

179 of $F_{25 \% S S B}$.

180

181 Management of sensitive species in the EU and value of landing

182 Current management measures for EU fleets were derived from the latest available decisions

183 on fishing opportunities (European Commission, 2018a, b). Price per $\mathrm{kg}$ was derived from the

184 European Market Observatory for Fisheries and Aquaculture Products (www.eumofa.eu, 
accessed August $12^{\text {th }}$ 2019) and supplemented where prices were lacking with prices from

186 Hanstholm Auction (www.hanstholmfiskeauktion.dk/prices, accessed August 12 ${ }^{\text {th }} 2019$ ).

187 Species were ranked as high value if their value exceeded that of Atlantic cod Gadus morhua,

188 medium value if their value was between $50 \%$ and $100 \%$ of that of Atlantic cod, and low

189 value if their value was not listed or was less than $50 \%$ of that of Atlantic cod.

190

\section{Development in abundance of sensitive species}

192 Species abundance indices were derived from average survey catch rates in the ICES coordinated international bottom and beam trawl surveys in the area in which the species has historically been reported. Data were downloaded from the ICES DATRAS database (http://www.ices.dk/marine-data/data-portals/Pages/DATRAS.aspx $)$ on July $9^{\text {th }} 2019$. The

196 time-range for each survey are given in Table S4.

197 For the Baltic Sea, North Sea and west of Scotland, only hauls in which all fish species caught were recorded were analysed. Catch rates of beam trawls in the North Sea, where several beam widths are used, were standardised to a beam width of $4 \mathrm{~m}$. In the Baltic Sea, catches with the large and small versions of the trawl were considered as two different

201 surveys.

202 Species which were not accurately identified to species were joined in species groups.

203 Among the sensitive species, this affected the common skate complex (comprising records for Dipturus spp., D. batis, the invalid synonym D. flossada, and D. intermedius) and smooth-hounds (Mustelus asterias and M. mustelus). Annual estimates of mean catch rates were estimated for species observed in at least $50 \%$ of the years. The estimated mean

207 included only hauls taken in realised habitat defined as all ICES statistical rectangles in which the species had been recorded during a survey at any time in the data. Consequently, the total number of hauls varied between species. 
210 To provide integrated species indicators, two types of difference between surveys were

211 considered: differences in mean catch rate and differences in interannual variation in catch

212 rate. Differences in mean catch were accounted for by scaling all survey catches to a mean of

2131 in the years from 2009 onwards. Species-level indicators were derived by estimating the

214 weighted average of all indicators for the given species. As the study was focused on

215 population abundance indicators for long lived species, we expect a smooth development of

216 the underlying population size over the years. Survey catches may appear more or less

217 smooth, depending on the consistency of the catchability and relative local abundance of the

218 species in the particular survey. To ensure that more weight was placed on surveys showing

219 consistent population development, the combined indicators were weighted with the

220 reciprocal variance around a Loess smoother in time fitted to the logged non-zero catch rates

221 of each species in each survey. Further, to ensure a minimum precision level for the derived

222 indicators, combinations of surveys and species for which the CV of the residuals from the

223 Loess smoother exceeded 0.75 were excluded. Years in which the catch rates exceeded five

224 times the long-term mean were excluded in the analysis of integrated indicators. This

225 removed occasional very large hauls, which occurred for some of the species (e.g. Raja

226 clavata and Squalus acanthias). Indicators were integrated by region and across all regions.

227 After a period of increasing or stable high fishing pressure, demersal fishing effort in the

228 Northeast Atlantic area has decreased since the 2000s (ICES, 2018a, b, c). We therefore

229 estimated the trends in species abundance from 1980-2000 and from 2000 onwards as the

230 linear trends in log-transformed species-level indicators. In addition, the number of surveys

231 showing positive trends in the two periods was investigated (regardless of significance level).

232 The categorical effect on trend in abundance of value (low, medium or high) and

233 management (species TAC, group TAC or no TAC) was investigated in an ANOVA. 
237 The estimated values of $F_{25 \% S S B}$ for all 270 taxa are given in Table S2. The sensitivities of

238 species from Greenstreet et al. (2012) and Le Quesne and Jennings (2012) were well

239 correlated with those derived by the present method (Correlations $=-0.78$ and 0.74 ,

240 respectively, on a log-log scale). Some of the difference between the ratings can be attributed

241 to differences in the assumed values of the life history parameters. For example, Greenstreet

242 et al. (2012) assumed $L_{\infty}$ of spurdog Squalus acanthias to be $90 \mathrm{~cm}$, whereas Le Quesne and

243 Jennings (2012) and this study assumed 120 and $121 \mathrm{~cm}$, respectively. The most sensitive of

244 the commercial target species which has shown sustained high catches was saithe Pollachius

245 virens, with a value of $F_{25 \% S S B}$ of 0.43 . Using this species to define the level below which

246 species can be considered particularly sensitive, 59 species with $F_{25 \% S S B} \leq 0.43$ were defined

247 as sensitive species/taxa and used in subsequent analyses. These species were dominated by

24833 chondrichthyan taxa, including skates (Rajidae; Amblyraja hyperborea, A. radiata,

249 common skate-complex, D. nidarosiensis, D. oxyrinchus, Leucoraja circularis, L. fullonica,

250 L. naevus, Raja brachyura, $R$. clavata, $R$. microocellata, $R$. montagui, $R$. undulata, Rajella

251 bathyphila, Rajella fyllae, Rajella lintea and Rostroraja alba), squaliform sharks (Somniosus

252 microcephalus, Deania calcea, Squalus acanthias, Dalatias licha and Etmopterus princeps),

253 catsharks (Scyliorhinidae; Galeus melastomus, Scyliorhinus canicula and S. stellaris), hound

254 sharks (Triakidae; Galeorhinus galeus and Mustelus spp.) and various other species

255 (Hexanchus griseus, Lamna nasus, Torpedo marmorata, T. nobiliana, Dasyatis pastinaca, D.

256 tortonesei and Chimaera monstrosa). The remaining species comprised a variety of

257 gadiforms (Brosme brosme, Coryphaenoides rupestris, Macrourus berglax, Molva molva, $M$.

258 dypterygia, M. macropthalma, Mora moro and Phycis blennoides) and other species (Conger

259 conger, Lophius budegassa, L. piscatorius, Brama brama, Argyrosomus regius, Anarhichas 
lupus, A. minor, Dicentrarchus punctatus, Ephippion guttifer, Epigonus telescopus, Sebastes spp., Lepidorhombus whiffiagonis, Hippoglossus hippoglossus, Polyprion americanus,

Scophthalmus rhombus, Scorpaena scrofa and Synaphobranchus kaupi).

Comparing $F_{25 \% S S B}$ to the IUCN assessments, species sensitive to fishing occurred in all

IUCN categories. However, average $F_{25 \% S S B}$ was significantly higher for species in the Least

Concern category $(\mathrm{P}<0.0001)$ (Figure 1) indicating that less sensitive species were, on average, more likely to be categorized as Least Concern. There was no significant difference in the average $F_{25 \% S S B}$ between the Critically Endangered, Endangered, Vulnerable and Near Threatened categories $(\mathrm{P}=0.2594)$. None of the species in the Critically Endangered and Endangered categories showed an $F_{25 \% S S B}>0.75$ (Figure 2, Table S2). The two species classified as Endangered but not sensitive to demersal fishing pressure were basking shark Cetorhinus maximus and thresher shark Alopias vulpinus. Six Data Deficient species (Anarhichas lupus, Argyrosomus regius, Dasyatis tortonesei, Ephippion guttifer, Epigonus telescopus and Phycis blennoides) were identified as sensitive species. For the remaining 51 sensitive species, 24 are currently listed by the IUCN as Least Concern. variation in $F_{25 \% S S B}$, while $L m a t$ and $K$ did not have a significant effect $(\mathrm{P}>0.1958)$. The predictive function of species sensitivity for Actinopterygia was estimated as:

$$
F_{25 \% S S B, A C}=\exp \left(6.99(0.28)-1.55(0.08) * \ln \left(L_{\infty}\right)\right)
$$

Values in parentheses denote standard deviation of the estimates. The parameter estimates for

284 Chondrichthyes were not significantly different from $0(\mathrm{P}=0.08)$. While taxonomy and 
asymptotic length provided significant information on sensitivity, $28 \%$ of the variation was not predictable using linear relationships with taxonomy, $L_{\infty}, K$ and $L_{\text {mat }}$ and hence, the full model should be used whenever possible. The limit between low and medium productivity (high sensitivity) fish given in Hobday et al. (2011) for maximum length ( $>300 \mathrm{~cm}$ and 100 $300 \mathrm{~cm}$, respectively) and length at maturity (>200 $\mathrm{cm}$ and 40-200 cm, respectively) were insufficient to describe the current sensitive species, as 24 sensitive species had values of asymptotic length $<100 \mathrm{~cm}$ and six additional species had maximum length $<100 \mathrm{~cm}$. Five species had a length at maturity $<40 \mathrm{~cm}$.

Among the 59 sensitive taxa, landings of four species (6.8\%) were subject to species-specific quota management for defined management units, and a further five taxa $(8.5 \%)$ by genus or family-based quota management (Table S5). Additionally, 13 species of skate (22.0\%) would be included within quotas largely set for the complex (Rajiformes). Whilst $13(22.0 \%)$ of the taxa were subject to conservative management (e.g. a combination of prohibited listings and near-zero bycatch TACs), the remaining 24 species (40.7\%) are not currently subject to any management measures.

Five high value species had landing values per kg greater than cod (Dicentrarchus labrax, Hippoglossus hippoglossus, Lepidorhombus whiffiagonis, Scophthalmus rhombus, Lophius budegassa and L. piscatorius). Another 10 medium value species attained values between that of cod and half this value (Anarhichas lupus, Etmopterus princeps, Macrourus berglax,

307 Molva dypterygia, Molva macropthalma, Molva molva, Scyliorhinus canicula, Scyliorhinus stellaris, Sebastes spp. and Squalus acanthias). The remaining species were categorized as

309 low value species. 
312 Of the taxa classified as sensitive, 31 were recorded with sufficient frequency and accuracy in

313 the surveys to allow estimation of trends (Table 2). For some species, indices were highly

314 correlated, whereas for others, the surveys differed in development (Figure 3). The

315 abundance of 24 of the 31 species increased from 1980-2018 (Table 2, Figures 3-4). Among

316 the species showing significant trends, 15 increased and three (starry ray Amblyraja radiata,

317 wolf-fish Anarhichas lupus and tusk Brosme brosme) decreased. These three are at their

318 southern boundary in the surveyed area (Figure 2) whereas this was not the case for the

319 remaining species (except halibut Hippoglossus hippoglossus; Figure S1). Starry ray and tusk

320 decreased at a significantly greater rate in the later period than in the period as a whole

$321 \quad(\mathrm{P}<0.0181$ in both cases $)$. Among the 10 species showing a significant change in the trend

322 between 1980-1999 and 2000-2018, six exhibited an increasing trend while four

323 deteriorated. Fourteen species showed no significant change between time periods while the

324 remaining six species had insufficient historical data to test for changes in trends.

325 Categorising the surveys individually according to the trend estimated, trends in 42 of 78

326 combinations of species and surveys from 1980-1999 were positive, whereas the

327 corresponding number after 2000 was 161 of 209 , a change from $54 \%$ to $77 \%$.

328 Among the species listed by IUCN as Critically Endangered, Endangered or Vulnerable, four

329 taxa increased significantly after 2000 (Dipturus spp., Leucoraja circularis, Mustelus spp.

330 and Squalus acanthias) whereas four showed no significant change (Dasyatis pastinaca,

331 Galeorhinus galeus, Leucoraja fullonica and Hippoglossus hippoglossus). Among the species

332 assessed as Least Concern, starry ray, tusk and marbled electric ray Torpedo marmorata all 333 decreased significantly from 1980 to 2018. 
334 There was no relationship between sensitivity, the presence of a catch limit, the value at

335 landing and the population index trend of the species $(\mathrm{P}>0.29)$. For example, the two least

336 sensitive of the species (starry ray and wolf-fish) declined whereas the most sensitive (the

337 common skate complex) increased. The three species showing the greatest increase in the

338 past 20 years had very different management measures: the common skate complex (to be

339 returned to the sea unharmed), Smoothhounds (no management) and undulate ray Raja

340 undulata (individual species TAC). Starry ray and wolf-fish showed comparable declines,

341 despite the former being required to be returned to the sea unharmed (since 2014) whereas

342 the latter can be landed. High and medium value species included both declining/stable

343 species (wolf-fish and halibut) and increasing species (Lophius budegassa and Squalus

344 acanthias), and the same was true of low value species (starry ray and tusk declining and 345 smoothhounds and the common skate complex increasing).

\section{Discussion}

348 Using life history parameters and knowledge of fish shape and habitat to identify the likely sensitivity for each species, we demonstrated that in general, sensitive demersal marine fish increased in abundance in the Northeast Atlantic since 2000 and in many cases had been doing so since 1980 . The analysis identified potentially sensitive species along the Northeast

352 Atlantic shelf, and the trawl survey data integration method allowed the temporal changes in species abundance to be monitored for more than half of these sensitive species. The increase in abundance of most of the sensitive species analysed did not appear to be due to speciesspecific management measures, as less than half of the species were covered by catch limits and there was no strong evidence of an increase in relative abundance as fishing effort decreased after year 2000. Further, neither sensitivity nor market value appeared to be related to trends in abundance. However, the three species which declined significantly were all at 
their southern limit in the North Sea, indicating that conditions in the North Sea may be

360 affecting these species more than others, possibly due to the greater changes in water

361 temperature (Hobday and Pecl, 2014).

363 The accuracy of the identification of sensitive species depends on the accuracy of the

364 parameters included and the method used to derive sensitivity. Thorson et al. (2014)

365 demonstrated the importance of a proper literature review to derive the best available life

366 history parameter estimates. While we performed a detailed literature review, our focus was

367 on the development of the species across all of the sampled parts of the NE Atlantic, and

368 therefore we did not use local values of life history parameters. If the analysis is repeated for

369 a specific area, local values could be used instead, but as information is often sparse for

370 species identified as sensitive, using local values increases the risk of basing the analyses on

371 very limited data, and hence, it may be preferable to use the values given here for analyses

372 even at a more limited spatial scale. While our results on species sensitivity ranking are in

373 broad agreement with findings from other studies, in that larger, slower growing, late

374 maturing species are the most vulnerable to fisheries generated mortality (e.g. Jennings et al.,

375 1999; Ravard et al., 2014), sensitivities based on the life history model were not well

376 explained by a linear combination of life history parameters causing our results to differ from

377 those of Greenstreet et al. (2012). Our results also differed from those of Le Quesne and

378 Jennings (2012) perhaps as a result of including group-specific catchabilities. The methods

379 used to derive catch efficiencies assumed all individuals within a certain size and habitat

380 group are equally likely to be caught in the trawl (Walker et al., 2017). This assumption may

381 not be appropriate for species with variable behavioural responses to the trawl and species

382 that occur on non-trawlable or deeper grounds, such as rocky bottoms, reefs and wrecks. For

383 example, conger eel Conger conger inhabits rocky areas of the continental shelf and shelf 
slope (Xavier et al., 2010). Once settled, it has a relatively sedentary lifestyle. Walker et al. (2017) assumed conger eel to be caught with the same catch efficiency at size as other eellike species encountered near the seabed, which may be an overestimate. Similar considerations may apply to other species favouring untrawlable habitats. Furthermore, our study did not consider sensitivity of the species to pelagic fisheries or earlier targeted

389 fisheries. Species like basking shark and thresher shark would owe their current IUCN

390 Endangered status to these factors, rather than demersal fisheries.

391 Information about the survival of individuals that are discarded from different gear types was 392 not available. However, many bycatch species are discarded and, if they survive, their 393 populations may be more resilient to fishing than their life history parameters would indicate.

394 For most fish species, discard mortality will increase with the duration and depth of the tow, the time spent on deck and handling/discarding practices, but survival can also vary in response to size, sex, temperature, catch composition and handling (Ellis et al., 2017). Recent work on capture-release mortality could be used to improve the knowledge of the degree to which released fish survive to reenter the population (Dapp et al., 2016). Needless to say, discard survival will not impact species which are landed.

400

Spatially explicit models and management measures are potentially useful alternatives for managing sensitive species (Duggan et al., 2015; Walker et al., 2019). Spatially explicit models may provide indication of whether some species are likely to be at greater risk due to

404 fishing than expected from our analyses and whether catches of sensitive species observed to 405 be in decline can be limited by spatial measures (Shephard et al., 2012). However, such analyses must be based on a reliable distribution of the sensitive species, and this was only available for about half the sensitive species identified here. 
409 Le Quesne and Jennings (2012) suggested that significant reductions of fishing effort in the

410 Celtic Sea were required to allow sensitive species such as the common skate complex,

411 spurdog and spotted ray Raja montagui to increase. The same conclusion was reached for the

412 North Sea by Walker et al. (2019) for wolf-fish, anglerfish, brill, starry ray, spurdog,

413 smoothhounds, spotted ray and thornback ray (excluding the species not listed here as

414 sensitive). Among these eight species, five were observed as increasing in this study. This

415 may indicate either that these species are less frequently caught outside the heavily fished

416 North and Celtic Sea, providing the possibility for them to increase in less fished areas, or

417 that they are experiencing an increase in productivity due to, for example, warming waters or

418 other ecosystem changes.

419

420 The IUCN ratings of the species were in broad agreement with the sensitivity of the species,

421 and species ranked in the Critically Endangered, Endangered or Vulnerable categories were

422 generally classified as sensitive in our analysis. However, the IUCN ranking of different

423 species was not a reliable indicator of the potential for the species to sustain fishing pressure

424 - the Least Concern and Data Deficient species included starry ray, tusk, marbled electric ray

425 and wolf-fish, which were all ranked as sensitive and all decreased significantly from 1980

426 to 2018 , indicating the potential error of assuming that species listed in these categories are

427 not under pressure. Ideally, the status of these species should be updated during the next

428 IUCN assessment cycle using survey data under the IUCN criteria (IUCN Standards and

429 Petition Subcommittee, 2017). On the positive side, just above half of the species listed by

430 IUCN as Critically Endangered, Endangered or Vulnerable increased significantly since

4312000 , hence providing some confidence that these species may in time move out of these

432 categories. 
434 The lack of a direct management of catches of a large proportion of the sensitive species

435 means that some of the species in decline can continue to be caught. We stress here, that

436 while the assumption that the fishing mortality affecting non-target species is unlikely to

437 exceed the fishing mortality affecting target species (Pope et al. 2000), is likely to hold for

438 many species, this is not necessarily the case for species with restricted distributions. Further,

439 the fishing mortality predicted to generate MSY for the more productive species may not be

440 sustainable for sensitive species. Species experiencing negative effects of other pressures or

441 drivers (e.g. climate change) may also be more sensitive to fishing mortality than their

442 general life history parameters would suggest. Due to these caveats, species like wolf-fish,

443 tusk and starry ray, which are at the southern limit of their distributions in the North Sea, are

444 likely to be more sensitive to fishing than indicated in our sensitivity ranking. We

445 recommend further monitoring of sensitive species and more regular assessments of their

446 status to spur management actions before species enter the Critically Endangered,

447 Endangered or Vulnerable IUCN categories. Further, we recommend taking additional

448 precaution where species are under combined pressure from climate change and fishing.

\section{Authors' contributions}

452 HG and AR conceived the ideas and designed the analyses; HG conducted the literature 453 review and estimated species sensitivity, AR analysed species abundance and led writing of 454 the manuscript. All authors contributed critically to the development of the analyses and 455 manuscript and approved the final draft. 
458 AR \& DR received funding from the PROBYFISH project (EASME/EMFF/2017/022). DR

459 also received funding from the Department of Agriculture, Food and the Marine's

460 Competitive Research Funding Programmes. The funding bodies accept no responsibility for 461 the paper.

\section{Data availability statement}

463 The data used in this work are life history parameters as given in Tables S2 and S3 in the 464 Supporting Information and are available in Dryad as Rindorf (2020) "Life history traits and 465 abundance of Northeast Atlantic fish" (doi:10.5061/dryad.8sf7m0cjd).

466 7. References

467 Beissinger, S.R., \& McCullough, D.R. (2002). Population viability analysis. Chicago: $468 \quad$ University of Chicago Press.

469 Beverton, R.J., \& Holt, S.J. (1957). On the dynamics of exploited fish populations, Fishery $470 \quad$ Investigations Series II, Vol. XIX, Ministry of Agriculture. Fisheries and Food, 1, 957.

471 Brooks, E.N., Powers, J.E., \& Cortés, E. (2010). Analytical reference points for age472 structured models: application to data-poor fisheries. ICES Journal of Marine Science, $473 \quad 67(1), 165-175$.

474 Catchpole, T.L., Ribeiro-Santos, A., Mangi, S.C., Hedley, C., \& Gray, T.S. (2017). The 475 challenges of the landing obligation in EU fisheries. Marine Policy, 82, 76-86.

476 CBD (2014). Ecosystem approach sourcebook. Retrieved October $1^{\text {st }} 2019$ from 477 https://www.cbd.int/ecosystem/sourcebook/default.shtml.

478 Charnov, E.L., Gislason, H., \& Pope, J.G. (2013). Evolutionary assembly rules for fish life 479 histories. Fish and Fisheries, 14(2), 213-224. 
Dapp, D.R., Walker, T.I., Huveneers, C., \& Reina, R.D. (2016). Respiratory mode and gear type are important determinants of elasmobranch immediate and post-release mortality. Fish and Fisheries, 17(2), 507-524.

Duggan, J.M., Eichelberger, B.A., Ma, S., Lawler, J.J., \& Ziv, G. (2015). Informing management of rare species with an approach combining scenario modeling and spatially explicit risk assessment. Ecosystem Health and Sustainability, 1(6), 1-18.

Ellis, J.R., McCully Phillips, S. R., \& Poisson, F. (2017). A review of capture and postrelease mortality of elasmobranchs. Journal of Fish Biology, 90(3), 653-722.

European Commission. (2008). Directive 2008/56/EC of the European Parliament and of the Council of 17 June 2008 establishing a framework for community action in the field of marine environmental policy (Marine Strategy Framework Directive). http://data.europa.eu/eli/dir/2008/56/oj

European Commission. (2018a). Council Regulation (EU) 2018/120 of 23 January 2018 fixing for 2018 the fishing opportunities for certain fish stocks and groups of fish stocks, applicable in Union waters and, for Union fishing vessels, in certain non-Union waters, and amending Regulation (EU).

European Commission. (2018b). Council Regulation (EU) 2018/2025 of 17 December 2018 fixing for 2019 and 2020 the fishing opportunities for Union fishing vessels for certain deep-sea fish stocks

Fernandes, P.G., Ralph, G.M., Nieto, A., et al. (2017). Coherent assessments of Europe's marine fishes show regional divergence and megafauna loss. Nature Ecology \& Evolution, 1, p.0200.

Froese, R. and Binohlan, C., (2000). Empirical relationships to estimate asymptotic length, length at first maturity and length at maximum yield per recruit in fishes, with a simple method to evaluate length frequency data. Journal of Fish Biology, 56(4), 758-773. 
505 García-Carreras, B., Jennings, S., \& Le Quesne, W.J. (2015). Predicting reference points and 506 associated uncertainty from life histories for risk and status assessment. ICES Journal of $507 \quad$ Marine Science, 73(2), 483-493.

508 Gislason, H., Pope, J.G., Rice, J.C., \& Daan, N. (2008). Coexistence in North Sea fish 509 communities: implications for growth and natural mortality. ICES Journal of Marine $510 \quad$ Science, 65(4), 514-530.

511 Greenstreet, S.P., Rossberg, A.G., Fox, C.J., Le Quesne, W.J., Blasdale, T., Boulcott, P., 512 Mitchell, I., Millar, C. and Moffat, C. F. (2012). Demersal fish biodiversity: species-level 513 indicators and trends-based targets for the Marine Strategy Framework Directive. ICES $514 \quad$ Journal of Marine Science, 69(10), 1789-1801.

515 Hobday, A.J., \& Pecl, G.T. (2014). Identification of global marine hotspots: sentinels for 516 change and vanguards for adaptation action. Reviews in Fish Biology and Fisheries, 24(2), $517 \quad 415-425$.

518 Hobday, A.J., Smith, A.D.M., Stobutzki, I.C., et al. (2011). Ecological risk assessment for the 519 effects of fishing. Fisheries Research, 108(2-3), 372-384.

520 ICES. (2018a). Baltic Sea Ecoregion - Fisheries overview.

$521 \quad$ https://doi.org/10.17895/ices.pub.4389

522 ICES. (2018b). Greater North Sea Ecoregion - Fisheries overview.

523 https://doi.org/10.17895/ices.pub.4647

524 ICES. (2018c). Celtic Seas Ecoregion - Fisheries overview.

$525 \quad$ https://doi.org/10.17895/ices.pub.4640

526 IUCN Standards and Petition Subcommittee (2017).). Guidelines for Using the IUCN Red 527 List Categories and Criteria. Version 13. Prepared by the Standards and Petitions 528 Subcommittee. Available at http://www.iucnredlist.org/documents/RedListGuidelines.pdf 
529 Jennings, S., Greenstreet, S., \& Reynolds, J. (1999). Structural change in an exploited fish 530 community: a consequence of differential fishing effects on species with contrasting life 531 histories. Journal of Animal Ecology, 68(3), 617-627.

532 Le Quesne, W.J., \& Jennings, S. (2012). Predicting species vulnerability with minimal data to 533 support rapid risk assessment of fishing impacts on biodiversity. Journal of Applied 534 Ecology, 49(1), 1-20.

535 Nieto, A., Ralph, G.M., Comeros-Raynal, M.T., et al. (2015). European Red List of marine 536 fishes. Luxembourg: Publications Office of the European Union.

537 Pope, J.G., Macdonald, D.S., Daan, N., Reynolds, J.D. \& Jennings, S. (2000) Gauging the 538 impact of fishing mortality on non-target species. ICES Journal of Marine Science, 57, $539 \quad 689-696$.

540 Ravard, D., Brind'Amour, A., \& Trenkel, V.M. (2014). Evaluating the potential impact of 541 fishing on demersal species in the Bay of Biscay using simulations and survey data. $542 \quad$ Fisheries Research, 157, 86-95.

543 Reid, D.G., Allen, V.J., Bova, D.J., Jones, E.G., Kynoch, R.J., Peach, K.J., Fernandes, P.G. \& 544 Turrell, W.R. (2007). Anglerfish catchability for swept-area abundance estimates in a new $545 \quad$ survey trawl. ICES Journal of Marine Science, 64(8), 1503-1511.

546 Reynolds, J. D., Dulvy, N. K., Goodwin, N. B., \& Hutchings, J. A. (2005). Biology of 547 extinction risk in marine fishes. Proceedings of the Royal Society of London B: Biological $548 \quad$ Sciences, 272(1579), 2337-2344.

549 Rindorf, A. (2020), Life history traits and abundance of Northeast Atlantic fish, v2, Dryad, $550 \quad$ Dataset, https://doi.org/10.5061/dryad.8sf7m0cjd

551 Rindorf, A., Mumford, J., Baranowski, P., Clausen, L.W., García, D., Hintzen, N.T., Kempf, 552 A., Leach, A., Levontin, P., Mace, P., Mackinson, S., Maravelias, C., Prellezo, R., 

to reflect multidimensional fisheries management objectives. Marine Policy, 85, 33-41.

Shephard, S., Gerritsen, H., Kaiser, M.J., Reid, D.G. (2012). Spatial heterogeneity in fishing 556 creates de facto refugia for Endangered Celtic Sea elasmobranchs. PLoS ONE, 7(11).

557 Walker, N.D., Maxwell, D.L, Le Quesne, W.J.F., \& Jennings, S. (2017). Estimating

558 efficiency of survey and commercial trawl gears from comparisons of catch-ratios. ICES $559 \quad$ Journal of Marine Science, 74(5), 1448-1457.

560 Walker, N.D., García-Carreras, B., Le Quesne, W.J., Maxwell, D.L., \& Jennings, S. (2019). 561 A data-limited approach for estimating fishing mortality rates and exploitation status of 562 diverse target and non-target fish species impacted by mixed multispecies fisheries. ICES 563 Journal of Marine Science, 76, 824-836.

564 Xavier, J.C., Cherel, Y., Assis, C.A., Sendão, J., \& Borges, T.C. (2010). Feeding ecology of 565 conger eels (Conger conger) in north-east Atlantic waters. Journal of the Marine 566 Biological Association of the United Kingdom, 90(3), 493-501. 
TABLE 1. Life history model equations.

\begin{tabular}{|c|c|c|c|}
\hline \multicolumn{2}{|c|}{ Life history component } & Equation & Reference/Assumption/Data \\
\hline \multicolumn{4}{|c|}{ Deterministic relationships } \\
\hline \multicolumn{2}{|c|}{ Asymptotic length, $\boldsymbol{L}_{\infty}(\mathrm{cm})$} & $L_{\infty}=10^{\left(0.044+0.9841 \log _{10}\left(L_{\max }\right)\right)}$ & Froese \& Binohlan (2000) \\
\hline \multicolumn{2}{|c|}{$\begin{array}{c}\text { Von Bertalanffy parameter, } \boldsymbol{K} \\
\left(\text { year }^{-1}\right)\end{array}$} & $K=1.447 * L_{\infty}^{-0.5132}$ & Data from Table S2 \\
\hline \multicolumn{2}{|c|}{ Length at first maturity, $\boldsymbol{L}_{\text {mat }}$} & $L_{\text {mat }}=0.6106 * L_{\infty}^{0.9684}$ & Data from Table S2 \\
\hline \multirow{3}{*}{$\begin{array}{l}\text { Minimum } \\
\text { length, } \\
\boldsymbol{L}_{\min }(\mathrm{cm})\end{array}$} & Egg-laying Elasmob. & $L_{\min }=0.7918 * L_{\infty}^{0.5921}$ & Data from Table S2 \\
\hline & $\begin{array}{c}\text { Live-bearing } \\
\text { Elasmob. }\end{array}$ & $L_{\min }=0.5399 L_{\infty}^{0.7977}$ & Data from Table S2 \\
\hline & Actinopterygians & $L_{\min }=2$ & Assumed \\
\hline \multicolumn{2}{|c|}{ Body weight at length $L, W_{L}(\mathrm{~g})$} & $W_{L}=0.01 * L^{3}$ & Gislason et al. (2008) \\
\hline \multicolumn{4}{|c|}{ Model equations } \\
\hline \multicolumn{2}{|c|}{ Proportion mature at length, $\boldsymbol{P}_{\boldsymbol{L}}$} & $P_{L}=\left(1+e^{\left(5.5 *\left(1-L / L_{m a t}\right)\right.}\right)^{-1}$ & Assuming L $75 \%=1.2 * L_{\text {mat }}$ \\
\hline \multicolumn{2}{|r|}{$M_{L}\left(\mathrm{y}^{-1}\right)$} & $M_{L}=K *\left({ }^{L_{t}} /_{L_{\infty}}\right)^{-1.5}$ & Charnov et al. (2013) \\
\hline Fishing mc & $\begin{array}{l}\text { tality at length, } \boldsymbol{F}_{\boldsymbol{L}}\left(\mathrm{y}^{-}\right. \\
\text {1) }\end{array}$ & $F_{L}=F_{\text {factor }} *$ Catch eff. $(L)$ & Walker et al. (2017) \\
\hline \multicolumn{2}{|c|}{ Total mortality, $\boldsymbol{Z}_{\boldsymbol{L}}\left(\mathrm{y}^{-1}\right)$} & $Z_{L+\Delta L / 2}=F_{L+\Delta L / 2}+M_{L+\Delta L / 2}$ & \\
\hline \multicolumn{2}{|c|}{ Numbers at length $L+\Delta L$} & $\begin{array}{l}N_{L+\Delta L} \\
=N_{L}\left(\frac{L_{\infty}-(L+\Delta L)}{L_{\infty}-L}\right)^{Z_{L} / K}\end{array}$ & Gislason et al. (2008) \\
\hline
\end{tabular}




\begin{tabular}{|c|l|l|}
\hline $\begin{array}{c}\text { Average numbers in length } \\
\text { interval }\end{array}$ & $\bar{N}_{L, L+\Delta L}=\frac{N_{L}-N_{L+\Delta L}}{Z_{L+\Delta L / 2}}$ & Gislason et al. (2008) \\
\hline Spawning stock per recruit & $\frac{S S B}{R}=\sum_{L=L_{\text {min }}}^{L_{\infty}} \frac{P_{L} \bar{N}_{L, L+\Delta L} W_{L+\Delta L / 2}}{N_{L_{\text {min }}}}$ & Gislason et al. (2008) \\
\hline
\end{tabular}

570

571 
573 TABLE 2. Annual change in species $\ln$ (index) for the periods 1980-2018 and 2000-2018.

\begin{tabular}{|c|c|c|c|c|c|}
\hline \multirow[t]{9}{*}{ Species } & Annual change & Annual change & Probability & Number & Number \\
\hline & from 1980 to & from 2000 to & of same & of & of \\
\hline & 2018 & 2018 & trend from & surveys & surveys \\
\hline & & & $1980-$ & with & with \\
\hline & & & 1999 and & positive & positive \\
\hline & & & $2000-$ & trend & trend \\
\hline & & & 2018 & $1980-$ & $2000-$ \\
\hline & & & & 1999/all & 2018/all \\
\hline & & & & surveys & surveys \\
\hline Amblyraja & $-0.032(0.008$ & $-0.069(0.009$ & 0.0181 & $1 / 2$ & $2 / 5$ \\
\hline radiata & $P=0.0002)$ & $\mathrm{P}<\mathbf{0 . 0 0 0 1 )}$ & & & \\
\hline \multirow[t]{2}{*}{ Anarhichas lupus } & $\mathbf{- 0 . 0 7 0}(\mathbf{0 . 0 0 7}$ & $-0.081(0.022$ & 0.7383 & $0 / 2$ & $1 / 4$ \\
\hline & $\mathrm{P}<0.0001)$ & $P=0.0019)$ & & & \\
\hline \multirow[t]{2}{*}{ Brosme brosme } & $-0.025(0.007$ & $-0.078(0.015$ & 0.0042 & $2 / 3$ & $2 / 5$ \\
\hline & $P=0.0010)$ & $\mathrm{P}<0.0001)$ & & & \\
\hline Chimaera & $0.045(0.017$ & $0.067(0.015$ & 0.1080 & $2 / 2$ & $4 / 5$ \\
\hline monstrosa & $P=0.0152)$ & $P=0.0003)$ & & & \\
\hline \multirow[t]{2}{*}{ Conger conger } & $0.052(0.008$ & $0.000(0.008$ & 0.0786 & $2 / 3$ & $5 / 10$ \\
\hline & $\mathbf{P}<0.0001)$ & $\mathrm{P}=0.9936)$ & & & \\
\hline Dasyatis & $-0.000(0.027$ & $0.006(0.036$ & & & $2 / 2$ \\
\hline pastinaca & $\mathrm{P}=0.9972)$ & $\mathrm{P}=0.8714)$ & & & \\
\hline Dipturus batis & $0.075(0.012$ & $0.092(0.009$ & 0.5196 & $4 / 4$ & $5 / 6$ \\
\hline complex & $\mathrm{P}<0.0001)$ & $\mathrm{P}<0.0001)$ & & & \\
\hline
\end{tabular}




\begin{tabular}{|c|c|c|c|c|c|}
\hline $\begin{array}{l}\text { Galeorhinus } \\
\text { galeus }\end{array}$ & $\begin{array}{l}-0.011(0.019 \\
\mathrm{P}=0.5632)\end{array}$ & $\begin{array}{l}0.039(0.034 \\
\mathrm{P}=0.2759)\end{array}$ & 0.6018 & $2 / 3$ & $4 / 4$ \\
\hline Galeus & $0.065(0.012$ & 0.064 (0.011, & 0.5192 & $2 / 4$ & $8 / 8$ \\
\hline melastomus & $\mathrm{P}<0.0001)$ & $\mathrm{P}<0.0001)$ & & & \\
\hline Hexanchus & $0.028(0.015$ & $0.028(0.015$ & & & $1 / 1$ \\
\hline griseus & $\mathrm{P}=0.0890)$ & $\mathrm{P}=0.0890)$ & & & \\
\hline Hippoglossus & $0.012(0.009$ & $-0.029(0.027$ & 0.4556 & $1 / 2$ & $0 / 2$ \\
\hline hippoglossus & $\mathrm{P}=0.2041)$ & $\mathrm{P}=0.2940)$ & & & \\
\hline Lepidorhombus & $0.023(0.004$ & $0.009(0.008$ & 0.4411 & $3 / 5$ & $14 / 14$ \\
\hline whiffiagonis & $P<0.0001)$ & $\mathrm{P}=0.2674)$ & & & \\
\hline Leucoraja & $0.035(0.020$ & 0.074 (0.017, & & & $3 / 4$ \\
\hline circularis & $\mathrm{P}=0.0959)$ & $P=0.0006)$ & & & \\
\hline Leucoraja & $-0.008(0.013$ & $0.023(0.018$ & 0.9217 & $1 / 1$ & $2 / 3$ \\
\hline fullonica & $\mathrm{P}=0.5550)$ & $\mathrm{P}=0.2077)$ & & & \\
\hline \multirow[t]{2}{*}{ Leucoraja naevus } & $0.030(0.006$ & $0.001(0.007$ & 0.0551 & $4 / 5$ & $8 / 10$ \\
\hline & $\mathrm{P}<0.0001)$ & $\mathrm{P}=0.9004)$ & & & \\
\hline Lophius & $0.028(0.009$ & $0.048(0.008$ & 0.0410 & $1 / 3$ & $7 / 9$ \\
\hline budegassa & $P<0.0053)$ & $\mathrm{P}<0.0001)$ & & & \\
\hline Lophius & $0.026(0.008$ & $0.002(0.010$ & 0.0014 & $3 / 5$ & $6 / 12$ \\
\hline piscatorius & $P=0.0046)$ & $\mathrm{P}=0.8562)$ & & & \\
\hline Molva & $0.122(0.039$ & $0.054(0.037$ & & & $3 / 3$ \\
\hline macropthalma & $P=0.0054)$ & $\mathrm{P}=0.1597)$ & & & \\
\hline
\end{tabular}




\begin{tabular}{|c|c|c|c|c|c|}
\hline Molva molva & $\begin{array}{l}0.016(0.007 \\
P=0.0213)\end{array}$ & $\begin{array}{l}0.049(0.012 \\
P=0.0007)\end{array}$ & 0.0146 & $1 / 4$ & $5 / 8$ \\
\hline Mustelus spp. & $\begin{array}{l}0.095(0.010 \\
P<0.0001)\end{array}$ & $\begin{array}{l}0.122(0.010 \\
P<0.0001)\end{array}$ & 0.2205 & $3 / 4$ & $8 / 8$ \\
\hline $\begin{array}{l}\text { Phycis } \\
\text { blennoides }\end{array}$ & $\begin{array}{l}0.059(0.015 \\
P=0.0006)\end{array}$ & $\begin{array}{l}-0.017(0.018 \\
\mathrm{P}=0.3785)\end{array}$ & 0.0010 & $2 / 3$ & $4 / 7$ \\
\hline Raja brachyura & $\begin{array}{l}0.011(0.014 \\
\mathrm{P}=0.4289)\end{array}$ & $\begin{array}{l}0.059(0.012 \\
P<0.0001)\end{array}$ & 0.0005 & $0 / 4$ & $6 / 7$ \\
\hline Raja clavata & $\begin{array}{l}0.016(0.008 \\
P=0.0656)\end{array}$ & $\begin{array}{l}\text { 0.086 (0.007, } \\
\mathrm{P}<0.0001)\end{array}$ & $<0.0001$ & $2 / 6$ & $11 / 13$ \\
\hline $\begin{array}{l}\text { Raja } \\
\text { microocellata }\end{array}$ & $\begin{array}{l}0.009(0.009 \\
\mathrm{P}=0.3126)\end{array}$ & $\begin{array}{l}0.000(0.012 \\
\mathrm{P}=0.9995)\end{array}$ & & & $1 / 3$ \\
\hline Raja montagui & $\begin{array}{l}0.054(0.009 \\
P<0.0001)\end{array}$ & $\begin{array}{l}\text { 0.050 (0.013, } \\
P=0.0015)\end{array}$ & 0.8078 & $2 / 6$ & $10 / 10$ \\
\hline Raja undulata & $\begin{array}{l}0.069(0.019 \\
P=0.0009)\end{array}$ & $\begin{array}{l}0.099(0.043 \\
P=0.0339)\end{array}$ & 0.8856 & $1 / 1$ & $3 / 3$ \\
\hline $\begin{array}{l}\text { Scophthalmus } \\
\text { rhombus }\end{array}$ & $\begin{array}{l}0.046(0.008 \\
P<0.0001)\end{array}$ & $\begin{array}{l}0.052(0.026 \\
\mathrm{P}=0.0593)\end{array}$ & 0.0955 & $3 / 5$ & $12 / 15$ \\
\hline $\begin{array}{l}\text { Scyliorhinus } \\
\text { canicula }\end{array}$ & $\begin{array}{l}0.041(0.005 \\
P<0.0001)\end{array}$ & $\begin{array}{l}0.069(0.005 \\
P<0.0001)\end{array}$ & $<0.0001$ & $4 / 6$ & $11 / 12$ \\
\hline $\begin{array}{l}\text { Scyliorhinus } \\
\text { stellaris }\end{array}$ & $\begin{array}{l}0.039(0.012 \\
P=0.0027)\end{array}$ & $\begin{array}{l}0.079(0.020 \\
P=0.0012)\end{array}$ & 0.0014 & $1 / 1$ & $4 / 4$ \\
\hline $\begin{array}{l}\text { Squalus } \\
\text { acanthias }\end{array}$ & $\begin{array}{l}0.039(0.008 \\
P<0.0001)\end{array}$ & $\begin{array}{l}0.067(0.021 \\
P=0.0060)\end{array}$ & 0.6783 & $0 / 4$ & $8 / 11$ \\
\hline
\end{tabular}


Torpedo

marmorata
-0.044 (0.016,

$P=0.0140)$
$-0.018(0.018$

$1 / 1$

574

575 
577 Figure 1. $F_{25 \% S S B}$ for each IUCN category (Critically Endangered (CR), Endangered (EN),

578 Vulnerable (VU), Near Threatened (NT), Least Concern (LC) and Data Deficient (DD)) All

579 values of $F_{25 \% S S B}>2$ are shown as 2 . Dotted line signifies the limit between sensitive and

580 non-sensitive species $\left(F_{25 \% S S B}=0.43\right)$.

581

582

583

584 
586 Figure 2. Distribution (colour) of selected sensitive species and extent of surveyed area (grey 587 rectangles). Left panel top to bottom: Increasing species - Common skate complex (Dipturus 588 spp.), ling (Molva molva), and lesser-spotted dogfish (Scyliorhinus canicula). Right panel top 589 to bottom: decreasing species - Starry ray (Amblyraja radiata), Atlantic wolf-fish

590 (Anarhichas lupus) and tusk (Brosme brosme). Distribution data are scaled to a mean of 1 in 591 the period 2009 to 2018 . The distribution of the remaining species can be found in the 592 supplementary material.

593 Figure 3. Development of species indicators for individual surveys. Each line is one survey 594 annual index.

595 Figure 4. Development of species indicators integrated across surveys and areas. Each point 596 is one survey annual index, black line is a loess fit and grey lines are the $95 \%$ confidence 597 intervals of the fitted line. Note that the points are not weighted evenly when estimating the 598 loess, hence the line is not identical to the mean of the points.

599

600 


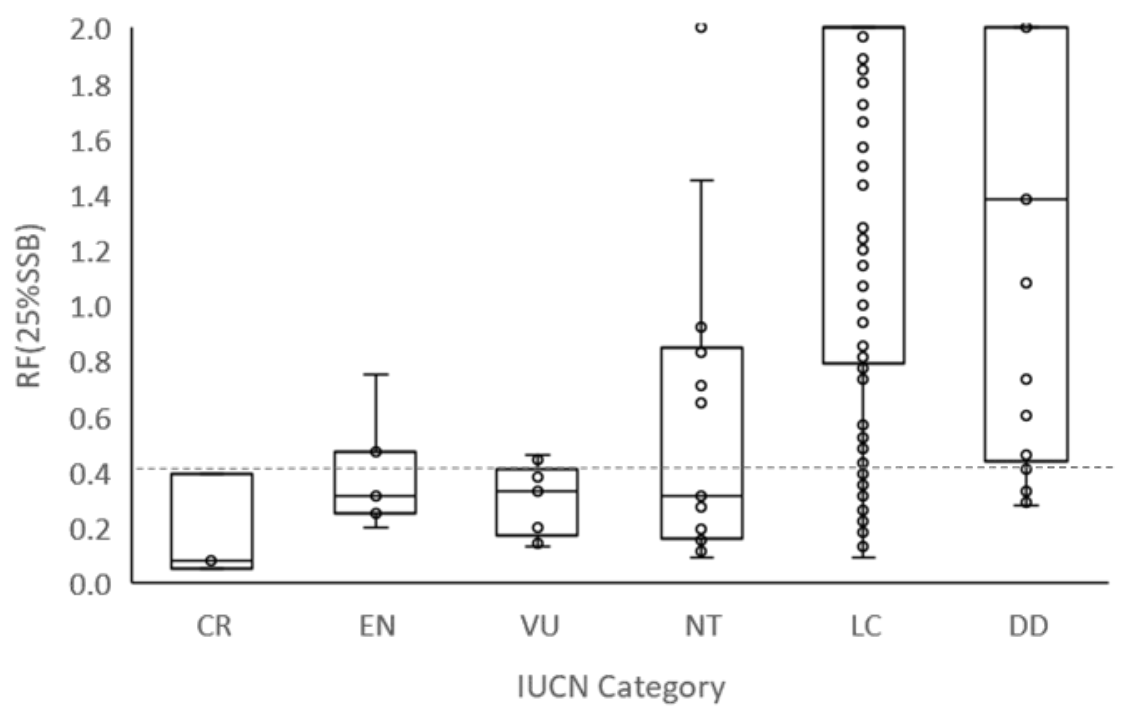




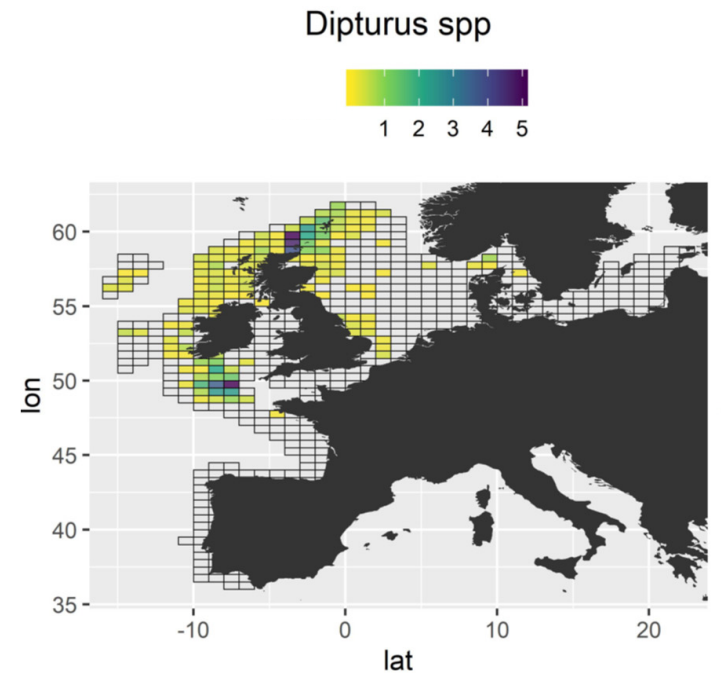

Molva molva

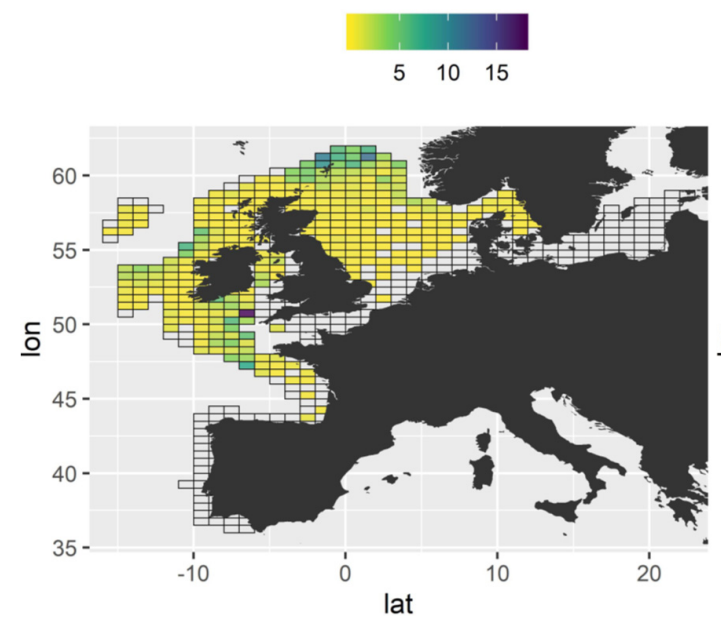

Scyliorhinus canicula
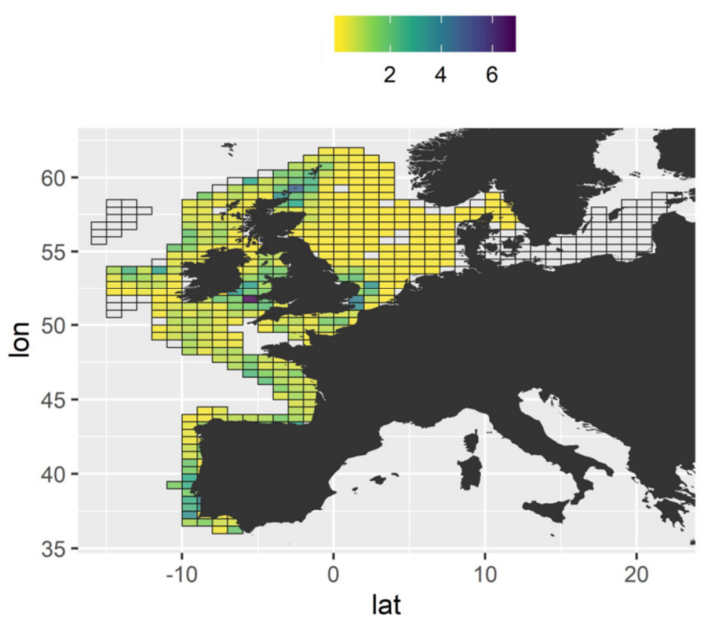

Amblyraja radiata
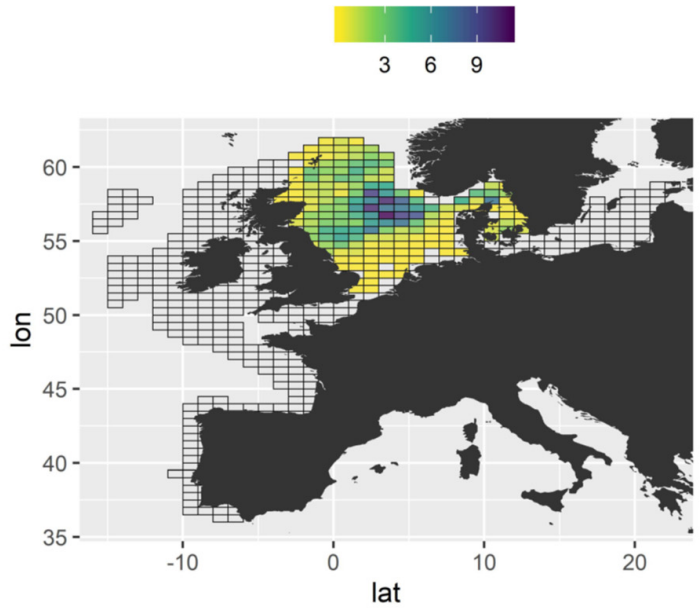

Anarhichas lupus
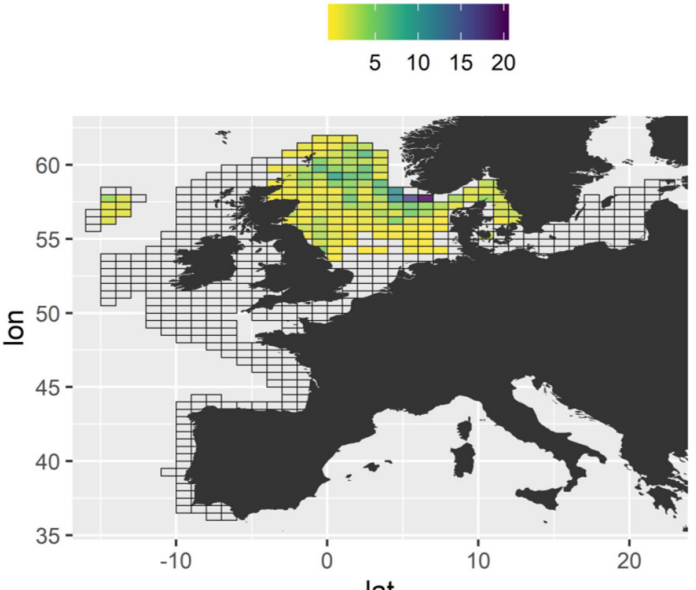

Brosme brosme
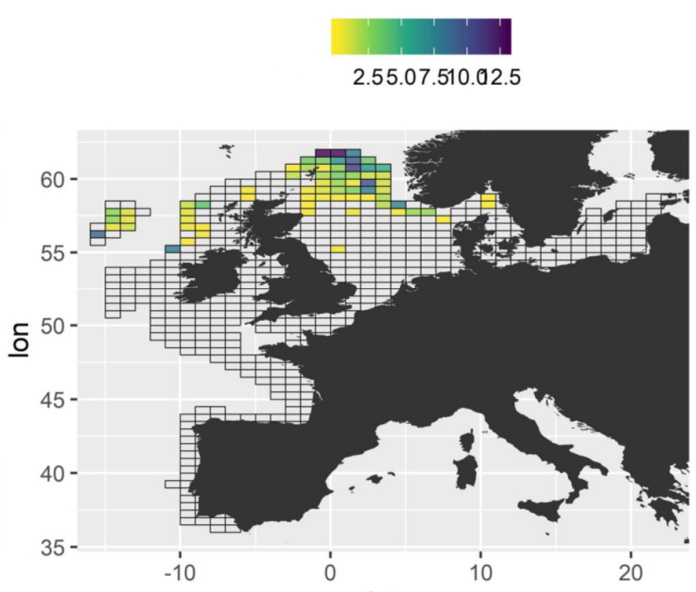

lat 


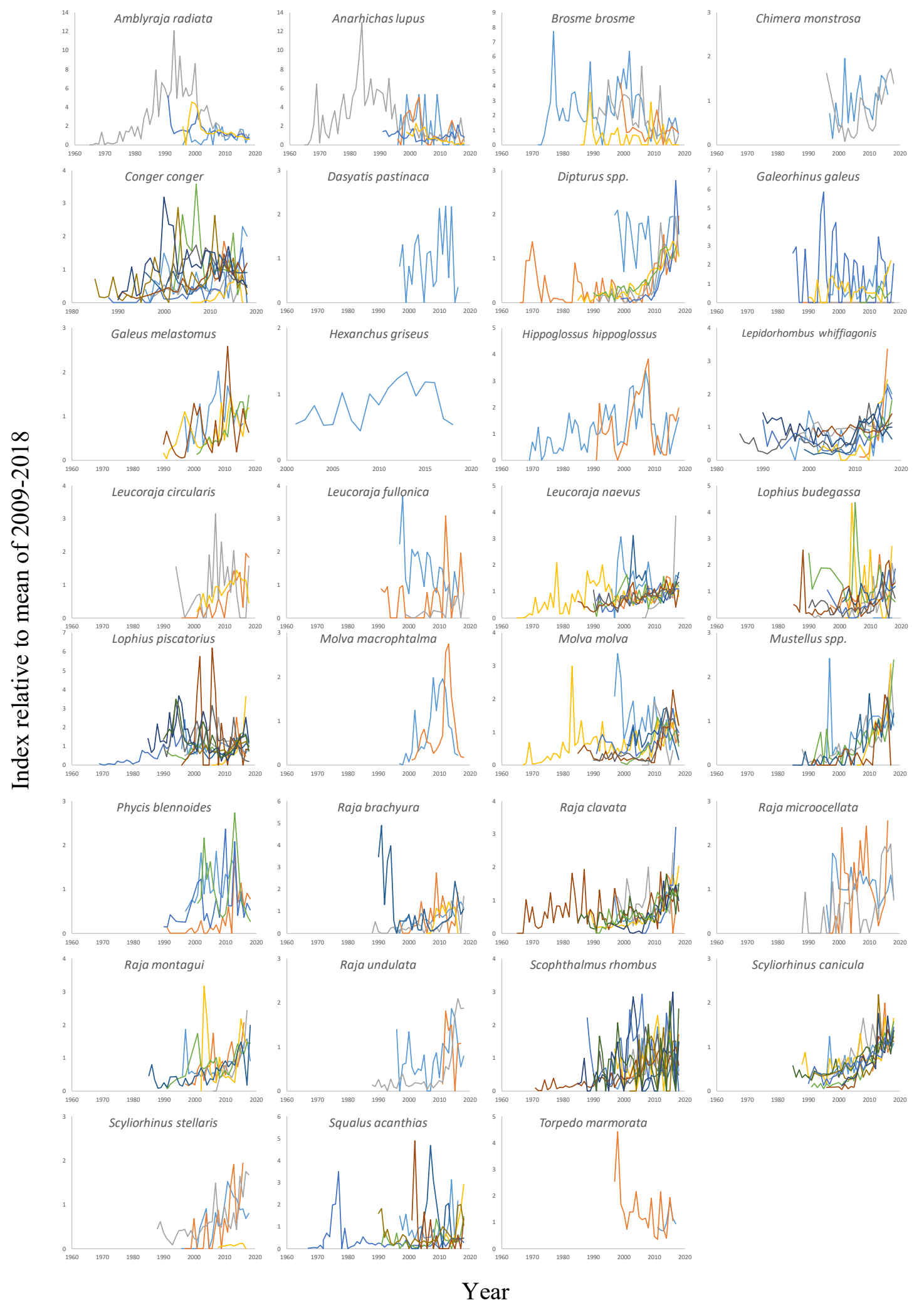




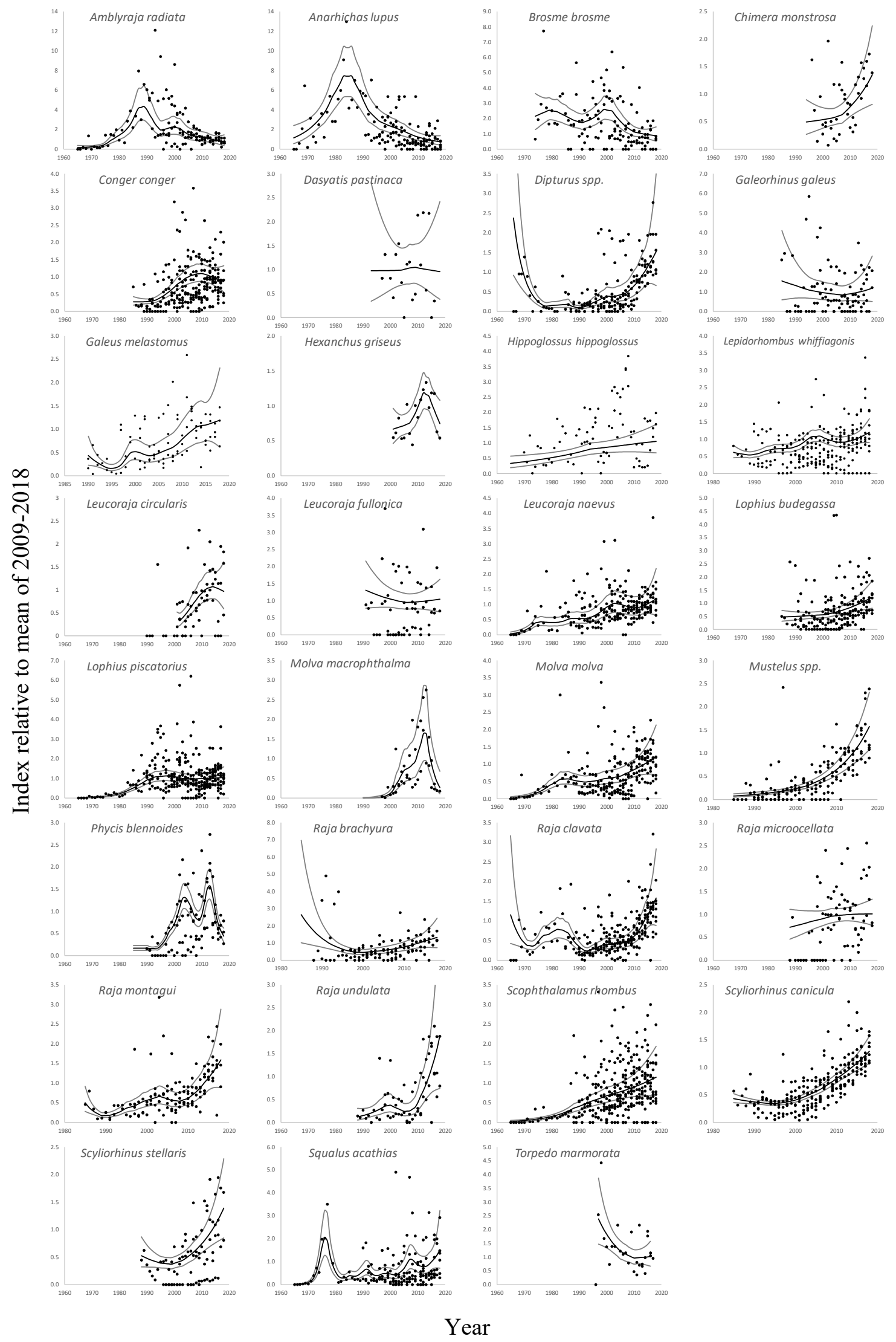




\section{Supporting Information}

Rindorf et al.: Are fish sensitive to trawling recovering in the Northeast Atlantic?

Table S1. Species groups used for calculating exploitation pattern. From Walker et al. (2017).

\begin{tabular}{ll}
\hline Species group & Group description \\
\hline $\mathbf{1}$ & Predominately buried in sediment \\
\hline $\mathbf{2}$ & On or near the seabed - eel-like \\
\hline $\mathbf{3}$ & Predominantly on the seabed - flat \\
\hline $\mathbf{5}$ & Predominantly close to the seabed, but not on it \\
\hline $\mathbf{6}$ & Midwater species with some seabed association \\
\hline $\mathbf{7}$ & Pelagic \\
\hline
\end{tabular}


Table S2. Life history parameters, type of exploitation pattern, species group and fishing mortality factor, $F_{25 \% S S B}$, necessary to reduce the spawning stock biomass, SSB, to $25 \%$ of its unfished level. For species with clear sexual dimorphism in $L_{\text {max }}, L_{\infty}$ or $K$ (e.g. Callionymus lyra), the parameters for females were used, if available.

\begin{tabular}{|c|c|c|c|c|c|c|c|c|c|c|}
\hline Species & $L_{\max }$ & $L_{\infty}$ & $K$ & $L_{\text {mat }}$ & $L_{\min }$ & $\begin{array}{l}\text { Exploitation } \\
\text { pattern }\end{array}$ & Reproduction & Taxonomy & $F_{25 \% S S B}$ & IUCN \\
\hline Rostroraja alba & & 200 & 0.04 & 129.4 & & 3 & Oviparous & Elasmobranchii & 0.05 & $C R$ \\
\hline Dipturus batis-complex & & 254 & 0.057 & 130 & 31 & 3 & Oviparous & Elasmobranchii & 0.08 & $C R$ \\
\hline Somniosus microcephalus & & 546 & & 400 & 40 & 7 & Viviparous & Elasmobranchii & 0.09 & NT \\
\hline Conger conger & & 265 & 0.07 & & 12.5 & 2 & Oviparous & Actinopterygia & 0.10 & LC \\
\hline Dipturus nidarosiensis & 200 & & & 135 & & 3 & Oviparous & Elasmobranchii & 0.11 & NT \\
\hline Dipturus oxyrinchus & & 256 & 0.04 & 83 & 17 & 4 & Oviparous & Elasmobranchii & 0.13 & NT \\
\hline Hexanchus griseus & 458 & & & 420 & 70 & 4 & Viviparous & Elasmobranchii & 0.13 & LC \\
\hline Hippoglossus hippoglossus & & 204 & 0.1 & 112 & 2 & 3 & Oviparous & Actinopterygia & 0.13 & VU \\
\hline Dasyatis pastinaca & & 120 & 0.086 & 63 & 20 & 3 & Viviparous & Elasmobranchii & 0.14 & VU \\
\hline Raja clavata & & 140 & 0.093 & 72 & 12 & 3 & Oviparous & Elasmobranchii & 0.15 & NT \\
\hline Rajella lintea & 114 & & & 97 & & 3 & Oviparous & Elasmobranchii & 0.16 & $\mathrm{LC}$ \\
\hline Lophius piscatorius & & 167 & 0.077 & 86 & 2 & 7 & Oviparous & Actinopterygia & 0.16 & LC \\
\hline Molva molva & & 183 & 0.118 & 95 & 2 & 2 & Oviparous & Actinopterygia & 0.18 & LC \\
\hline Raja brachyura & & 155 & 0.129 & 84 & 15 & 3 & Oviparous & Elasmobranchii & 0.19 & NT \\
\hline Raja microocellata & 91 & & & 78 & 13 & 3 & Oviparous & Elasmobranchii & 0.20 & NT \\
\hline Leucoraja circularis & 117 & & & & & 3 & Oviparous & Elasmobranchii & 0.20 & EN \\
\hline Leucoraja fullonica & 120 & & & & & 3 & Oviparous & Elasmobranchii & 0.20 & VU \\
\hline Molva dypterygia & & 155 & 0.126 & 88 & 2 & 2 & Oviparous & Actinopterygia & 0.20 & VU \\
\hline Raja undulata & & 113 & 0.149 & 86 & 14 & 3 & Oviparous & Elasmobranchii & 0.20 & NT \\
\hline Amblyraja hyperborea & 112 & & & & 17 & 3 & Oviparous & Elasmobranchii & 0.22 & LC \\
\hline Lophius budegassa & & 94 & 0.10 & 54 & 2 & 7 & Oviparous & Actinopterygia & 0.22 & LC \\
\hline Scyliorhinus stellaris & 162 & & & & 16 & 7 & Oviparous & Elasmobranchii & 0.22 & NT \\
\hline Deania calcea & & 119 & 0.08 & 105 & 32 & 5 & Viviparous & Elasmobranchii & 0.25 & EN \\
\hline Brosme brosme & & 84 & 0.109 & 48 & 2 & 7 & Oviparous & Actinopterygia & 0.26 & LC \\
\hline Coryphaenoides rupestris & & 76 & 0.1 & 47 & 2 & 4 & Oviparous & Actinopterygia & 0.26 & EN \\
\hline
\end{tabular}


Mora moro

Rajella bathyphila

Anarhichas minor

Raja montagui

Chimaera monstrosa

Macrourus berglax

Phycis blennoides

Brama brama

Dicentrarchus punctatus

Dasyatis tortonesei

Etmopterus princeps

Polyprion americanus

Scyliorhinus canicula

Squalus acanthias

Leucoraja naevus

Molva macrophthalma

Scophthalmus rhombus

Dalatias licha

Epigonus telescopus

Galeorhinus galeus

Mustelus mustelus/asterias

Scorpaena scrofa

Synaphobranchus kaupi

Torpedo marmorata

Lepidorhombus whiffiagonis

Lamna nasus

Rajella fyllae

Amblyraja radiata

Argyrosomus regius

\begin{tabular}{|c|c|c|c|c|}
\hline & 74 & 0.05 & & \\
\hline \multicolumn{5}{|l|}{90} \\
\hline & 107 & 0.158 & 83 & \\
\hline & 73 & 0.18 & 60 & 1 \\
\hline & 64 & 0.10 & 46 & 1 \\
\hline & 62 & 0.04 & 28.5 & \\
\hline & 113 & 0.0886 & 33 & \\
\hline & 71 & 0.08 & & \\
\hline & 103 & 0.07 & & \\
\hline 250 & & & 60 & 2 \\
\hline 75 & & & 61 & 1 \\
\hline & 152 & 0.07 & 77.9 & \\
\hline & 75 & 0.15 & 57 & \\
\hline & 121 & 0.07 & 63 & 27. \\
\hline & 84 & 0.197 & 56 & 11. \\
\hline \multicolumn{5}{|l|}{108} \\
\hline & 85 & 0.15 & 37 & \\
\hline & 165 & 0.15 & 138 & 4 \\
\hline 75 & & & 56 & \\
\hline & 163 & 0.075 & & \\
\hline & 124 & 0.146 & 85 & 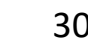 \\
\hline & 62 & 0.081 & 29 & \\
\hline \multicolumn{5}{|l|}{100} \\
\hline & 57 & 0.187 & 44 & 12 \\
\hline & 61 & 0.142 & 31 & \\
\hline & 333 & 0.061 & 245 & 7 \\
\hline \multicolumn{4}{|l|}{60} & \\
\hline & 66 & 0.23 & 46 & $\perp$ \\
\hline & 172 & 0.15 & 90 & \\
\hline
\end{tabular}

$\begin{array}{ll}5 & \text { Oviparous } \\ 3 & \text { Oviparous } \\ 7 & \text { Oviparous } \\ 3 & \text { Oviparous } \\ 4 & \text { Oviparous } \\ 5 & \text { Oviparous } \\ 7 & \text { Oviparous } \\ 4 & \text { Oviparous } \\ 5 & \text { Oviparous } \\ 4 & \text { Viviparous } \\ 4 & \text { Viviparous } \\ 5 & \text { Oviparous } \\ 7 & \text { Oviparous } \\ 5 & \text { Viviparous } \\ 3 & \text { Oviparous } \\ 4 & \text { Oviparous } \\ 3 & \text { Oviparous } \\ 4 & \text { Viviparous } \\ 4 & \text { Oviparous } \\ 5 & \text { Viviparous } \\ 4 & \text { Viviparous } \\ 7 & \text { Oviparous } \\ 4 & \text { Oviparous } \\ 3 & \text { Viviparous } \\ 3 & \text { Oviparous } \\ 6 & \text { Viviparous } \\ 3 & \text { Oviparous } \\ 3 & \text { Oviparous } \\ 4 & \text { Oviparous }\end{array}$

Actinopterygia

0.26 LC

Elasmobranchii

0.26 LC

Actinopterygia

0.27 NT

Elasmobranchii $\quad 0.27$ LC

Holocephali 0.28 NT

Actinopterygia $\quad 0.28$ LC

Actinopterygia $\quad 0.28$ DD

Actinopterygia $\quad 0.29$ LC

Actinopterygia $\quad 0.29$ LC

Elasmobranchii $\quad 0.30 \mathrm{NA}$

Elasmobranchii $\quad 0.32$ LC

Actinopterygia $\quad 0.31$ NT

Elasmobranchii 0.31 LC

Elasmobranchii $\quad 0.31$ EN

Elasmobranchii $\quad 0.32$ LC

Actinopterygia $\quad 0.34 \quad \mathrm{LC}$

Actinopterygia $\quad 0.32$ LC

Elasmobranchii $\quad 0.33$ EN

Actinopterygia $\quad 0.33$ DD

Elasmobranchii $0.33 \mathrm{VU}$

Elasmobranchii $0.33 \mathrm{VU}$

Actinopterygia $\quad 0.33$ LC

Actinopterygia $\quad 0.35$ LC

Elasmobranchii $\quad 0.34$ LC

Actinopterygia $\quad 0.35$ LC

Elasmobranchii $\quad 0.39 \mathrm{CR}$

Elasmobranchii $\quad 0.40$ LC

Elasmobranchii $\quad 0.40$ LC

Actinopterygia $\quad 0.40$ LC 


\begin{tabular}{|c|c|c|c|c|c|c|c|c|c|c|}
\hline Anarhichas lupus & & 91 & 0.23 & 73 & 2 & 7 & Oviparous & Actinopterygia & 0.41 & $\mathrm{DD}$ \\
\hline Ephippion guttifer & 80 & & & & 2 & 4 & Oviparous & Actinopterygia & 0.42 & DD \\
\hline Galeus melastomus & 67 & & & 45 & & 7 & Oviparous & Elasmobranchii & 0.44 & LC \\
\hline Torpedo nobiliana & 180 & & & & 23 & 5 & Viviparous & Elasmobranchii & 0.44 & LC \\
\hline Pollachius virens & & 107 & 0.19 & 71 & 2 & 4 & Oviparous & Actinopterygia & 0.43 & LC \\
\hline Sparus aurata & & 85 & 0.13 & 36.5 & 2 & 4 & Oviparous & Actinopterygia & 0.43 & LC \\
\hline Myliobatis aquila & 183 & & & & & 5 & Viviparous & Elasmobranchii & 0.40 & VU \\
\hline Scophthalmus maximus & & 65 & 0.26 & 46 & 2 & 3 & Oviparous & Actinopterygia & 0.46 & VU \\
\hline Solea senegalensis & & 55 & 0.18 & & 2 & 3 & Oviparous & Actinopterygia & 0.46 & $\mathrm{DD}$ \\
\hline Cetorhinus maximus & & 1000 & 0.062 & 500 & 175 & 6 & Viviparous & Elasmobranchii & 0.47 & EN \\
\hline Dicentrarchus labrax & & 85 & 0.097 & 43 & 2 & 5 & Oviparous & Actinopterygia & 0.49 & LC \\
\hline Merluccius merluccius & & 130 & 0.16 & 50 & 2 & 4 & Oviparous & Actinopterygia & 0.49 & LC \\
\hline Pollachius pollachius & & 86 & 0.186 & 47 & 2 & 4 & Oviparous & Actinopterygia & 0.49 & LC \\
\hline Nettastoma melanurum & 80 & & & 53.5 & 2 & 1 & Oviparous & Actinopterygia & 0.50 & LC \\
\hline Dentex gibbosus & & 98 & 0.15 & 34.7 & 2 & 4 & Oviparous & Actinopterygia & 0.51 & LC \\
\hline Paralichthys dentatus & & 82 & 0.24 & 37 & 2 & 3 & Oviparous & Actinopterygia & 0.52 & LC \\
\hline Lepidorhombus boscii & & 46 & 0.15 & & 2 & 3 & Oviparous & Actinopterygia & 0.53 & LC \\
\hline Trachipterus arcticus & & 303 & & & 2 & 6 & Oviparous & Actinopterygia & 0.54 & LC \\
\hline Scymnodon ringens & 110 & & & & & 5 & Viviparous & Elasmobranchii & 0.57 & LC \\
\hline Myoxocephalus quadricornis & & 62 & 0.16 & & 2 & 7 & Oviparous & Actinopterygia & 0.58 & LC \\
\hline Etmopterus pusillus & & 51 & 0.13 & 44.58 & & 4 & Viviparous & Elasmobranchii & 0.60 & $\mathrm{DD}$ \\
\hline Pagellus bogaraveo & & 51 & 0.14 & 40 & 2 & 4 & Oviparous & Actinopterygia & 0.71 & NT \\
\hline Gadus morhua & & 119 & 0.27 & 59 & 2 & 4 & Oviparous & Actinopterygia & 0.73 & LC \\
\hline Helicolenus dactylopterus & & 31 & 0.09 & 23 & 2 & 7 & Oviparous & Actinopterygia & 0.74 & LC \\
\hline Alopias vulpinus & & 484 & 0.11 & 303 & 92 & 6 & Viviparous & Elasmobranchii & 0.75 & EN \\
\hline Glyptocephalus cynoglossus & & 44 & 0.2 & & 2 & 3 & Oviparous & Actinopterygia & 0.76 & LC \\
\hline Chelon labrosus & & 61 & 0.12 & & 2 & 5 & Oviparous & Actinopterygia & 0.77 & LC \\
\hline Deania profundorum & 79 & & & & & 5 & Viviparous & Elasmobranchii & 0.79 & LC \\
\hline Gnathophis mystax & 60 & & & & 2 & 1 & Oviparous & Actinopterygia & 0.80 & LC \\
\hline
\end{tabular}




\begin{tabular}{|c|c|c|c|c|c|}
\hline Malacocephalus laevis & 60 & & & & 2 \\
\hline Merlangius merlangus & & 74 & 0.16 & 28 & 2 \\
\hline Myxine glutinosa & 80 & & & 25 & 2 \\
\hline Diplodus cervinus & & 58 & 0.15 & 28.4 & 2 \\
\hline Dentex canariensis & & 85 & 0.15 & 20 & 2 \\
\hline Eutrigla gurnardus & & 46 & 0.16 & 25 & 2 \\
\hline Etmopterus spinax & & 56 & 0.12 & 31 & 11 \\
\hline Thunnus thynnus & & 319 & 0.093 & 104 & 2 \\
\hline Callanthias ruber & 60 & & & & 2 \\
\hline Prionace glauca & & 371 & 0.13 & 221 & 40 \\
\hline Xiphias gladius & & 264 & 0.12 & 145 & 2 \\
\hline Chelon aurata & & 69 & 0.14 & 34 & 2 \\
\hline Pleuronectes platessa & & 48 & 0.23 & 23 & 2 \\
\hline Cyclopterus lumpus & & 53 & 0.26 & 35 & 2 \\
\hline Neoraja iberica & 33 & & & 28 & \\
\hline Beryx decadactylus & & 68 & 0.11 & 21.8 & 2 \\
\hline Lepidopus caudatus & & 195 & 0.23 & & 2 \\
\hline Trachinus draco & & 38 & 0.15 & & 2 \\
\hline Mola mola & & 318 & 0.15 & & 2 \\
\hline Lycodes vahlii & 52 & & & & 2 \\
\hline Pagellus erythrinus & & 47 & 0.084 & 17 & 2 \\
\hline Beryx splendens & & 50 & 0.17 & 33 & 2 \\
\hline Halargyreus johnsonii & 56 & & & & 2 \\
\hline Pegusa lascaris & & 39 & 0.251 & 22 & 2 \\
\hline Macroparalepis affinis & 55 & & & & 2 \\
\hline Lepidion eques & & 42 & 0.15 & 29.5 & 2 \\
\hline Chaunax pictus & 40 & & & & 2 \\
\hline Solea solea & & 39 & 0.32 & 24 & 2 \\
\hline Trigla lyra & & 52 & 0.21 & & 2 \\
\hline
\end{tabular}

$\begin{array}{lllll}2 & \text { Oviparous } & \text { Actinopterygia } & 0.80 & \text { LC } \\ 4 & \text { Oviparous } & \text { Actinopterygia } & 0.78 & \text { LC } \\ 1 & \text { Oviparous } & \text { Agnatha } & 0.80 & \text { LC } \\ 1 & \text { Oviparous } & \text { Actinopterygia } & 0.80 & \text { LC } \\ 4 & \text { Oviparous } & \text { Actinopterygia } & 0.83 & \text { LC } \\ 7 & \text { Oviparous } & \text { Actinopterygia } & 0.83 & \text { LC } \\ 4 & \text { Viviparous } & \text { Elasmobranchii } & 0.83 & \text { NT } \\ 6 & \text { Oviparous } & \text { Actinopterygia } & 0.83 & \text { NT } \\ 4 & \text { Oviparous } & \text { Actinopterygia } & 0.84 & \text { LC } \\ 6 & \text { Viviparous } & \text { Elasmobranchii } & 0.85 & \text { NT } \\ 6 & \text { Oviparous } & \text { Actinopterygia } & 0.85 & \text { LC } \\ 5 & \text { Oviparous } & \text { Actinopterygia } & 0.86 & \text { LC } \\ 3 & \text { Oviparous } & \text { Actinopterygia } & 0.87 & \text { LC } \\ 7 & \text { Oviparous } & \text { Actinopterygia } & 0.92 & \text { NT } \\ 3 & \text { Oviparous } & \text { Elasmobranchii } & 0.97 & \text { LC } \\ 5 & \text { Oviparous } & \text { Actinopterygia } & 0.95 & \text { LC } \\ 5 & \text { Oviparous } & \text { Actinopterygia } & 1.00 & \text { LC } \\ 1 & \text { Oviparous } & \text { Actinopterygia } & 1.07 & \text { LC } \\ 6 & \text { Oviparous } & \text { Actinopterygia } & 1.08 & \text { DD } \\ 2 & \text { Oviparous } & \text { Actinopterygia } & 1.16 & \text { NA } \\ 5 & \text { Oviparous } & \text { Actinopterygia } & 1.14 & \text { LC } \\ 5 & \text { Oviparous } & \text { Actinopterygia } & 1.16 & \text { LC } \\ 5 & \text { Oviparous } & \text { Actinopterygia } & 1.24 & \text { LC } \\ 3 & \text { Oviparous } & \text { Actinopterygia } & 1.22 & \text { LC } \\ 5 & \text { Oviparous } & \text { Actinopterygia } & 1.28 & \text { NA } \\ 5 & \text { Oviparous } & \text { Actinopterygia } & 1.28 & \text { LC } \\ 7 & \text { Oviparous } & \text { Actinopterygia } & 1.29 & \text { LC } \\ 3 & \text { Oviparous } & \text { Actinopterygia } & 1.38 & \text { DD } \\ 2 & \text { Oviparous } & \text { Actinopterygia } & 1.38 & \text { DD }\end{array}$




\begin{tabular}{|c|c|c|c|c|}
\hline Benthodesmus elongatus & 100 & & & \\
\hline Chelon ramada & & 47 & 0.15 & 25 \\
\hline Galeus atlanticus & 46 & & & 36.9 \\
\hline Gaidropsarus vulgaris & & 46 & & 27 \\
\hline Sarpa salpa & & 50 & 0.20 & 29.4 \\
\hline Trachyrincus murrayi & 45 & & & \\
\hline Platichthys flesus & & 39 & 0.25 & 18 \\
\hline Umbrina canariensis & & 57 & 0.26 & \\
\hline Enchelyopus cimbrius & 41 & & & 25 \\
\hline Pagrus pagrus & & 60 & 0.17 & 22.6 \\
\hline Microchirus azevia & & 34 & 0.35 & 23 \\
\hline Chelidonichthys obscurus & 51 & & & \\
\hline Microstomus kitt & & 35 & 0.1955 & 16 \\
\hline Chelidonichthys lucerna & & 52 & 0.25 & 28 \\
\hline Gaidropsarus mediterraneus & 50 & & & \\
\hline Mugil cephalus & & 49 & 0.302 & 33 \\
\hline Lumpenus lampretaeformis & & 48 & 0.205 & 20 \\
\hline Cepola macrophthalma & & 61 & 0.23 & 23.8 \\
\hline Chelidonichthys cuculus & & 41 & 0.24 & 28 \\
\hline Hoplostethus mediterraneus & & 30 & 0.11 & 16.5 \\
\hline Citharus linguatula & & 30 & 0.19 & \\
\hline Chauliodus sloani & 40 & & & \\
\hline Limanda limanda & & 33 & 0.19 & 14 \\
\hline Zeus faber & & 51 & 0.47 & 36 \\
\hline Myoxocephalus scorpioides & 30 & & & \\
\hline Raniceps raninus & 30 & & & \\
\hline Lampanyctus crocodilus & 36 & & & \\
\hline Labrus bergylta & & 44 & 0.103 & \\
\hline Diplodus sargus & & 50 & 0.13 & 21.6 \\
\hline
\end{tabular}

$\begin{array}{lllll}6 & \text { Oviparous } & \text { Actinopterygia } & 1.43 & \text { NA } \\ 5 & \text { Oviparous } & \text { Actinopterygia } & 1.43 & \text { LC } \\ 4 & \text { Oviparous } & \text { Elasmobranchii } & 1.45 & \text { NT } \\ 2 & \text { Oviparous } & \text { Actinopterygia } & 1.50 & \text { LC } \\ 5 & \text { Oviparous } & \text { Actinopterygia } & 1.57 & \text { LC } \\ 2 & \text { Oviparous } & \text { Actinopterygia } & 1.64 & \text { LC } \\ 3 & \text { Oviparous } & \text { Actinopterygia } & 1.66 & \text { LC } \\ 4 & \text { Oviparous } & \text { Actinopterygia } & 1.72 & \text { LC } \\ 2 & \text { Oviparous } & \text { Actinopterygia } & 1.85 & \text { LC } \\ 4 & \text { Oviparous } & \text { Actinopterygia } & 1.86 & \text { LC } \\ 3 & \text { Oviparous } & \text { Actinopterygia } & 1.89 & \text { LC } \\ 4 & \text { Oviparous } & \text { Actinopterygia } & 1.92 & \text { LC } \\ 3 & \text { Oviparous } & \text { Actinopterygia } & 2.00 & \text { LC } \\ 5 & \text { Oviparous } & \text { Actinopterygia } & 2.03 & \text { LC } \\ 4 & \text { Oviparous } & \text { Actinopterygia } & 2.04 & \text { LC } \\ 5 & \text { Oviparous } & \text { Actinopterygia } & 2.08 & \text { LC } \\ 2 & \text { Oviparous } & \text { Actinopterygia } & 2.13 & \text { LC } \\ 4 & \text { Oviparous } & \text { Actinopterygia } & 2.17 & \text { LC } \\ 5 & \text { Oviparous } & \text { Actinopterygia } & 2.19 & \text { LC } \\ 5 & \text { Oviparous } & \text { Actinopterygia } & 2.20 & \text { LC } \\ 3 & \text { Oviparous } & \text { Actinopterygia } & 2.23 & \text { LC } \\ 5 & \text { Oviparous } & \text { Actinopterygia } & 2.51 & \text { LC } \\ 3 & \text { Oviparous } & \text { Actinopterygia } & 2.53 & \text { LC } \\ 4 & \text { Oviparous } & \text { Actinopterygia } & 3.01 & \text { DD } \\ 7 & \text { Oviparous } & \text { Actinopterygia } & 3.11 & \text { NA } \\ 7 & \text { Oviparous } & \text { Actinopterygia } & 3.11 & \text { LC } \\ 5 & \text { Oviparous } & \text { Actinopterygia } & 3.16 & \text { LC } \\ 4 & \text { Oviparous } & \text { Actinopterygia } & 3.16 & \text { LC } \\ 4 & \text { Oviparous } & \text { Actinopterygia } & 3.21 & \text { LC }\end{array}$


Trachyrincus scabrus

Scorpaena porcus

Micromesistius poutassou

Zeugopterus punctatus

Nezumia sclerorhynchus

Argentina silus

Zoarces viviparus

Cyttopsis rosea

Echiodon drummondii

Arnoglossus imperialis

Polymetme corythaeola

Acantholabrus palloni

Sarda sarda

Entelurus aequoreus

Trigloporus lastoviza

Mullus surmuletus

Hippoglossoides platessoides

Chlorophthalmus agassizi

Caranx rhonchus

Polymetme thaeocoryla

Notoscopelus kröyeri

Balistes capriscus

Dicologlossa cuneata

Spondyliosoma cantharus

Lestidiops jayakari

Melanogrammus aeglefinus

Chirolophis ascanii

Nezumia aequalis

Gaidropsarus biscayensis
0.22

0.16

0.24

28

$26 \quad 0.16$

$43 \quad 0.19$

$\begin{array}{ll}35 & 0.37\end{array}$

31

30

25

26

25

60

$81 \quad 0.35$

$28 \quad 0.409$

$51 \quad 0.19$

$25 \quad 0.34$

$19 \quad 0.24$

$45 \quad 0.30$

22

$15 \quad 0.20$

$46 \quad 0.33$

$27 \quad 0.42$

$41 \quad 0.11$

20

18

$50 \quad 0.30$

$16 \quad 0.18$
37

13.8

21

2

$15 \quad 2$

$29 \quad 2$

175

2

2

2

2

2

2

$16 \quad 2$

$17 \quad 2$

$15 \quad 2$

2

2

2

2

2

2

$\begin{array}{ll}15 & 2 \\ 20 & 2\end{array}$

$\begin{array}{ll}20 & 2 \\ & 2\end{array}$

$20 \quad 2$

$4.5 \quad 2$
4 Oviparous

7 Oviparous

5 Oviparous

3 Oviparous

5 Oviparous

4 Oviparous

7 Viviparous

5 Oviparous

2 Oviparous

3 Oviparous

5 Oviparous

5 Oviparous

6 Oviparous

6 Oviparous

7 Oviparous

4 Oviparous

3 Oviparous

5 Oviparous

4 Oviparous

5 Oviparous

5 Oviparous

4 Oviparous

3 Oviparous

4 Oviparous

5 Oviparous

4 Oviparous

5 Oviparous

5 Oviparous

4 Oviparous
Actinopterygia

Actinopterygia

Actinopterygia

Actinopterygia

Actinopterygia

Actinopterygia

Actinopterygia

Actinopterygia

Actinopterygia

Actinopterygia

Actinopterygia

Actinopterygia

Actinopterygia

Actinopterygia

Actinopterygia

Actinopterygia

Actinopterygia

Actinopterygia

Actinopterygia

Actinopterygia

Actinopterygia

Actinopterygia

Actinopterygia

Actinopterygia

Actinopterygia

Actinopterygia

Actinopterygia

Actinopterygia

Actinopterygia
3.24 LC

3.31 LC

3.45 LC

3.49 LC

3.62 LC

$4.05 \mathrm{NA}$

4.19 LC

4.21 DD

4.23 LC

5.78 LC

5.92 LC

$6.33 \mathrm{LC}$

6.84 LC

7.11 LC

7.24 DD

7.63 DD

7.65 LC

7.86 LC

7.92 DD

7.97 LC

8.04 NA

8.10 DD

8.14 LC

8.48 LC

8.97 LC

8.87 LC

10.41 LC

10.53 LC

10.55 LC 
Pholis gunnellus

Trisopterus luscus

Belone belone

Coelorinchus caelorhincus

Gadiculus argenteus

Leptoclinus maculatus

Echiichthys vipera

Sebastes viviparus

Serranus cabrilla

Blennius ocellaris

Trachurus picturatus

Trisopterus esmarkii

Microchirus variegatus

Agonus cataphractus

Anthias anthias

Aphia minuta

Arctozenus risso

Argentina sphyraena

Argyropelecus olfersii

Arnoglossus laterna

Arnoglossus rueppelii

Arnoglossus thori

Atherina presbyter

Auxis rochei rochei

Bathysolea profundicola

Benthosema glaciale

Boops boops

$\begin{array}{rrrr}22 & 0.1986 & 10 & 2 \\ 47 & 0.21 & 18 & 2 \\ 58 & 0.468 & 45 & 2 \\ 39 & 0.15 & & 2 \\ & & & 2 \\ & & & 2 \\ 15 & 0.33 & & 2 \\ 36 & 0.07 & 15 & 2 \\ 39 & 0.10 & 15.4 & 2 \\ & & & 2 \\ 50 & 0.11 & 27.7 & 2 \\ 20 & 0.71 & 14 & 2 \\ 21 & 0.374 & 14 & 2\end{array}$

2 Oviparous

Actinopterygia

Actinopterygia

Actinopterygia

Actinopterygia

Actinopterygia

Actinopterygia

Actinopterygia

Actinopterygia

Actinopterygia

Actinopterygia

Actinopterygia

Actinopterygia

Actinopterygia
11.00 LC

11.28 LC

12.64 LC

12.85 DD

12.96 LC

14.20 LC

15.69 LC

16.88 LC

16.97 LC

17.13 LC

17.59 LC

18.27 LC

18.33 LC

$\begin{array}{rrrrr} & 15 & 0.475 & & 2 \\ 30 & & & & 2 \\ & 9 & 2.23 & 5 & 2 \\ & & & & 2 \\ 9 & & 0.28 & & 2 \\ & 16 & & & 2 \\ & & & & 2 \\ & 18 & 0.84 & 11 & 2 \\ & 16 & 0.138 & 7 & 2 \\ & 46 & 0.70 & & 2 \\ & 15 & 0.58 & & 2 \\ & 7 & 0.36 & & 2 \\ & 36 & 0.17 & 14.3 & 2\end{array}$

$\begin{array}{ll}7 & \text { Oviparous } \\ 4 & \text { Oviparous } \\ 6 & \text { Oviparous } \\ 6 & \text { Oviparous } \\ 4 & \text { Oviparous } \\ 5 & \text { Oviparous } \\ 3 & \text { Oviparous } \\ 3 & \text { Oviparous } \\ 3 & \text { Oviparous } \\ 6 & \text { Oviparous } \\ 6 & \text { Oviparous } \\ 3 & \text { Oviparous } \\ 6 & \text { Oviparous } \\ 4 & \text { Oviparous }\end{array}$

Actinopterygia

20.00 LC

Actinopterygia

20.00 LC

20.00 LC

Actinopterygia

20.00 LC

20.00 LC

Actinopterygia

20.00 LC

20.00 LC

Actinopterygia

20.00 DD

20.00 DD

20.00 LC

20.00 LC

20.00 LC

20.00 LC

Actinopterygia

Actinopterygia

20.00 LC 
Buenia jeffreysii

Buglossidium luteum

Callionymus lyra

Capros aper

Centrolabrus exoletus

Ceratoscopelus maderensis

Clupea harengus

Cottunculus microps

Crystallogobius linearis

Ctenolabrus rupestris

Dentex maroccanus

Diplecogaster bimaculata

Diplodus annularis

Diplodus bellottii

Diplodus vulgaris

Engraulis encrasicolus

Epigonus denticulatus

Gadella maraldi

Gaidropsarus argentatus

Gaidropsarus macropthalmus

Gasterosteus aculeatus

Gobius gasteveni

Gobius paganellus

Hygophum benoiti

Hymenocephalus italicus

Icelus bicornis

Labrus mixtus

Lepidotrigla cavillone

Lepidotrigla dieuzeidei

Leptagonus decagonus
6

$\begin{array}{rrrr} & & & 2 \\ 12 & 0.54 & 8 & 2 \\ 18 & 0.55 & & 2 \\ 17 & 0.145 & 10 & 2 \\ 13 & 0.69 & 11 & 2 \\ & & & 2 \\ 30 & 0.33 & 26 & 2\end{array}$

2

$32 \quad 0.18$

6

\section{5}

.

0.27

0.27

$28 \quad 0.39$

$23 \quad 0.44$

20

30

35

25

8

8

0.67

12

14

0.73

6

19

$6 \quad 0.18$

$2.7 \quad 2$

35

17

20

21
4 Oviparous

1 Oviparous

7 Oviparous

6 Oviparous

5 Oviparous

5 Oviparous

6 Oviparous

4 Oviparous

6 Oviparous

4 Oviparous

4 Oviparous

4 Oviparous

4 Oviparous

4 Oviparous

4 Oviparous

6 Oviparous

4 Oviparous

4 Oviparous

4 Oviparous

4 Oviparous

6 Oviparous

2 Oviparous

2 Oviparous

6 Oviparous

4 Oviparous

4 Oviparous

4 Oviparous

2 Oviparous

4 Oviparous

4 Oviparous
Actinopterygia

Actinopterygia

Actinopterygia

Actinopterygia

Actinopterygia

Actinopterygia

Actinopterygia

Actinopterygia

Actinopterygia

Actinopterygia

Actinopterygia

Actinopterygia

Actinopterygia

Actinopterygia

Actinopterygia

Actinopterygia

Actinopterygia

Actinopterygia

Actinopterygia

Actinopterygia

Actinopterygia

Actinopterygia

Actinopterygia

Actinopterygia

Actinopterygia

Actinopterygia

Actinopterygia

Actinopterygia

Actinopterygia

Actinopterygia
20.00 LC

20.00 LC

20.00 LC

20.00 LC

20.00 LC

20.00 LC

20.00 LC

20.00 DD

20.00 LC

20.00 LC

20.00 LC

20.00 LC

20.00 LC

20.00 LC

20.00 LC

20.00 LC

20.00 LC

20.00 LC

20.00 LC

20.00 LC

20.00 LC

20.00 LC

20.00 LC

20.00 LC

20.00 LC

20.00 LC

20.00 LC

20.00 LC

20.00 LC

20.00 LC 


\begin{tabular}{|c|c|c|c|c|c|}
\hline Lesueurigobius friesii & 13 & & & & 2 \\
\hline Lesueurigobius sanzi & 11 & & & & 2 \\
\hline Lithognathus mormyrus & & 35 & 0.26 & 16 & 2 \\
\hline Lycenchelys sarsi & 20 & & & 11 & 2 \\
\hline Lycodes gracilis & & 15 & 0.39 & & 2 \\
\hline Macroramphosus scolopax & & 15.7 & 0.654 & & 2 \\
\hline Maurolicus muelleri & & 6 & 0.88 & & \\
\hline Micrenophrys lilljeborgii & & 7 & & & 2 \\
\hline Microchirus boscanion & 20 & & & & 2 \\
\hline Monochirus hispidus & & 17 & 0.24 & 9 & 2 \\
\hline Myctophum punctatum & & 9 & 0.32 & 5 & 2 \\
\hline Myoxocephalus scorpius & & 27 & 0.83 & 12 & 2 \\
\hline Naucrates ductor & & 44 & 2.15 & & 2 \\
\hline Nerophis lumbriciformis & 15 & & & & 2 \\
\hline Ophidion barbatum & 25 & & & & 2 \\
\hline Pagellus acarne & & 34 & 0.21 & 16 & 2 \\
\hline Parablennius gattorugine & 30 & & & & 2 \\
\hline Phrynorhombus norvegicus & & 12 & & & 2 \\
\hline Pomatoschistus lozanoi & 8 & & & & 2 \\
\hline Pomatoschistus norvegicus & 8 & & & & 2 \\
\hline Pterycombus brama & 46 & & & & 2 \\
\hline Pungitius pungitius & & 5.7 & 0.13 & & 2 \\
\hline Sardina pilchardus & & 19 & 0.4 & 15 & 2 \\
\hline Scomber colias & & 56 & 0.19 & 21.6 & 2 \\
\hline Scomber scombrus & & 43 & 0.26 & & 2 \\
\hline Scomberesox saurus & & 35 & 0.71 & & 2 \\
\hline Scorpaena loppei & & 11 & 0.53 & & 2 \\
\hline Scorpaena notata & & 18 & 0.43 & & 2 \\
\hline Serranus hepatus & & 15 & 0.36 & 7.8 & 2 \\
\hline Serranus scriba & & 28 & 0.16 & 17.3 & \\
\hline
\end{tabular}

$\begin{array}{lllll}2 & \text { Oviparous } & \text { Actinopterygia } & 20.00 & \text { LC } \\ 2 & \text { Oviparous } & \text { Actinopterygia } & 20.00 & \text { LC } \\ 4 & \text { Oviparous } & \text { Actinopterygia } & 20.00 & \text { LC } \\ 7 & \text { Oviparous } & \text { Actinopterygia } & 20.00 & \text { LC } \\ 4 & \text { Oviparous } & \text { Actinopterygia } & 20.00 & \text { LC } \\ 5 & \text { Oviparous } & \text { Actinopterygia } & 20.00 & \text { LC } \\ 6 & \text { Oviparous } & \text { Actinopterygia } & 20.00 & \text { LC } \\ 4 & \text { Oviparous } & \text { Actinopterygia } & 20.00 & \text { LC } \\ 3 & \text { Oviparous } & \text { Actinopterygia } & 20.00 & \text { LC } \\ 3 & \text { Oviparous } & \text { Actinopterygia } & 20.00 & \text { LC } \\ 5 & \text { Oviparous } & \text { Actinopterygia } & 20.00 & \text { LC } \\ 7 & \text { Oviparous } & \text { Actinopterygia } & 20.00 & \text { LC } \\ 5 & \text { Oviparous } & \text { Actinopterygia } & 20.00 & \text { LC } \\ 4 & \text { Oviparous } & \text { Actinopterygia } & 20.00 & \text { LC } \\ 4 & \text { Oviparous } & \text { Actinopterygia } & 20.00 & \text { LC } \\ 4 & \text { Oviparous } & \text { Actinopterygia } & 20.00 & \text { LC } \\ 4 & \text { Oviparous } & \text { Actinopterygia } & 20.00 & \text { LC } \\ 3 & \text { Oviparous } & \text { Actinopterygia } & 20.00 & \text { LC } \\ 2 & \text { Oviparous } & \text { Actinopterygia } & 20.00 & \text { LC } \\ 2 & \text { Oviparous } & \text { Actinopterygia } & 20.00 & \text { LC } \\ 6 & \text { Oviparous } & \text { Actinopterygia } & 20.00 & \text { LC } \\ 5 & \text { Oviparous } & \text { Actinopterygia } & 20.00 & \text { LC } \\ 6 & \text { Oviparous } & \text { Actinopterygia } & 20.00 & \text { NT } \\ 6 & \text { Oviparous } & \text { Actinopterygia } & 20.00 & \text { LC } \\ 6 & \text { Oviparous } & \text { Actinopterygia } & 20.00 & \text { LC } \\ 6 & \text { Oviparous } & \text { Actinopterygia } & 20.00 & \text { LC } \\ 7 & \text { Oviparous } & \text { Actinopterygia } & 20.00 & \text { LC } \\ 7 & \text { Oviparous } & \text { Actinopterygia } & 20.00 & \text { LC } \\ 4 & \text { Oviparous } & \text { Actinopterygia } & 20.00 & \text { LC } \\ & \text { Actinopterygia } & 20.00 & \text { LC }\end{array}$


Sprattus sprattus

Stomias boa

Symphodus bailloni

Symphodus melops

Symphodus roissali

Taurulus bubalis

Taurulus lilljeborgi

Trachurus mediterraneus

Trachurus trachurus

Triglops murrayi

Trisopterus minutus

Xenodermichthys copei

Zeugopterus regius
14

0.63

$\begin{array}{rr} & 2 \\ & 2 \\ 16 & 2 \\ 5 & 2 \\ & 2 \\ & 2 \\ & 2 \\ 20 & 2 \\ 22 & 2 \\ & 2 \\ 13 & 2 \\ & 2 \\ & 2 \\ & 2\end{array}$

32

20

$21 \quad 0.31$

$13 \quad 0.59$

$19 \quad 0.251$

$43 \quad 0.21$

$40 \quad 0.21$

$18 \quad 0.22$

$24 \quad 0.40$

20
6 Oviparous

6 Oviparous

4 Oviparous

4 Oviparous

4 Oviparous

7 Oviparous

7 Oviparous

6 Oviparous

6 Oviparous

7 Oviparous

4 Oviparous

6 Oviparous

3 Oviparous
Actinopterygia

Actinopterygia

Actinopterygia

Actinopterygia

Actinopterygia

Actinopterygia

Actinopterygia

Actinopterygia

Actinopterygia

Actinopterygia

Actinopterygia

Actinopterygia

Actinopterygia
20.00 LC

20.00 LC

20.00 LC

20.00 LC

20.00 LC

20.00 LC

20.00 NA

20.00 LC

20.00 LC

20.00 LC

20.00 LC

20.00 LC

20.00 LC 
Table S3. References for life history parameters used.

Species

Acantholabrus palloni

Agonus cataphractus

Alopias vulpinus

Amblyraja hyperborea

Amblyraja radiata
Source of $\boldsymbol{L}_{\max }$ or $\boldsymbol{L}_{\infty} \& K$

Source of $\boldsymbol{L}_{\text {mat }}$

Source of $L_{\min }$

Gomon, M.F. and P. Forsyth, 1990. Labridae. p. 868-882. In J.C. Quero, J.C. Hureau, C. Karrer,

A. Post and L. Saldanha (eds.) Check-list of the fishes of the eastern tropical Atlantic

(CLOFETA). JNICT, Lisbon, SEI, Paris; and UNESCO, Paris. Vol. 2.

Pauly, D., 1978. A preliminary compilation of fish length growth parameters. Ber. Inst. Meereskd. Christian-Albrechts-Univ. Kiel (55):1-200.

Natanson, L.J., Hamady, L.L. and Gervelis, B.J., 2016. Analysis of bomb radiocarbon data for common thresher sharks, Alopias vulpinus, in the northwestern Atlantic Ocean with revised growth curves. Environmental biology of fishes, 99(1), pp.39-47.

Last, P.R., W.T. White, M.R. de Carvalho, B. Séret, M.F.W. Stehmann and G.J.P. Naylor, 2016. Rays of the world. CSIRO Publishing, Comstock Publishing Associates. i-ix + 1-790.

Jennings, S., S.P.R. Greenstreet and J.D. Reynolds, 1999. Structural change in an exploited fish community: a consequence of differential fishing effects on species with
Smith, S.E., R.C. Rasmussen, D.A. Ramon and G.M. Cailliet, 2008. The biology and ecology of thresher sharks (Alopiidae). p. 60-68. In M.D. Camhi, E.K. Pikitch and E.A. Babcock (eds) Sharks of the open ocean. Blackwell Publishing, Oxford, UK, 502 p.

Jennings, S., S.P.R. Greenstreet and J.D. Reynolds, 1999. Structural change in an exploited fish community: a consequence of differential fishing effects on species with contrasting life histories. J. Animal Ecol. 68:617-627.
Moreno, J.A., Parajúa, J.I. and Morón, J. 1989. Biolgía reproductiva y fenología de Alopias vulpinus (Bonnaterre, 1788)

(Squaliformes: Alopiidae) en el Atlántico nororiental y Mediterráneo occidental.

Scientia Marina 53(1): 37-46.

Last, P.R., W.T. White, M.R. de Carvalho, B. Séret, M.F.W. Stehmann and G.J.P.

Naylor, 2016. Rays of the world. CSIRO

Publishing, Comstock Publishing Associates. i-ix + 1-790.

Last, P.R., W.T. White, M.R. de Carvalho, B. Séret, M.F.W. Stehmann and G.J.P. Naylor, 2016. Rays of the world. CSIRO Publishing, Comstock Publishing Associates. i-ix + 1-790. 
contrasting life histories. J. Animal Ecol. 68:617-627.

Anarhichas lupus

Anarhichas minor

Anthias anthias

Aphia minuta

Arctozenus risso

Argentina silus
Liao, Y.-Y. and M.C. Lucas, 2000. Growth, diet and metabolism of common wolf-fish in the North Sea, a fast-growing population. J. Fish Biol. 56(4):810-825.

Gunnarsson, A., Hjörleifsson, E., Thórarinsson, K. and Marteinsdóttir, G., 2008. Growth, maturity and fecundity of female spotted wolffish Anarhichas minor in Icelandic waters. Journal of Fish Biology, 73(6), pp.1393-1406.

Bauchot, M.-L., 1987. Poissons osseux. p. $891-$

1421. In W. Fischer, M.L. Bauchot and M.

Schneider (eds.) Fiches FAO d'identification

pour les besoins de la pêche. (rev. 1).

Méditerranée et mer Noire. Zone de pêche 37.

Vol. II. Commission des Communautés

Européennes and FAO, Rome.

Iglesias, M., E.B. Brothers and B. Morales-Nin, 1997. Validation of daily increment deposition in otoliths. Age and growth determination of Aphia minuta (Pisces: Gobiidae) from the northwest Mediterranean. Mar. Biol. 129(2):279-287.

Muus, B.J. and J.G. Nielsen, 1999. Sea fish. Scandinavian Fishing Year Book, Hedehusene, Denmark. 340 p.

Bergstad, O.A., 1993. Distribution, population structure, growth, and reproduction of the
Gunnarsson, A., Hjörleifsson, E.,

Thorarinsson, $\mathrm{K}$. and Marteinsdottir, $\mathrm{G}$ 2006. Growth, maturity and fecundity of wolffish Anarhichas lupus L. in Icelandic waters. Journal of fish biology, 68(4),

pp.1158-1176

Gunnarsson, A., Hjörleifsson, E.,

Thórarinsson, K. and Marteinsdóttir, G., 2008. Growth, maturity and fecundity of female spotted wolffish Anarhichas minor in Icelandic waters. Journal of Fish Biology, 73(6), pp.1393-1406.

Iglesias, M. and B. Morales-Nin, 2001. Life cycle of the pelagic goby Aphia minuta

(Pisces: Gobiidae). Sci. Mar. 65(3):183-192.

Johannesen, A \& Monstad, T. 2003.Distribution, growth and exploitation 
greater silver smelt, Argentina silus (Pisces, Argentinidae), of the Skagerrak and the northeastern North Sea. ICES J. mar. Sci. 50:129-143.

Argentina sphyraena

Argyropelecus olfersi

Argyrosomus regius

Arnoglossus imperialis

Arnoglossus laterna

Arnoglossus rueppeli
Lee, J.Y., 1963. Les Argentines du Golfe du Lion, Argentina sphyraena L., Argentina

leioglossa Val. Rev. Trav. Inst. Pêches Marit. 27(1-4):189-202.

Reiner, F., 1996. Catálogo dos peixes do arquipélago de Cabo Verde. Publ. Avuls. Inst. Port. Invest. Mar. 2:339 p.

González-Quirós, R., J. del Árbol, M.M. GarcíaPacheco, A.J. Silva-García, J.M. Naranjo and B. Morales-Nin, 2011. Life-history of the meagre Argyrosomus regius in the Gulf of Cádiz (SW Iberian Peninsula). Fish. Res. 109(1):140-149.

Schneider, W., 1990. FAO species identification sheets for fishery purposes. Field guide to the commercial marine resources of the Gulf of Guinea. Prepared and published with the support of the FAO Regional Office for Africa. Rome: FAO. 268 p.

Deniel, C., 1990. Comparative study of growth of flatfishes on the west coast of Brittany. Journal of Fish Biology, 37(1), pp.149-166.

Nielsen, J.G., 1986. Bothidae. p. 1294-1298. In P.J.P. Whitehead, M.-L. Bauchot, J.-C. Hureau, J. Nielsen and E. Tortonese (eds.) Fishes of the North-eastern Atlantic and the Mediterranean. UNESCO, Paris. Vol. 3. of greater silver smelt (Argentina silus (Ascanius, 1775)) in Norwegian waters 1980-83. Journal of Northwest Atlantic Fishery Science 31:319-332

González-Quirós, R., J. del Árbol, M.M. García-Pacheco, A.J. Silva-García, J.M. Naranjo and B. Morales-Nin, 2011. Lifehistory of the meagre Argyrosomus regius in the Gulf of Cádiz (SW Iberian Peninsula). Fish. Res. 109(1):140-149.

Deniel, C., 1990. Comparative study of growth of flatfishes on the west coast of Brittany. Journal of Fish Biology, 37(1), pp.149-166. 
Arnoglossus thori

Atherina presbyter

Balistes capriscus

Bathysolea profundicola

Belone belone

Benthodesmus elongatus
Deniel, C., 1990. Comparative study of growth of flatfishes on the west coast of Brittany. J.

Fish Biol. 37(1):149-166

Pombo, L., Elliott, M. and Rebelo, J.E., 2005.

Ecology, age and growth of Atherina boyeri and Atherina presbyter in the Ria de Aveiro, Portugal. Cybium, 29(1), pp.47-55.

Valeiras, X., D. Macias, M.J. Gomez, L. Lema, S. Garcia-Barcelona, J.M. De Urbinqa Ortiz and

J.M. de la Serna, 2008. Age and growth of bullet tuna (Auxis rochei) in the western Mediterranean Sea. Collect. Vol. Sci. Pap. ICCAT 62(5):1629-1637

Burton, M.L., J.C. Potts, D.R. Carr, M. Cooper and J. Lewis, 2015. Age, growth, and mortality of gray triggerfish (Balistes capriscus) from the southeastern United States. Fish. Bull.

113(1):27-39.

Cau, A. and A. Deiana, 1983. Reproduction et accroissement dans quelques Soleidae de la Mediterranee du centre occidental. Rapp.

Comm. int. Mer Médit. 28(5):61-62.

Potoschi, A., A. Cefali and G. Cavallaro, 1983. Notes on the biology of Belone belone gracilis Lowe 1839, captured in the area of the strait of Messina. Mem. Biol. Mar. Ocean. 13:19-36.

Nakamura, I., 1995. Trichiuridae. Peces sables, cintillas. p. 1638-1642. In W. Fischer, F. Krupp,
Félix, P.M., Vinagre, C. and Cabral, H.N., 2011. Life-history traits of flatfish in the Northeast Atlantic and Mediterranean Sea. Journal of Applied Ichthyology, 27(1) pp.100-111.

Moreno, T., Castro, J.J. and Socorro, J., 2005. Reproductive biology of the sand smelt Atherina presbyter Cuvier, 1829 (Pisces: Atherinidae) in the central-east Atlantic. Fisheries research, 72(1), pp.121131.

Muus, B.J. and J.G. Nielsen, 1999. Sea fish. Scandinavian Fishing Year Book, Hedehusene, Denmark. 340 p. 
W. Schneider, C. Sommer, K.E. Carpenter and V. Niem (eds.) Guia FAO para Identification de Especies para lo Fines de la Pesca. Pacifico Centro-Oriental. 3 Vols. FAO, Rome.

Benthosema glaciale

Beryx decadactylus

Beryx splendens

Blennius ocellaris

Boops boops
Gjøsaeter, J., 1973. Age, growth and mortality of the myctophid fish Benthosema glaciale (Reinhardt) from western Norway. Sarsia 52:1 14.

Krug, H., D. Carvalho\& J.A. González2010. Age and growth of the alfonsino Beryx decadactylus(Cuvier, 1829) from the Azores, Madeira and Canary Islands, based on historical data. Arquipelago. Life and Marine Sciences 28:25-31

Rico, V., J.M. Lorenzo, J.A. González, H.M. Krug, A. Mendonça, E. Gouveia and M.A. Dias, 2001. Age and growth of the alfonsino Beryx splendens Lowe, 1834 from the Macronesian archipelagos. Fish. Res. 49(3):233-240.

Bauchot, M.-L., 1987. Poissons osseux. p. 8911421. In W. Fischer, M.L. Bauchot and M.

Schneider (eds.) Fiches FAO d'identification pour les besoins de la pêche. (rev. 1).

Méditerranée et mer Noire. Zone de pêche 37

Vol. II. Commission des Communautés

Européennes and FAO, Rome.

Zuniga, L.R., 1967. Estudio del crecimiento de Boops boops (L.) del Levante Español. Inv.

Pesq. 31(3):383-418
Estácio, S. and Krug, H., 2001. Aspects of the reproduction of six exploited demersal fish species in the Azores Archipelago. Fauna and Flora of the Atlantic Islands. Arquipelago, Life and Marine Sciences, suppl 2(Part B), p.83.

Lehodey, P. and R. Grandperrin, 1996. Age and growth of the alfonsino Beryx splendens over the seamounts off New Caledonia. Mar. Biol. 125:249-258.

Layachi, M., M.H, Idrissi, M. Ramdani, F. Sahnouni and R. Flower, 2015. Growth and reproduction of the Bogue Boops boops $L$. 1758 in the editerranean coastal area 


\section{Brama brama}

Brosme brosme

Buenia jeffreysii

Callanthias ruber

Callionymus lyra
Lobo, C. and K. Erzini, 2001. Age and growth of Ray's bream (Brama brama) from the south of Portugal. Fish. Res. 51(2-3):343-347.

ICES, 2018. Report of the Working Group on Biology and Assessment of Deep-sea Fisheries Resources. Stock annex: Tusk (Brosme brosme) in subareas 4 and 7-9, and in divisions 3.a, 5.b, 6.a, and 12.b (Northeast Atlantic)

Miller, P.J., 1986. Gobiidae. p. 1019-1085. In P.J.P. Whitehead, M.-L. Bauchot, J.-C. Hureau, J. Nielsen and E. Tortonese (eds.) Fishes of the North-eastern Atlantic and the Mediterranean. Volume 3. UNESCO, Paris.

Deniel, C., 1990. Comparative study of growth of flatfishes on the west coast of Brittany. Journal of Fish Biology, 37(1), pp.149-166.

Edwards, A., 1990. Fish and fisheries of Saint Helena Island. Centre for Tropical Coastal Management Studies, University of Newcastle upon Tyne, England.

Beverton, R.J.H. and S.J. Holt, 1959. A review of the lifespans and mortality rates of fish in nature, and their relation to growth and other physiological characteristics. p. 142-180. In G.E.W. Wolstenholme and M. O'Connor (eds.) CIBA Foundation colloquia on ageing: the lifespan of animals. volume 5. J \& A Churchill Ltd, London. between Nador and Saidia (Morocco). Bull. Inst. Sci., Rabat, Sec. Sci. Vie, (37).

ICES, 2018. Report of the Working Group on Deep Sea Fisheries. Stock annex: Tusk (Brosme brosme) in subareas 4 and 7-9, and in divisions 3.a, 5.b, 6.a, and 12.b (Northeast Atlantic)

Muus, B.J. and J.G. Nielsen, 1999. Sea fish. Scandinavian Fishing Year Book, Hedehusene, Denmark. 340 p. 
Capros aper

Caranx rhonchus

Cepola macrophthalma

Ceratoscopelus maderensis

Hulley, P.A., 1990. Myctophidae. p. 398-467. In

Cetorhinus maximus J.C. Quero, J.C. Hureau, C. Karrer, A. Post and L. Saldanha (eds.) Check-list of the fishes of the eastern tropical Atlantic (CLOFETA). JNICT, Lisbon; SEI; Paris; and UNESCO, Paris. Vol. 1.

Hüssy, K., Coad, J.O., Farrell, E.D., Clausen, L.W. and Clarke, M.W., 2012. Sexual dimorphism in size, age, maturation, and growth characteristics of boarfish (Capros aper) in the Northeast Atlantic. ICES Journal of Marine Science, 69(10), pp.1729-1735.

CECAF, 1979. Report of the ad hoc working group on west African coastal pelagic fish from Mauritania to Liberia $\left(26^{\circ} \mathrm{N}\right.$ to $\left.5^{\circ} \mathrm{N}\right)$.

CECAF/ECAF Ser. 78/10. FAO, UNDP, Rome.

$161 \mathrm{p}$.

Sayer, M. D. J., R. N. Gibson, and R. J. A. Atkinson. "Growth, diet and condition of corkwing wrasse and rock cook on the west coast of Scotland." Journal of Fish Biology 49, no. 1 (1996): 76-94.

Stergiou, K.I., P. Economidis and A. Sinis, 1992. Age, growth and mortality of red bandfish, Cepola macrophthalma (L.), in the western Aegean Sea (Greece). J. Fish Biol. 40(3):395418.

Pauly, D., 2002. Growth and mortality of the basking shark Cetorhinus maximus and their implications for management of whale sharks Rhincodon typus. IUCN SSC SHARK SPECIALIST
Hüssy, K., Coad, J.O., Farrell, E.D., Clausen, L.W. and Clarke, M.W., 2012. Sexual dimorphism in size, age, maturation, and growth characteristics of boarfish (Capros aper) in the Northeast Atlantic. ICES Journa of Marine Science, 69(10), pp.1729-1735.

Sayer, M. D. J., R. N. Gibson, and R. J. A. Atkinson. "Growth, diet and condition of corkwing wrasse and rock cook on the west coast of Scotland." Journal of Fish Biology 49, no. 1 (1996): 76-94.

Stergiou, K.I., E.D. Christou, D.

Georgopoulous, A. Zenetos and C. Souvermezoglou, 1997. The Hellenic seas: physics, chemistry, biology and fisheries. $p$. 415-538. In A.D. Ansell, R.N. Gibson and M. Barnes (eds.). Oceanography and marine biology: an annual review. UCL Press.

Pauly, D., 2002. Growth and mortality of the basking shark Cetorhinus maximus and their implications for management of whale sharks Rhincodon typus. IUCN SSC SHARK
Sund, O. 1943. Et brugdelbarsel. Naturen 67: 285-286. 
Chauliodus sloani

\section{Chaunax pictus}

Chelidonichthys cuculus

Chelidonichthys lucerna

Chelidonichthys obscurus
GROUP. Elasmobranch biodiversity,

conservation and management: Proceedings of the International Seminar and Workshop,

Sabah, Malaysia, 1997. IUCN: Gland

Switzerland and Cambridge, UK:199-208.

Masuda, H., K. Amaoka, C. Araga, T. Uyeno and T. Yoshino, 1984. The fishes of the Japanese Archipelago. Vol. 1. Tokai University Press, Tokyo, Japan. 437 p. (text).

Smith, M.M., 1986. Chaunacidae. p. 369-370.

In M.M. Smith and P.C. Heemstra (eds.)

Smiths' sea fishes. Springer-Verlag, Berlin.

Marriott, A.L., J.W. Latchford and I.D.

McCarthy, 2010. Population biology of the red gurnard (Aspitrigla cuculus L.; Triglidae) in the

inshore waters of Eastern Anglesey and Northwest Wales. J. Appl. Ichthyol. 26:504-

512.

McCarthy, I.D. and Marriott, A.L., 2018. Age, growth and maturity of tub gurnard (Chelidonichthys lucerna Linnaeus 1758 ; Triglidae) in the inshore coastal waters of Northwest Wales, UK. Journal of applied ichthyology, 34(3), pp.581-589.

Wilhelms, I., 2013. Atlas of length-weigh relationships of 93 fish and crustacean species from the North Sea and the North-East Atlantic (No. 12). Johann Heinrich von Thünen

Institute, Federal Research Institute for Rura Areas, Forestry and Fisheries, 552 p.
SPECIALIST GROUP. Elasmobranch

biodiversity, conservation and

management: Proceedings of the International Seminar and Workshop

Sabah, Malaysia, 1997. IUCN: Gland,

Switzerland and Cambridge, UK:199-208.

Marriott, A.L., J.W. Latchford and I.D.

McCarthy, 2010. Population biology of the red gurnard (Aspitrigla cuculus L.; Triglidae) in the inshore waters of Eastern Anglesey and Northwest Wales. J. Appl. Ichthyol. 26:504-512.

McCarthy, I.D. and Marriott, A.L., 2018. Age, growth and maturity of tub gurnard (Chelidonichthys lucerna Linnaeus 1758; Triglidae) in the inshore coastal waters of Northwest Wales, UK. Journal of applied ichthyology, 34(3), pp.581-589. 
Chelon aurata

Chelon labrosus

Chimaera monstrosa

Chirolophis ascanii

Chlorophthalmus agassizi
Campillo, A., 1992. Les pêcheries françaises de Méditeranée: synthèse des connaissances.

Institut Francais de Recherche pour

l'Exploitation de la Mer, France. 206 p.

Richter, H., 1995. Age and growth of thick-

lipped grey mullet Chelon labrosus (Risso 1826)(Pisces: Mugilidae) in strangford Lough,

Co Down. The Irish Naturalists' Journal, pp.134-139.

Autem, M., 1979. Contribution à l'étude biologique des zones de dilution du littoral méditerranéen: estuaires, étangs lagunaires. USTL Montpellier (France). 343 p. Thèse 3ième cycle.

Moura, T., I. Figueiredo, P.B. Machado and L.S. Gordo, 2004. Growth pattern and reproductive strategy of the holocephalan Chimaera montrosa along the Portuguese continental slope. J. Mar. Biol. Ass. U.K. 84(4):801-804.

Makushok, V.M., 1986. Stichaeidae. p. 1122 1123. In P.J.P. Whitehead, M.-L. Bauchot, J.-C. Hureau, J. Nielsen and E. Tortonese (eds.) Fishes of the North-eastern Atlantic and the Mediterranean. UNESCO, Paris. Vol. 3.

Maiorano, P., L. Sion, R. Carlucci, F. Capezzuto, A. Giove, G. Costantino, M. Panza, G. D'Onghia and $A$. Tursi, 2010. The demersal faunal

assemblage of the north-western Ionian Sea

(central Mediterranean): current knowledge
Campillo, A., 1992. Les pêcheries françaises de Méditeranée: synthèse des

connaissances. Institut Francais de

Recherche pour l'Exploitation de la Mer,

France. 206 p.

Campillo, A., 1992. Les pêcheries

françaises de Méditeranée: synthèse des connaissances. Institut Francais de

Recherche pour l'Exploitation de la Mer, France. $206 p$

Moura, T., I. Figueiredo, P.B. Machado and L.S. Gordo, 2004. Growth pattern and reproductive strategy of the holocephalan Chimaera monstrosa along the Portuguese continental slope. J. Mar. Biol. Ass. U.K. 84(4):801-804
Stehmann, M. and D.L. Bürkel, 1984.

Chimaeridae. p. 212-215. In P.J.P.

Whitehead, M.-L. Bauchot, J.-C. Hureau, J. Nielsen and E. Tortonese (eds.) Fishes of the north-eastern Atlantic and the Mediterranean. UNESCO, Paris. vol. 1. 
and perspectives. Chemistry and Ecology 26 (Supplement):219-240.

Citharus linguatula

Coelorinchus caelorhincus

Conger conger

Coryphaenoides rupestris
Moutopoulos, D.K., A. Ramfos, A. Mouka and G. Katselis, 2013. Length-weight relations of 34 fish species caught by small-scale fishery in Korinthiakos Gulf (Central Greece). Acta Ichthyol. Piscat. 43(1):57-64.

Jennings, S. and Beverton, R.J.H., 1991. Intraspecific variation in the life history tactics of Atlantic herring (Clupea harengus L.) stocks. ICES Journal of Marine Science, 48(1), pp.117125.

Massuti, E., B. Morales-Nin and C. Stefanescu, 1995. Distribution and biology of five grenadier fish (Pisces: Macrouridae) from the upper and middle slope of the northwestern Mediterranean. Deep-Sea Research 42(3):307330.

Correia, A.T., Manso, S. and Coimbra, J., 2009. Age, growth and reproductive biology of the European conger eel (Conger conger) from the Atlantic Iberian waters. Fisheries Research, 99(3), pp.196-202.

Bergstad, O.A., 1990. Distribution, population structure, growth and reproduction of the roundnose grenadier Coryphaenoides rupestris (Pisces: Macrouridae) in the deep waters of the Skagerrak. Mar. Biol. 107:25-39.
Jennings, S. and Beverton, R.J.H., 1991. Intraspecific variation in the life history tactics of Atlantic herring (Clupea harengus L.) stocks. ICES Journal of Marine Science, 48(1), pp.117-125.

Correia, A., Antunes, C. and Coimbra, J., 2002. Aspects of the early life history of the European conger eel (Congerconger) inferred from the otolith microstructure of metamorphic larvae. Marine Biology, 140(1), pp.165-173.
Bergstad, O.A., 1990. Distribution, population structure, growth and reproduction of the roundnose grenadier Coryphaenoides rupestris (Pisces: Macrouridae) in the deep waters of the Skagerrak. Mar. Biol. 107:25-39. 
Cottunculus microps

Crystallogobius linearis

Ctenolabrus rupestris

Cyclopterus lumpus

Cyttopsis rosea

Dalatias licha

Dasyatis pastinaca
Muus, B.J. and J.G. Nielsen, 1999. Sea fish.

Scandinavian Fishing Year Book, Hedehusene,

Denmark. 340 p.

Miller, P.J., 1986. Gobiidae. p. 1019-1085. In

P.J.P. Whitehead, M.-L. Bauchot, J.-C. Hureau,

J. Nielsen and E. Tortonese (eds.) Fishes of the

North-eastern Atlantic and the Mediterranean.

Volume 3. UNESCO, Paris.

Pauly, D., 1978. A preliminary compilation of fish length growth parameters. Ber. Inst. Meereskd. Christian-Albrechts-Univ. Kiel (55):1-200.

Kasper, J.M., Bertelsen, B., Ólafsson, H.G., Holst, J.C., Sturlaugsson, J. and Jónsson, S.P., 2014. Observations of growth and postspawning survival of lumpfish Cyclopterus lumpus from mark-recapture studies. Journal of Fish Biology, 84(6), pp.1958-1963.

Karrer, C. and A. Post, 1990. Zeidae. p. 631 633. In J.C. Quero, J.C. Hureau, C. Karrer, A. Post and L. Saldanha (eds.) Check-list of the fishes of the eastern tropical Atlantic (CLOFETA). JNICT, Lisbon; SEI, Paris; and UNESCO, Paris. Vol. 2.

da Silva, H.N., 1988. Growth and reproduction of Kitefin shark Dalatias lici (Bonn, 1788) in Azorean waters. ICES CM.1988/G:21

Yigin, C.C. and Ismen, A., 2012. Age, growth and reproduction of the common stingray,
Albert, O.T., Torstensen, E., Bertelsen, B., Jonsson, S.T., Pettersen, I.H. and Holst, J.C. 2002. Age-reading of lumpsucker

(Cyclopteruslumpus) otoliths: dissection, interpretation and comparison with length frequencies. Fisheries Research, 55(1-3), pp.239-252.

da Silva, H.N., 1988. Growth and reproduction of Kitefin shark Dalatias lici (Bonn, 1788) in Azorean waters. ICES CM.1988/G:22

Yigin, C.C. and Ismen, A., 2012. Age, growth and reproduction of the common stingray, da Silva, H.N., 1988. Growth and reproduction of Kitefin shark Dalatias lici (Bonn, 1788) in Azorean waters. ICES CM.1988/G:23

Ismen, A. 2003. Age, growth, reproduction and food of common 
Dasyatis pastinaca from the North Aegean Sea. Marine Biology Research, 8(7), pp.644-653.

Dasyatis tortonesei

Deania calcea

Deania profundorum

Dentex canariensis

Dentex gibbosus
McEachran, J.D. and C. Capapé, 1984.

Dasyatidae. p. 197-202. In P.J.P. Whitehead, M.-L. Bauchot, J.-C. Hureau, J. Nielsen and E. Tortonese (eds.) Fishes of the north-eastern Atlantic and Mediterranean. UNESCO, Paris. Vol. 1.

Clarke, M.W., Connolly, P.L. and Bracken, J.J., 2002. Catch, discarding, age estimation, growth and maturity of the squalid shark Deania calceus west and north of Ireland. Fisheries Research, 56(2), pp.139-153.

Bass, A.J., L.J.V. Compagno and P.C. Heemstra, 1986. Squalidae. p. 49-62. In M.M. Smith and P.C. Heemstra (eds.) Smiths' sea fishes. Springer-Verlag, Berlin.

Mennes, F., 1985. Multispecies assessment of fish stocks off the Western Sahara region with emphasis on the family Sparidae. Fishbyte 3(3):5-10.

Pajuelo, J.G. and J.M. Lorenzo, 1995. Biologica parameters reflecting the current state of the exploited pink dentex Dentex gibbosus (Pisces: Sparidae) populations off the Canary Islands. S Afr. J. Mar. Sci. 16:311-319.
Dasyatis pastinaca from the North Aegean Sea. Marine Biology Research, 8(7), pp.644 653.

Yigin, C.C. and Ismen, A. 2012. Age, growth and reproduction of the common stingray, Daysatis pastinaca from the North Aegean Sea. Marine Biology Research 8: 644-653.

Clarke, M.W., Connolly, P.L. and Bracken, J.J., 2002. Catch, discarding, age estimation, growth and maturity of the squalid shark Deania calceus west and north of Ireland. Fisheries Research, 56(2), pp.139-153.

Bauchot, M.-L. and J.-C. Hureau, 1986. Sparidae. p. 883-907. In P.J.P. Whitehead, M.-L. Bauchot, J.-C. Hureau, J. Nielsen and E. Tortonese (eds.) Fishes of the northeastern Atlantic and the Mediterranean. volume 2. UNESCO, Paris.

Pajuelo, J.G. and J.M. Lorenzo, 1995.

Biological parameters reflecting the current state of the exploited pink dentex Dentex gibbosus (Pisces: Sparidae) populations off the Canary Islands. S. Afr. J. Mar. Sci. 16:311-319. stingray (Dasyatis pastinacaL., 1758) in Iskenderun Bay, the eastern

Mediterranean. Fisheries Research 60:169-176

Ismen, A. 2003. Age, growth, reproduction and food of common stingray (Dasyatis pastinaca L., 1758) in Iskenderun Bay, the eastern Mediterranean. Fisheries Research 60: 169-176.

Cox, G. and M. Francis, 1997. Sharks and rays of New Zealand. Canterbury Univ. Press, Univ. of Canterbury. 68 p. 
Dentex maroccanus

Dicentrarchus labrax

Dicentrarchus punctatus

Dicologlossa cuneata

Diplecogaster bimaculata

Diplodus annularis

Diplodus bellottii
Lamrini, A. and A. Bouymajjane, 2002. Biologie de Dentex maroccanus (Valenciennes, 1830) dans la région de Safi. Actes Institute Agronomique et Veterinaire (Maroc) 22(1):1118.

ICES, 2016. Report of the Working Group for the Celtic Seas Ecoregion (WGCSE), 4-13 May 2016, Copenhagen, Denmark. ICES CM 2016/ACOM:13. 1343 pp.

Gravier, R., 1961. Les Bars (Loups) du Maroc Atlantique Morone labrax (Linné) et Morone punctata (Bloch). Rev. Trav. Inst. Pêches Marit. 25(3):281-292

Félix, P.M., C. Vinagre and H.N Cabral, 2011. Life-history traits of flatfish in the Northeast Atlantic and Mediterranean Sea. J. Appl. Ichthyol. 27:100-111.

Muus, B.J. and J.G. Nielsen, 1999. Sea fish. Scandinavian Fishing Year Book, Hedehusene, Denmark. 340 p.

Pajuelo, J.G. and J.M. Lorenzo, 2001. Biology of the annular seabream, Diplodus annularis (Sparidae), in coastal waters of the Canary Islands. J. Appl. Ichthyol. 17:121-125.

Mennes, F., 1985. Multispecies assessment of fish stocks off the Western Sahara region with emphasis on the family Sparidae. Fishbyte $3(3): 5-10$.
Lamrini, A. and A. Bouymajjane, 2002.

Biologie de Dentex maroccanus

(Valenciennes, 1830) dans la région de Safi. Actes Institute Agronomique et Veterinaire (Maroc) 22(1):11-18.

Pawson, M.G., S. Kupschus and G.D. Pickett 2007. The status of sea bass (Dicentrarchus labrax) stocks around England and Wales, derived using a separable catch-at-age model, and implications for fisheries

management. ICES J. Mar. Sci. 64:346-356.

Félix, P.M., C. Vinagre and H.N Cabral,

2011. Life-history traits of flatfish in the Northeast Atlantic and Mediterranean Sea.

J. Appl. Ichthyol. 27:100-111.

Pajuelo, J.G. and J.M. Lorenzo, 2001.

Biology of the annular seabream, Diplodus annularis (Sparidae), in coastal waters of the Canary Islands. J. Appl. Ichthyol. 
Diplodus cervinus

Diplodus sargus

Diplodus vulgaris

Dipturus batis-complex

Dipturus nidarosiensis

Dipturus oxyrinchus
Pajuelo, J.G., J.M. Lorenzo and R. DomínguezSeoane, 2003. Age estimation and growth of the zebra seabream Diplodus cevinus cervinus (Lowe, 1838) on the Canary Islands shelf (Central-east Atlantic). Fish. Res. 62(1):97-103.

Pajuelo, J.G. and Lorenzo, J.M., 2004. Basic characteristics of the population dynamic and state of exploitation of Moroccan white seabream Diplodus sargus cadenati (Sparidae) in the Canarian archipelago. Journal of Applied Ichthyology, 20(1), pp.15-21.

Gonçalves, J.M.S., L. Bentes, R. Coelho, C. Correia, P.G. Lino, C.C. Monteiro, J. Ribeiro and K. Erzini, 2003. Age and growth, maturity, mortality and yield-per-recruit for two banded bream ( Diplodus vulgaris Geoffr.) from the south coast of Portugal. Fish. Res. 62(3):349359

Du Buit, M.H., 1977. Age et croissance de Raja batis et de Raja naevus en Mer Celtique. J. Cons. CIEM 37:261-265.

Muus, B.J. and J.G. Nielsen, 1999. Sea fish Scandinavian Fishing Year Book, Hedehusene, Denmark. 340 p.

Yigin, C. and A. Ismen, 2010. Age, growth, reproduction and feed of longnosed skate,
Pajuelo, J.G., J.M. Lorenzo and R.

Domínguez-Seoane, 2003. Age estimation

and growth of the zebra seabream Diplodus cevinus cervinus (Lowe, 1838) on the

Canary Islands shelf (Central-east Atlantic). Fish. Res. 62(1):97-103.

Pajuelo, J.G. and Lorenzo, J.M., 2004. Basic characteristics of the population dynamic and state of exploitation of Moroccan white seabream Diplodus sargus cadenat (Sparidae) in the Canarian archipelago. Journal of Applied Ichthyology, 20(1), pp.15-21.

Gonçalves, J.M.S., L. Bentes, R. Coelho, C. Correia, P.G. Lino, C.C. Monteiro, J. Ribeiro and K. Erzini, 2003. Age and growth,

maturity, mortality and yield-per-recruit for two banded bream ( Diplodus vulgaris Geoffr.) from the south coast of Portugal Fish. Res. 62(3):349-359

Du Buit, M.H., 1977. Age et croissance de Raja batis et de Raja naevus en Mer Celtique. J. Cons. CIEM 37:261-265.

Follesa, M.C., Cannas, R., Cabiddu, S., Cau, A., Mulas, A., Porcu, C. and Cau, A., 2012. Preliminary observations of the

reproductive biology and diet for the Norwegian skate Dipturus nidarosiensis

(Rajidae) from the central western Mediterranean Sea. Cybium, 36(3), pp.473477.

Yigin, C. and A. Ismen, 2010. Age, growth, reproduction and feed of longnosed skate,
Clark, R.S. 1922. Skates and rays (Raiae) No.1. Egg capsules and young. Journal of the Marine Biological Association of the United Kingdom 12: 577-643.

Last, P.R., W.T. White, M.R. de Carvalho, B. Séret, M.F.W. Stehmann and G.J.P. 
Dipturus oxyrinchus (Linnaeus, 1758) in Saros bay, the north Aegean Sea. J. Appl. Ichthyol. 26(6):913-919.

Echiichthys vipera

Creutzberg, F. and Witte, J.I., 1989. An attempt to estimate the predatory pressure exerted by the lesser weever, Trachinus vipera Cuvier, in the southern North Sea. Journal of Fish Biology, 34(3), pp.429-449.

Echiodon drummondi

Trott, L.B. and J.E. Olney, 1986. Carapidae. p. 1172-1176. In P.J.P. Whitehead, M.-L. Bauchot J.-C. Hureau, J. Nielsen and E. Tortonese (eds.) Fishes of the North-eastern Atlantic and the Mediterranean. UNESCO, Paris. Vol. 3.

Enchelyopus cimbrius

Engraulis encrasicolus

Entelurus aequoreus

Synop. 125(10). Rome: FAO. 442 p.

Silva, A., P. Carrera, J. Massé, A. D. Uriarte, M. B. Santos, P. B. Oliveira, E. Soares, C. Porteiro and Y. Stratoudakis, 2008. Geographic variability of sardine growth across the northeastern Atlantic and the Mediterranean Sea. Fish. Res. 90:56-69.

Muus, B.J. and J.G. Nielsen, 1999. Sea fish
Dipturus oxyrinchus (Linnaeus, 1758) in Saros bay, the north Aegean Sea. J. Appl. Ichthyol. 26(6):913-919.

Jennings, S., Greenstreet, S.P. and Reynolds, J.D., 1999. Structural change in an exploited fish community: a

consequence of differential fishing effects on species with contrasting life histories. Journal of Animal Ecology, 68(3), pp.617-

gadiform fishes known to date. FAO Fish. 627. Scandinavian Fishing Year Book, Hedehusene, Denmark. 340 p.

ICES, 2010. Report of the Workshop on Age reading of European anchovy (WKARA), 9-13 November 2009, Sicily, Italy. ICES CM 2009/ACOM:43. 122 pp.
Naylor, 2016. Rays of the world. CSIRO Publishing, Comstock Publishing Associates. $\mathrm{i}-\mathrm{ix}+1-790$ 
Ephippion guttifer

Cousseau, M.B., 1993. Las especies del orden gadiformes del Atlántico sudamericano comprendido entre $34^{\circ}$ y $55^{\circ} \mathrm{S}$ y relación con las de otras áreas. Frente Marit. 13:7-108.

Epigonus denticulatus

Gon, O., 1986. Apogonidae. p. 546-561. In

M.M. Smith and P.C. Heemstra (eds.) Smiths' sea fishes. Springer-Verlag, Berlin.

Epigonus telescopus

Paulin, C., A. Stewart, C. Roberts and P. McMillan, 1989. New Zealand fish: a complete guide. National Museum of New Zealand Miscellaneous Series No. 19. $279 p$

Etmopterus princeps

Etmopterus pusillus

Etmopterus spinax
Compagno, L.J.V., 1984. FAO Species Catalogue. Vol. 4. Sharks of the world. An annotated and illustrated catalogue of shark species known to date. Part 1 -

Hexanchiformes to Lamniformes. FAO Fish. Synop. 125(4/1):1-249. Rome, FAO.

Coelho, R. and Erzini, K., 2007. Population parameters of the smooth lantern shark, Etmopterus pusillus, in southern Portugal (NE Atlantic). Fisheries Research, 86(1), pp.42-57.

Coelho, R. and Erzini, K., 2008. Life history of a wide-ranging deepwater lantern shark in the north-east Atlantic, Etmopterus spinax (Chondrichthyes: Etmopteridae), with implications for conservation. Journal of Fish Biology, 73(6), pp.1419-1443.
Dunn, M.R., 2009. Review and stock assessment of black cardinal fish (Epigonus telescopus) on the east coast of North Island, New Zealand. New Zealand Fish. Assess. Rep. 2009/39. 55 p.

Cotton, C.F., Grubbs, R.D., Dyb, J.E., Fossen, I. and Musick, J.A., 2015. Reproduction and embryonic development in two species of squaliform sharks, Centrophorus granulosus and Etmopterus princeps: Evidence of matrotrophy?. Deep Sea Research Part II: Topical Studies in Oceanography, 115, pp.41-54.

Coelho, R. and Erzini, K., 2007. Population parameters of the smooth lantern shark, Etmopterus pusillus, in southern Portugal (NE Atlantic). Fisheries Research, 86(1), pp.42-57.

Coelho, R. and Erzini, K., 2008. Life history of a wide-ranging deepwater lantern shark in the north-east Atlantic, Etmopterus spinax (Chondrichthyes: Etmopteridae), with implications for conservation. Journal of Fish Biology, 73(6), pp.1419-1443.
Cotton, C.F., Grubbs, R.D., Dyb, J.E., Fossen, I. and Musick, J.A., 2015. Reproduction and embryonic development in two species of squaliform sharks, Centrophorus granulosus and Etmopterus princeps: Evidence of matrotrophy?. Deep Sea Research Part II: Topical Studies in Oceanography, 115, pp.41-54.

Coelho, R. and Erzini, K., 2008. Life history of a wide-ranging deepwater lantern shark in the north-east Atlantic Etmopterus spinax (Chondrichthyes: Etmopteridae), with implications for 
Eutrigla gurnardus

Gadella maraldi

Gadiculus argenteus

Gadus morhua

Gaidropsarus argentatus

Gaidropsarus biscayensis
McCarthy, I.D., Cant, J. and Marriott, A.L., 2018. Population biology of grey gurnard (Eutrigla gurnardus (L.); Triglidae) in the coastal waters of Northwest Wales. Journal of applied ichthyology, 34(4), pp.896-905.

Cohen, D.M., T. Inada, T. Iwamoto and N. Scialabba, 1990. FAO species catalogue. Vol. 10. Gadiform fishes of the world (Order Gadiformes). An annotated and illustrated catalogue of cods, hakes, grenadiers and other gadiform fishes known to date. FAO Fish. Synop. 125(10). Rome: FAO. 442 p.

Wilhelms, I., 2013. Atlas of length-weight relationships of 93 fish and crustacean species from the North Sea and the North-East Atlantic (No. 12). Johann Heinrich von Thünen Institute, Federal Research Institute for Rural Areas, Forestry and Fisheries, 552 p.

Daan, N., 1974. Growth of the North Sea cod Gadus morhua. Neth. J. Sea Res. 8(1):27-48.

Muus, B.J. and J.G. Nielsen, 1999. Sea fish.

Scandinavian Fishing Year Book, Hedehusene, Denmark. 340 p.

Cohen, D.M., T. Inada, T. Iwamoto and N. Scialabba, 1990. FAO species catalogue. Vol. 10. Gadiform fishes of the world (Order Gadiformes). An annotated and illustrated
McCarthy, I.D., Cant, J. and Marriott, A.L., 2018. Population biology of grey gurnard (Eutrigla gurnardus (L.); Triglidae) in the coastal waters of Northwest Wales. Journal of applied ichthyology, 34(4), pp.896-905. conservation. Journal of Fish Biology, 73(6), pp.1419-1443.
Oosthuizen, E. and Daan, N., 1974. Egg

fecundity and maturity of North Sea cod, Gadus morhua. Netherlands Journal of Sea Research, 8(4), pp.378-397. 
catalogue of cods, hakes, grenadiers and other gadiform fishes known to date. FAO Fish.

Synop. 125(10). Rome: FAO. 442 p.

Gaidropsarus

macropthalmus

Galeorhinus galeus

Galeus atlanticus
Cohen, D.M., T. Inada, T. Iwamoto and N. Scialabba, 1990. FAO species catalogue. Vol. 10. Gadiform fishes of the world (Order Gadiformes). An annotated and illustrated catalogue of cods, hakes, grenadiers and other gadiform fishes known to date. FAO Fish. Synop. 125(10). Rome: FAO. 442 p.

Cohen, D.M., T. Inada, T. Iwamoto and N. Scialabba, 1990. FAO species catalogue. Vol. 10. Gadiform fishes of the world (Order Gadiformes). An annotated and illustrated catalogue of cods, hakes, grenadiers and other gadiform fishes known to date. FAO Fish. Synop. 125(10). Rome: FAO. 442 p.

Cohen, D.M., T. Inada, T. Iwamoto and N. Scialabba, 1990. FAO species catalogue. Vol. 10. Gadiform fishes of the world (Order Gadiformes). An annotated and illustrated catalogue of cods, hakes, grenadiers and other gadiform fishes known to date. FAO Fish. Synop. 125(10). Rome: FAO. 442 p.

Ferreira, B.P. and C.M. Vooren, 1991. Age, growth, and structure of vertebra in the school shark Galeorhinus galeus (Linnaeus, 1758) from southern Brazil. Fish. Bull. 89:19-31.

Rey, J., Coelho, R., Lloris, D., Séret, B. and Gi de Sola, L., 2010. Distribution pattern of

Galeus atlanticus in the Alboran Sea (south
Morato, T., Afonso, P., Santos, R.S., Krug, H.M. and Nash, R.D.M., 2003. The reproduction, age and growth of the spotted rockling. Journal of fish biology, 62(6), pp.1450-1455
Rey, J., Coelho, R., Lloris, D., Séret, B. and Gil de Sola, L., 2010. Distribution pattern of Galeus atlanticus in the Alboran Sea (south 
western Mediterranean) and some sexual character comparison with Galeus

melastomus. Marine Biology Research, 6(4), pp.364-372.

Galeus melastomus

Costa, M.E., Erzini, K. and Borges, T.C. 2005. Reproductive biology of the blackmouth catshark, Galeus melastomus (Chondrichthyes: Scyliorhinidae) off the south coast of Portugal. Journal of the Marine Biological Association of the United Kingdom 85(5): 1173-1183.

Gasterosteus aculeatus

Yershov, P. and A. Sukhotin, 2015. Age and growth of marine three-spined stickleback in the White Sea 50 years after a population collapse. Polar Biology 38(11):1813-1823.

Glyptocephalus cynoglossus

Isen, R., Gutvik, O.K., Nilssen, E.M. and Hopkins, C.C.E., 1991. Population parameters of the witch flounder, Glyptocephalus cynoglossus (L.)(Pisces: Pleuronectidae), from Malangen, northern Norway. Fisheries research, 12(3), pp.259-278.

Gnathophis mystax

Gobius gasteven

Bauchot, M.-L., 1987. Poissons osseux. p. 8911421. In W. Fischer, M.L. Bauchot and M. Schneider (eds.) Fiches FAO d'identification pour les besoins de la pêche. (rev. 1).

Méditerranée et mer Noire. Zone de pêche 37.

Vol. II. Commission des Communautés

Européennes and FAO, Rome.

Miller, P.J., 1990. Gobiidae. p. 925-951. In J.C. Quero, J.C. Hureau, C. Karrer, A. Post and L. Saldanha (eds.) Check-list of the fishes of the

western Mediterranean) and some sexual character comparison with Galeus

melastomus. Marine Biology Research, 6(4), pp.364-372.

Moore, D.M., F.C. Neat and I.D. McCArthy, 2013. Population biology and ageing of the deep water sharks Galeus melastomus, Centroselachus crepidater and Apristurus aphyodes from the Rockall Trough, northeast Atlantic. J. Mar. Biol. Assoc. U.K. 93(7):1941-1950.

Nilsen, R., Gutvik, O.K., Nilssen, E.M. and Hopkins, C.C.E., 1991. Population parameters of the witch flounder,

Glyptocephalus cynoglossus (L.)(Pisces: Pleuronectidae), from Malangen, northern Norway. Fisheries research, 12(3), pp.259278. 
Gobius paganellus

Helicolenus dactylopterus

Hexanchus griseus

Hippoglossoides platessoides NS eastern tropical Atlantic (CLOFETA). JNICT,

Lisbon, SEI, Paris; and UNESCO, Paris. Vol. 2.

Azevedo, J.M.N. and A.M.V. Simas, 2000. Age and growth, reproduction and diet of sublittoral population of the rock goby Gobius paganellus (Teleostei: Gobiidae). Hydrobiologia 440:129-135.

Cohen, D.M., T. Inada, T. Iwamoto and N. Scialabba, 1990. FAO species catalogue. Vol. 10. Gadiform fishes of the world (Order Gadiformes). An annotated and illustrated catalogue of cods, hakes, grenadiers and other gadiform fishes known to date. FAO Fish.

Synop. 125(10). Rome: FAO. 442 p.

Kelly, C.J., Connolly, P.L. and Bracken, J.J., 1999. Age estimation, growth, maturity, and distribution of the bluemouth rockfish Helicolenus d. dactylopterus (Delaroche 1809) from the Rockall Trough. ICES Journal of Marine Science, 56(1), pp.61-74.

Compagno, L.J.V., 1984. FAO Species Catalogue. Vol. 4. Sharks of the world. An annotated and illustrated catalogue of shark species known to date. Part 1 -

Hexanchiformes to Lamniformes. FAO Fish. Synop. 125(4/1):1-249. Rome, FAO.

Jennings, S., Greenstreet, S.P. and Reynolds, J.D., 1999. Structural change in an exploited fish community: a consequence of differential fishing effects on species with contrasting life
Azevedo, J.M.N. and A.M.V. Simas, 2000. Age and growth, reproduction and diet of sublittoral population of the rock goby Gobius paganellus (Teleostei: Gobiidae). Hydrobiologia 440:129-135.

Kelly, C.J., Connolly, P.L. and Bracken, J.J. 1999. Age estimation, growth, maturity, and distribution of the bluemouth rockfish Helicolenus d. dactylopterus (Delaroche 1809) from the Rockall Trough. ICES Journal of Marine Science, 56(1), pp.61-74.

Ebert, D.A., 2002. Some observations on the reproductive biology of the sixgill shark Hexanchus griseus (Bonnaterre, 1788) from South African waters. African Journal of Marine Science, 24, pp.359-363.

Jennings, S., Greenstreet, S.P. and Reynolds, J.D., 1999. Structural change in an exploited fish community: a

consequence of differential fishing effects on species with contrasting life histories.
Cox, G. and M. Francis, 1997. Sharks and rays of New Zealand. Canterbury Univ. Press, Univ. of Canterbury. 68 p. 
histories. Journal of Animal Ecology, 68(3), pp.617-627.

Hippoglossus hippoglossus

Haug, T., 1990. Biology of the Atlantic halibut, Hippoglossus hippoglossus (L. 1758). Adv. Mar. Biol. 26:26-35.

Hoplostethus mediterraneus D'Onghia, G., A. Tursi, C.A. Marano and M Basanisi, 1998. Life history traits of

Hoplostethus mediterraneus (Pisces:

Beryciformes) from the North-Western Ionian Sea (Mediterranean Sea). J. Mar. Biol. Ass. U.K. 78:321-339.

Hygophum benoiti

Hymenocephalus italicus

Hulley, P.A., 1990. Myctophidae. p. 398-467. In J.C. Quero, J.C. Hureau, C. Karrer, A. Post and L. Saldanha (eds.) Check-list of the fishes of the eastern tropical Atlantic (CLOFETA). JNICT,

Lisbon; SEI; Paris; and UNESCO, Paris. Vol. 1.

Maiorano, P., L. Sion, R. Carlucci, F. Capezzuto, A. Giove, G. Costantino, M. Panza, G. D'Onghia and $A$. Tursi, 2010. The demersal faunal assemblage of the north-western Ionian Sea (central Mediterranean): current knowledge and perspectives. Chemistry and Ecology 26 (Supplement):219-240.

Icelus bicornis

Fedorov, V.V., 1986. Cottidae. p. 1243-1260. In P.J.P. Whitehead, M.-L. Bauchot, J.-C. Hureau, J. Nielsen and E. Tortonese (eds.) Fishes of the North-eastern Atlantic and the Mediterranean. UNESCO, Paris. Vol. 3.

Labrus bergylta

Dipper, F.A., C.R. Bridges and A. Menz, 1977.
Journal of Animal Ecology, 68(3), pp.617627.

Haug, T., 1990. Biology of the Atlantic halibut, Hippoglossus hippoglossus (L. 1758). Adv. Mar. Biol. 26:26-35.

D'Onghia, G., A. Tursi, C.A. Marano and M. Basanisi, 1998. Life history traits of Hoplostethus mediterraneus (Pisces: Beryciformes) from the North-Western Ionian Sea (Mediterranean Sea). J. Mar. Biol. Ass. U.K. 78:321-339.

Massuti, E., B. Morales-Nin and C. Stefanescu, 1995. Distribution and biology of five grenadier fish (Pisces: Macrouridae) from the upper and middle slope of the northwestern Mediterranean. Deep-Sea Research 42(3):307-330

Age, growth and feeding in the ballan wrasse, 
Labrus bergylta Ascanius 1767. J. Fish Biol. 11:105-120.

Labrus mixtus

Bauchot, M.-L., 1987. Poissons osseux. p. 8911421. In W. Fischer, M.L. Bauchot and M. Schneider (eds.) Fiches FAO d'identification pour les besoins de la pêche. (rev. 1).

Méditerranée et mer Noire. Zone de pêche 37.

Vol. Il. Commission des Communautés

Européennes and FAO, Rome.

Lamna nasus

Natanson, L.J., Mello, J.J. and Campana, S.E., 2002. Validated age and growth of the porbeagle shark (Lamna nasus) in the western North Atlantic Ocean. Fishery Bulletin, 100(2), pp.266-278.

Lampanyctus crocodilus

Lepidion eques

Lepidopus caudatus J.C. Quero, J.C. Hureau, C. Karrer, A. Post and L. Saldanha (eds.) Check-list of the fishes of the eastern tropical Atlantic (CLOFETA). JNICT, Lisbon; SEI; Paris; and UNESCO, Paris. Vol. 1.

Magnússon, J.V., 1998. Age, maturity and other biological parameters of two morid species Lepidion eques (Günther, 1887) and Antimora rostrata Günther, 1878, in Icelandic waters. ICES C.M. 1998/0:32. 22 p.

Tuset, V., González, J.A., Santana, J.I., Lopez,
Quignard, J.-P. and A. Pras, 1986. Labridae. p. 919-942. In P.J.P. Whitehead, M.-L.

Bauchot, J.-C. Hureau, J. Nielsen and E.

Tortonese (eds.) Fishes of the north-eastern

Atlantic and the Mediterranean. UNESCO

Paris. Vol. 2.

Jensen, C.F., Natanson, L.J., Pratt Jr, H.L., Kohler, N. and Campana, S.E., 2002. The reproductive biology of the porbeage shark (Lamna nasus) in the western North Atlantic Ocean. Fishery Bulletin, 100(4) pp.727-738.

Hulley, P.A., 1990. Myctophidae. p. 398-467. In A.M. and Diaz, M.G., 2006. Reproductive pattern and growth in Lepidopus caudatus (Osteichthyes, Trichiuridae) from the Canary
Magnússon, J.V., 1998. Age, maturity and other biological parameters of two morid species Lepidion eques (Günther, 1887) and Antimora rostrata Günther, 1878, in Icelandic waters. ICES C.M. 1998/O:32. 22 p.
Jensen, C.F., Natanson, L.J., Pratt Jr, H.L., Kohler, N. and Campana, S.E., 2002. The reproductive biology of the porbeage shark (Lamna nasus) in the western North Atlantic Ocean. Fishery Bulletin, 100(4), pp.727-738. 
islands (Eastern-Central Atlantic). Electronic Journal of Ichthyology, 1, pp.26-37.

Lepidorhombus bosci

Landa, J., Perez, N. and Piñeiro, C., 2002.

Growth patterns of the four spot megrim

(Lepidorhombus boscii) in the northeast

Atlantic. Fisheries Research, 55(1-3), pp.141-

152.

Lepidorhombus whiffiagonis

Macdonald, P., Angus, C.H. and Marshall, C.T., 2013. Spatial variation in life history

characteristics of common megrim

(Lepidorhombus whiffiagonis) on the Northern

Shelf. Journal of sea research, 75, pp.62-68.

Lepidotrigla cavillone

Campillo, A., 1992. Les pêcheries françaises de

Méditeranée: synthèse des connaissances.

Institut Francais de Recherche pour

I'Exploitation de la Mer, France. 206 p.

Lepidotrigla dieuzeidei

Bauchot, M.-L., 1987. Poissons osseux. p. 891-

1421. In W. Fischer, M.L. Bauchot and M.

Schneider (eds.) Fiches FAO d'identification

pour les besoins de la pêche. (rev. 1).

Méditerranée et mer Noire. Zone de pêche 37.

Vol. II. Commission des Communautés

Européennes and FAO, Rome.

Leptagonus decagonus
Andriashev, A.P., 1986. Agonidae. p. 1265

1268. In P.J.P. Whitehead, M.-L. Bauchot, J.-C.

Hureau, J. Nielsen and E. Tortonese (eds.)

Fishes of the North-eastern Atlantic and the

Mediterranean. volume 3. UNESCO, Paris.
Macdonald, P., Angus, C.H. and Marshall, C.T., 2013. Spatial variation in life history characteristics of common megrim (Lepidorhombus whiffiagonis) on the Northern Shelf. Journal of sea research, 75 pp.62-68.

Colloca, F., M. Cardinale and G.D.

Ardizzone, 1997. Biology, spatial

distribution and population dynamics of

Lepidotrigla cavillone (Pisces: Triglidae) in the central Tyrrhenian Sea. Fish. Res.

32(1):21-32 
Leptoclinus maculatus

Lestidiops jayakari

Lesueurigobius friesi

Lesueurigobius sanzi

Leucoraja circularis

Leucoraja fullonica

Leucoraja naevus

Limanda limanda
Muus, B.J. and J.G. Nielsen, 1999. Sea fish.

Scandinavian Fishing Year Book, Hedehusene,

Denmark. 340 p.

Post, A., 1986. Paralepididae. p. 274-278. In M.M. Smith and P.C. Heemstra (eds.) Smiths' sea fishes. Springer-Verlag, Berlin.

Muus, B.J. and J.G. Nielsen, 1999. Sea fish.

Scandinavian Fishing Year Book, Hedehusene, Denmark. 340 p.

Miller, P.J., 1990. Gobiidae. p. 925-951. In J.C. Quero, J.C. Hureau, C. Karrer, A. Post and L.

Saldanha (eds.) Check-list of the fishes of the eastern tropical Atlantic (CLOFETA). JNICT,

Lisbon, SEI, Paris; and UNESCO, Paris. Vol. 2.

Stehmann, M., 1990. Rajidae. p. 29-50. In J.C. Quero, J.C. Hureau, C. Karrer, A. Post and L. Saldanha (eds.) Check-list of the fishes of the eastern tropical Atlantic. Junta Nacional de Investigaçao Cientifica e Tecnológica, Lisbon, Portugal. Vol. 1

Muus, B.J. and J.G. Nielsen, 1999. Sea fish.

Scandinavian Fishing Year Book, Hedehusene, Denmark. 340 p.

Gallagher, M.J., C.P. Nolan and F. Jeal, 2005. Age, growth and maturity of the commercia ray species from the Irish Sea. J. Northw. Atl. Fish. Sci., 35: 47-66.

Rijnsdorp, A.D., A.D. Vethaak and P.I. Van Leeuwan, 1992. Population biology of dab
Maia, C., K. Erzini, B. Serra-Pereira and I. Figueiredo, 2012. Reproductive biology of cuckoo ray Leucoraja naevus. J. Fish Biol. 81:1285-1296.

Rijnsdorp, A.D., A.D. Vethaak and P.I. Van Leeuwan, 1992. Population biology of dab
Clark, R.S., 1922. Rays and Skates (Raiœ) No. 1.-Egg-Capsules and Young. Journal of the Marine Biological Association of the United Kingdom, 12(4), pp.578-643 
Limanda limanda in the southeastern North Sea. Mar. Ecol. Prog. Ser. 91:19-35.

Monteiro, P., Bentes, L., Coelho, R., Correia, C., Erzini, K., Lino, P.G., Ribeiro, J. and Gonçalves, J.M., 2010. Age and growth, mortality and reproduction of the striped sea bream, Lithognathus mormyrus Linnaeus 1758, from the south coast of Portugal (Algarve). Marine Biology Research, 6(1), pp.53-65.

Lophius budegassa

Lophius piscatorius

Lumpenus lampretaeformis fish length growth parameters. Ber. Inst. Meereskd. Christian-Albrechts-Univ. Kiel (55):1-200

Lycenchelys sarsi

Muus, B.J. and J.G. Nielsen, 1999. Sea fish. Scandinavian Fishing Year Book, Hedehusene, Denmark. 340 p.

Lycodes gracilis

Nash, R.D., 1986. Aspects of the general
Limanda limanda in the southeastern North Sea. Mar. Ecol. Prog. Ser. 91:19-35.

Monteiro, P., Bentes, L., Coelho, R., Correia,

C., Erzini, K., Lino, P.G., Ribeiro, J. and Gonçalves, J.M., 2010. Age and growth, mortality and reproduction of the striped sea bream, Lithognathus mormyrus Linnaeus 1758, from the south coast of Portugal (Algarve). Marine Biology Research, 6(1), pp.53-65.

Duarte, R., M. Azevedo, J. Landa and P. Pereda, 2001. Reproduction of the anglerfish (Lophius budegassa Spinola and Lophius piscatorius Linnaeus) from the Atlantic Iberian coast. Fish. Res. 51(2-

3):349-361.

Farina, A.C., Azevedo, M., Landa, J., Duarte R., Sampedro, P., Costas, G., Torres, M.A. and Cañás, L., 2008. Lophius in the world: a synthesis on the common features and life strategies. ICES Journal of Marine Science, 65(7), pp.1272-1280

Muus, B.J. and J.G. Nielsen, 1999. Sea fish. Scandinavian Fishing Year Book, Hedehusene, Denmark. 340 p.

Nash, R.D. and Geffen, A.J., 2005. Aspects of the general biology of Sars' eelpout, Lycenchelys sarsi (Collett, 1871)(Pisces, Zoarcidae), in Oslofjorden, Norway. Marine Biology Research, 1(1), pp.33-38. biology of Vahl's eelpout, Lycodes vahlii gracilis 
M. Sars, 1867 (Pisces, Zoarcidae), in

Oslofjorden, Norway. Sarsia, 71(3-4), pp.289-

296.

Lycodes vahlii

Macroparalepis affinis

Andriashev, A.P., 1986. Zoarcidae. p. 11301150. In P.J.P. Whitehead, M.-L. Bauchot, J.-C. Hureau, J. Nielsen and E. Tortonese (eds.) Fishes of the North-eastern Atlantic and the Mediterranean. volume 3. UNESCO, Paris.

Post, A., 1986. Paralepididae. p. 274-278. In M.M. Smith and P.C. Heemstra (eds.) Smiths' sea fishes. Springer-Verlag, Berlin.

Macroramphosus scolopax

Borges, L., 2000. Age and growth of the snipefish, Macrorhamphosus spp., in the Portuguese continental waters. Journal of the Marine Biological Association of the United Kingdom, 80(1), pp.147-153.

Macrourus berglax

Murua, H., 2003. Population structure, growth and reproduction of roughhead grenadier on the Flemish Cap and Flemish Pass. Journal of Fish Biology, 63(2), pp.356-373

Malacocephalus laevis

Iwamoto, T., 1986. Macrouridae. p. 330-341. In M.M. Smith and P.C. Heemstra (eds.) Smiths' sea fishes. Springer-Verlag, Berlin.

Maurolicus mueller

Gjøsaeter, J. and K. Kawaguchi, 1980. A review of the world resources of mesopelagic fish.

FAO Fish. Tech. Pap. No. 193. FIRM/TI93. 151

Melanogrammus aeglefinus

Baudron, A.R., Needle, C.L. and Marshall, C.T., 2011. Implications of a warming North Sea for
Murua, H., 2003. Population structure, growth and reproduction of roughhead grenadier on the Flemish Cap and Flemish Pass. Journal of Fish Biology, 63(2), pp.356374

Baudron, A.R., Needle, C.L. and Marshall, C.T., 2011. Implications of a warming North Sea for the growth of haddock 
the growth of haddock Melanogrammus aeglefinus. Journal of Fish biology, 78(7) pp.1874-1889.

Merlangius merlangus

Ross, S.D., Nielsen, J.R., Gislason, H., Nielsen, A. and Andersen, N.G., 2018. Growth and food consumption of whiting Merlangius merlangus. Journal of fish biology, 93(2), pp.334-343.

Merluccius merluccius

Micrenophrys lilljeborgii

Microchirus azevia

Microchirus boscanion
ICES, 2017. Report of the Working Group for the Bay of Biscay and the Iberic waters Ecoregion(WGBIE). Stock annex: Hake (Merluccius merluccius) in divisions 8.c and 9.a, Southern stock.

Fedorov, V.V., 1986. Cottidae. p. 1243-1260. In P.J.P. Whitehead, M.-L. Bauchot, J.-C. Hureau, J. Nielsen and E. Tortonese (eds.) Fishes of the North-eastern Atlantic and the Mediterranean. UNESCO, Paris. Vol. 3.

Félix, P.M., Vinagre, C. and Cabral, H.N., 2011. Life-history traits of flatfish in the Northeast Atlantic and Mediterranean Sea. Journal of Applied Ichthyology, 27(1), pp.100-111.

Schneider, W., 1990. FAO species identification sheets for fishery purposes. Field guide to the commercial marine resources of the Gulf of Guinea. Prepared and published with the support of the FAO Regional Office for Africa. Rome: FAO. 268 p.
Melanogrammus aeglefinus. Journal of Fish biology, 78(7), pp.1874-1889.

Jennings, S., J.D. Reynolds and S.C. Mills, 1998. Life history correlates of responses to fisheries exploitation. Proc. R. Soc. London B 265:333-339

Murua, H., 2010. The biology and fisheries of European hake, Merluccius merluccius, in the north-east Atlantic. In Advances in marine biology (Vol. 58, pp. 97-154).

Academic Press.

Félix, P.M., Vinagre, C. and Cabral, H.N. 2011. Life-history traits of flatfish in the Northeast Atlantic and Mediterranean Sea. Journal of Applied Ichthyology, 27(1), pp.100-111. 
Microchirus variegatus

Micromesistius poutassou

Microstomus kitt

Mola mola

Molva dypterygia

Molva macrophthalma
Deniel, C., 1990. Comparative study of growth of flatfishes on the west coast of Brittany. J.

Fish Biol. 37(1):149-166

Estimated from ICES. 2017. Report of the Working Group on Widely Distributed Stocks (WGWIDE),

30 August -5 September 2017, ICES

Headquarters, Copenhagen, Denmark. ICES CM 2017/ACOM:23. 1111 pp.

King, P.A., Hannan, J.F., McGrath, D. and Veldon, M., 2006. Population dynamics, age, growth and maturity of lemon sole Microstomus kitt (Walbaum 1792) sampled between 2000-2002 off the west coast of Ireland. Marine Institute.

Nakatsubo, T. and H. Hirose, 2007. Growth of captive ocean funfish, Mola mola. The Agriculture, Forestry and Fisheries Research Information and Technology Center. Http://www.affrc.go.jp

Pauly, D., 1978. A preliminary compilation of fish length growth parameters. Ber. Inst. Meereskd. Christian-Albrechts-Univ. Kiel (55):1-200.

Postel, E. and M.-H. Du Buit, 1964. Liste des poissons observés à la criée de Concarneau en fin Juillet-début Août 1964. Tailles maximales
Deniel, C., 1990. Comparative study of growth of flatfishes on the west coast of Brittany. J. Fish Biol. 37(1):149-167

Estimated from ICES. 2017. Report of the Working Group on Widely Distributed Stocks (WGWIDE)

30 August -5 September 2017, ICES

Headquarters, Copenhagen, Denmark. ICES CM

2017/ACOM:23. 1111 pp.

Cohen, D.M., T. Inada, T. Iwamoto and N. Scialabba, 1990. FAO species catalogue. Vol. 10. Gadiform fishes of the world (Order Gadiformes). An annotated and illustrated catalogue of cods, hakes, grenadiers and other gadiform fishes known to date. FAO Fish. Synop. 125(10). Rome: FAO. 442 p. 
enregistrées. Bulletin de la Société Scientifique de Bretagne 39:113-117.

Molva molva

Mora moro

Mugil cephalus

Mullus surmuletus
Pauly, D., 1978. A preliminary compilation of fish length growth parameters. Ber. Inst. Meereskd. Christian-Albrechts-Univ. Kiel (55):1-200

Amaral, V. and H.N. Cabral, 2004. Ecology of the whiskered sole in the Sado estuary, Portugal. J. Fish Biol. 64(2):460-474.

Vieira, A.R., Figueiredo, I., Figueiredo, C. and Menezes, G.M., 2013. Age and growth of two deep-water fish species in the Azores Archipelago: Mora moro (Risso, 1810) and Epigonus telescopus (Risso, 1810). Deep Sea Research Part II: Topical Studies in Oceanography, 98, pp.148-159.

Apostolidis, C. and K.I. Stergiou, 2014. Estimation of growth parameters from published data for several Mediterranean fishes. J. Appl. Ichthyol. 30(1):189-194.

Mahé, K., Coppin, F., Vaz, S. and Carpentier, A. 2013. Striped red mullet (M ullus surmuletus, L innaeus, 1758) in the eastern E nglish C hannel and southern $\mathrm{N}$ orth Sea: growth and reproductive biology. Journal of Applied Ichthyology, 29(5), pp.1067-1072.
Cohen, D.M., T. Inada, T. Iwamoto and N. Scialabba, 1990. FAO species catalogue. Vol. 10. Gadiform fishes of the world (Orde Gadiformes). An annotated and illustrated catalogue of cods, hakes, grenadiers and other gadiform fishes known to date. FAO Fish. Synop. 125(10). Rome: FAO. 442 p. Amaral, V. and H.N. Cabral, 2004. Ecology of the whiskered sole in the Sado estuary, Portugal. J. Fish Biol. 64(2):460-474.

Campillo, A., 1992. Les pêcheries françaises de Méditeranée: synthèse des

connaissances. Institut Francais de Recherche pour l'Exploitation de la Mer, France. $206 \mathrm{p}$ 
Mustelus mustelus/asterias

Farrell, E.D., S. Mariani and M.W. Clarke, 2010. Age and growth estimates for the starry smoothhound (Mustelus asterias) in the Northeast Atlantic Ocean. ICES J. Mar. Sci. 67:931-939.

Myctophum punctatum

Myliobatis aquila

Myoxocephalus quadricornis sculpin. Trudy Instituta Okeanologii AN SSSR 6:132-149.

Myoxocephalus scorpioides

Coad, B.W. and J.D. Reist, 2004. Annotated list of the arctic marine fishes of Canada. Can. MS Rep. Fish Aquat. Sci. 2674:iv:+112 p.

Myoxocephalus scorpius tropical Atlantic (CLOFETA). JNICT, Lisbon; SEI, Paris; and UNESCO, Paris. Vol. 1.

Luksenburg, J.A. and Pedersen, T., 2002. Sexual
Compagno, L.J.V., 1984. FAO Species Catalogue. Vol. 4. Sharks of the world. An annotated and illustrated catalogue of shark species known to date. Part 2 -

Carcharhiniformes. FAO Fish. Synop. 125(4/2):251-655. Rome: FAO.

Wörner, F., 1975. Untersuchungen an drei Myctophidenarten, Benthosema glaciale (Reinhardt, 1837), Ceratoscopelus

maderensis (Lowe, 1839) und Myctophum (M.) punctatum Rafinesque, 1810 aus dem Nordwestafrikanischen Auftriebgebieten, im Frühjahr 1972. Kiel University. 136 p. Thesis.

Breder, C.M. and D.E. Rosen, 1966. Modes of reproduction in fishes. T.F.H.

Publications, Neptune City, New Jersey. 941 p. and geographical variation in life history parameters of the shorthorn sculpin. Journal of fish biology, 61(6), pp.1453-1464.

Compagno, L.J.V., Dando, M. and Fowler, S.L. 2005. Sharks of the World. Harper Collins.
Luksenburg, J.A and Pedersen, T, 2002. Sexual and geographical variation in life history parameters of the shorthorn sculpin. Journal of fish biology, 61(6), pp.1453-1464 
Naucrates ductor

\section{Neoraja iberica}

Nerophis lumbriciformis

Nettastoma melanurum

Nezumia aequalis
Muus, B.J. and J.G. Nielsen, 1999. Sea fish. Scandinavian Fishing Year Book, Hedehusene, Denmark. 340 p.

Vassilopoulou, V., A. Siapatis, G. Christides and P. Bekas, 2004. The biology and ecology of juvenile pilotfish (Naucrates ductor) associated with Fish Aggregating Devices (FADs) in eastern Mediterranean waters. Medit. Mar. Sci. 5(1):5-17.

Stehmann, M.F.W., B. Séret, E.M. Costa and J. Baro, 2008. Neoraja iberica n. sp., a new species of pygmy skate (Elasmobranchii, Rajidae) from the southern slope of the Iberian Peninsula (Eastern North Atlantic). Cybium 32(1):51-71.

Dawson, C.E., 1986. Syngnathidae. p. 628-639. In P.J.P. Whitehead, M.-L. Bauchot, J.-C. Hureau, J. Nielsen and E. Tortonese (eds.) Fishes of the North-eastern Atlantic and the Mediterranean. Volume 2. Unesco, Paris.

Deval, M.C., Güven, O., Saygu, İ. and Kabapçioğlu, T., 2014. Length-weight relationships of 10 fish species found of Antalya Bay, eastern Mediterranean. Journal of applied ichthyology, 30(3), pp.567-568.

Massuti, E., B. Morales-Nin and C. Stefanescu, 1995. Distribution and biology of five grenadier
Muus, B.J. and J.G. Nielsen, 1999. Sea fish. Scandinavian Fishing Year Book, Hedehusene, Denmark. 340 p.

Stehmann, M.F.W., B. Séret, E.M. Costa and J. Baro, 2008. Neoraja iberica n. sp., a new species of pygmy skate (Elasmobranchii, Rajidae) from the southern slope of the Iberian Peninsula (Eastern North Atlantic). Cybium 32(1):51-71.

Porcu, C., Follesa, M.C., Gastoni, A., Mulas, A., Pedoni, C. and Cau, A., 2013. The reproductive cycle of a deep-sea eel, Nettastoma melanurum (Nettastomatidae: Anguilliformes) from the south-eastern Sardinian Sea (central-western Mediterranean). Journal of the Marine Biological Association of the United Kingdom, 93(4), pp.1105-1115.

Massuti, E., B. Morales-Nin and C. Stefanescu, 1995. Distribution and biology
Stehmann, M.F.W B Séret, E.M. Costa and J. Baro, 2008. Neoraja iberica n. sp. a new species of pygmy skate (Elasmobranchii, Rajidae) from the southern slope of the Iberian Peninsula (Eastern North Atlantic). Cybium 32(1):51-71. 
fish (Pisces: Macrouridae) from the upper and middle slope of the northwestern

Mediterranean. Deep-Sea Research 42(3):307330.

Nezumia sclerorhynchus

Rannou, M., 1976. Age et croissance d'un poisson bathyal: Nezumia sclerorhynchus (Macrouridae, Gadiformes) de la mer d'Alboran. Cah. Biol. Mar. 17(4):413-421.

Notoscopelus kröyer

Froese, R. and C. Binohlan, 2003. Simple methods to obtain preliminary growth estimates for fishes. J. Appl. Ichthyol. 19(6):376-379.

Ophidion barbatum

Pagellus acarne

Pagellus bogaraveo

Pagellus erythrinus

from the Canarian archipelago. J. Appl. Ichthyol. 16:41-47.

Lorance, P., 2010. History and dynamics of the overexploitation of the blackspot sea bream (Pagellus bogaraveo) in the Bay of Biscay. ICES Journal of Marine Science, 68(2), pp.290-301.

Coelho, R., Bentes, L., Correia, C., Gonçalves, J., of five grenadier fish (Pisces: Macrouridae) from the upper and middle slope of the northwestern Mediterranean. Deep-Sea Research 42(3):307-330.

Cohen, D.M., T. Inada, T. Iwamoto and N. Scialabba, 1990. FAO species catalogue. Vol. 10. Gadiform fishes of the world (Order Gadiformes). An annotated and illustrated catalogue of cods, hakes, grenadiers and other gadiform fishes known to date. FAO Fish. Synop. 125(10). Rome: FAO. 442 p.

Pajuelo, J.G. and J.M. Lorenzo, 2000.

Reproduction, age, growth and mortality of axillary seabream, Pagellus acarne

(Sparidae), from the Canarian archipelago.

J. Appl. Ichthyol. 16:41-47. Lino, P.G., Monteiro, P., Ribeiro, J. and Erzini, K., 2010. Life history of the common pandora,
Lorance, P., 2010. History and dynamics of the overexploitation of the blackspot sea bream (Pagellus bogaraveo) in the Bay of Biscay. ICES Journal of Marine Science, 68(2), pp.290-301.

Coelho, R., Bentes, L., Correia, C., Gonçalves, J., Lino, P.G., Monteiro, P., Ribeiro, J. and Erzini, K., 2010. Life history of the common pandora, Pagellus 
Pagellus erythrinus (Linnaeus,

1758)(Actinopterygii: Sparidae) from southern

Portugal. Brazilian Journal of Oceanography,

58(3), pp.233-245.

Pagrus pagrus

Pajuelo, J.G. and J.M. Lorenzo, 1996. Life history of the red porgy Pagrus pagrus

(Teleostei: Sparidae) off the Canary Islands, central east Atlantic. Fish. Res. 28(2):163-177.

Parablennius gattorugine

Bauchot, M.-L., 1987. Poissons osseux. p. 891-

1421. In W. Fischer, M.L. Bauchot and M.

Schneider (eds.) Fiches FAO d'identification

pour les besoins de la pêche. (rev. 1).

Méditerranée et mer Noire. Zone de pêche 37.

Vol. Il. Commission des Communautés

Européennes and FAO, Rome.

Paralichthys dentatus

Desfosse, J.C., 1995. Movements and ecology of summer flounder, Paralichthys dentatus, tagged in the southern mid-Atlantic bight. Unpubl. PhD. diss., College of William and Mary, Williamsburg, VA. 187 p.

Pegusa lascaris

Pholis gunnellus
Félix, P.M., Vinagre, C. and Cabral, H.N., 2011. Life-history traits of flatfish in the Northeast Atlantic and Mediterranean Sea. Journal of Applied Ichthyology, 27(1), pp.100-111.

Cheetham, C. and Fives, J.M., 1990, January. The biology and parasites of the butterfish Pholis gunnellus (Linnaeus, 1758) in the Galway Bay area. In Proceedings of the Royal Irish Academy. Section B: Biological, erythrinus (Linnaeus, 1758)(Actinopterygii: Sparidae) from southern Portugal. Brazilian Journal of Oceanography, 58(3), pp.233 245.

Pajuelo, J.G. and J.M. Lorenzo, 1996. Life history of the red porgy Pagrus pagrus

(Teleostei: Sparidae) off the Canary Islands, central east Atlantic. Fish. Res. 28(2):163177.

Desfosse, J.C., 1995. Movements and ecology of summer flounder, Paralichthys dentatus, tagged in the southern mid Atlantic bight. Unpubl. PhD. diss., College of William and Mary, Williamsburg, VA. 187 p.

Félix, P.M., Vinagre, C. and Cabral, H.N. 2011. Life-history traits of flatfish in the Northeast Atlantic and Mediterranean Sea. Journal of Applied Ichthyology, 27(1), pp.100-111.

Qasim, S.Z., 1957. The biology of

Centronotus gunnellus (L.)(Teleostei). The Journal of Animal Ecology, pp.389-401. 
Geological, and Chemical Science (pp. 127-

149). Royal Irish Academy.

Phrynorhombus norvegicus

Nielsen, J.G., 1986. Scophthalmidae. p. 1287-

1293. In P.J.P. Whitehead, M.-L. Bauchot, J.-C.

Hureau, J. Nielsen and E. Tortonese (eds.)

Fishes of the North-eastern Atlantic and the

Mediterranean. UNESCO, Paris. Vol. 3.

Phycis blennoides

Casas, J.M. and Piñeiro, C., 2000. Growth and age estimation of greater fork-beard (Phycis blennoides Brünnich, 1768) in the north and northwest of the Iberian Peninsula (ICES Division VIIIc and IXa). Fisheries Research, 47(1), pp.19-25.

Platichthys flesus

Pleuronectes platessa

Pollachius pollachius
Froese, R. and C.C. Frieß, 1992. Synopsis of biological data on Platichthys flesus (L.), ICES assessment units 22 and 24, using the FISHBASE format. ICES C.M.1992/J:41. International Council for the Exploration of the Sea, Copenhagen.

Van Walraven, L., Mollet, F.M., Van Damme, C.J.G. and Rijnsdorp, A.D., 2010. Fisheriesinduced evolution in growth, maturation and reproductive investment of the sexually dimorphic North Sea plaice (Pleuronectes platessa L.). Journal of Sea Research, 64(1-2), pp.85-93.

Pauly, D., 1978. A preliminary compilation of fish length growth parameters. Ber. Inst. Meereskd. Christian-Albrechts-Univ. Kiel (55):1-200.
Cohen, D.M., T. Inada, T. Iwamoto and N. Scialabba, 1990. FAO species catalogue. Vol. 10. Gadiform fishes of the world (Orde Gadiformes). An annotated and illustrated catalogue of cods, hakes, grenadiers and other gadiform fishes known to date. FAO Fish. Synop. 125(10). Rome: FAO. 442 p

ICES, 2014. Report of the Benchmark Workshop on Baltic Flatfish Stocks (WKBALFLAT), 27-31 January 2014, Copenhagen, Denmark. ICES CM 2014/ACOM:39. 320 pp.

Van Walraven, L., Mollet, F.M., Van Damme, C.J.G. and Rijnsdorp, A.D., 2010 Fisheries-induced evolution in growth, maturation and reproductive investment of the sexually dimorphic North Sea plaice (Pleuronectes platessa L.). Journal of Sea Research, 64(1-2), pp.85-93.

Alonso-Fernandez, A., Villegas-Rios, D. Valdés-López, M., Olveira-Dominguez, B. and Saborido-Rey, F., 2013. Reproductive biology of pollack (Pollachius pollachius) from the Galician shelf (north-west Spain).
Casas, J.M. and Piñeiro, C., 2000. Growth and age estimation of greater fork-beard (Phycis blennoides Brünnich, 1768) in the north and northwest of the Iberian Peninsula (ICES Division VIIIc and IXa). Fisheries Research, 47(1), pp.19-25. 
Pollachius virens

Polymetme corythaeola

Polymetme thaeocoryla

\section{Polyprion americanus}

Pomatoschistus lozanoi

Pomatoschistus norvegicus
Muus, B.J. and J.G. Nielsen, 1999. Sea fish.

Scandinavian Fishing Year Book, Hedehusene, Denmark. 340 p.

Beverton, R.J.H. and S.J. Holt, 1959. A review of the lifespans and mortality rates of fish in nature, and their relation to growth and other physiological characteristics. p. 142-180. In G.E.W. Wolstenholme and M. O'Connor (eds.) CIBA Foundation colloquia on ageing: the lifespan of animals. volume 5. J \& A Churchill Ltd, London.

Schaefer, S., R.K. Johnson and J. Badcock, 1986. Photichthyidae. p. 243-247. In M.M. Smith and P.C. Heemstra (eds.) Smiths' sea fishes. Springer-Verlag, Berlin.

Parin, N.V. and O.D. Borodulina, 1990. Survey of the genus Polymetme (Photichthyidae) with a description of two new species. J. Ichthyol. 30(6):108-121.

Sedberry et al. 1999 Wreckfish in the North Atlantic- Fisheries, Biology, and management of a widely distributed and Long-lived fish

Muus, B.J. and J.G. Nielsen, 1999. Sea fish.
Journal of the Marine Biological Association of the United Kingdom, 93(7), pp.1951-

1963.

Beverton, R.J.H. and S.J. Holt, 1959. A review of the lifespans and mortality rates of fish in nature, and their relation to growth and other physiological

characteristics. p. 142-180. In G.E.W. Wolstenholme and M. O'Connor (eds.) CIBA Foundation colloquia on ageing: the lifespan of animals. volume 5. J \& A Churchill Ltd, London.

Peres, M.B. and S. Klippel, 2003.

Reproductive biology of southwestern

Atlantic wreckfish, Polyprion americanus

(Teleostei: Polyprionidae). Environ. Biol. Fish. 68:163-173. 
Scandinavian Fishing Year Book, Hedehusene, Denmark. 340 p.

Prionace glauca

Pterycombus brama

Pungitius pungitius

Raja brachyura

Raja clavata

Raja microocellata
Nakano, Hideki, and John D. Stevens. 2009. "The Biology and Ecology of the Blue Shark, Prionace Glauca." Sharks of the Open Ocean: Biology, Fisheries and Conservation. wiley, 140-51. doi:10.1002/9781444302516.ch12.

Robins, C.R. and G.C. Ray, 1986. A field guide to Atlantic coast fishes of North America.

Houghton Mifflin Company, Boston, U.S.A. 354

Herczeg, G., Gonda, A., Kuparinen, A. and Merilä, J., 2012. Contrasting growth strategies of pond versus marine populations of ninespined stickleback (Pungitius pungitius): a combined effect of predation and competition?. Evolutionary Ecology, 26(1), pp.109-122.

Gallagher, M.J., Nolan, C.P. and Jeal, F., 2005. Age, growth and maturity of the commercial ray species from the Irish Sea. Journal of Northwest Atlantic Fishery Science, 35, pp.4766

Gallagher, M.J., Nolan, C.P. and Jeal, F., 2005 Age, growth and maturity of the commercial ray species from the Irish Sea. Journal of Northwest Atlantic Fishery Science, 35, pp.47 66

Ryland, J.S. and Ajayi, T.O. 1984. Growth and population dynamics of three Raja species in
Nakano, Hideki, and John D. Stevens. 2009. "The Biology and Ecology of the Blue Shark, Prionace Glauca." Sharks of the Open

Ocean: Biology, Fisheries and Conservation. wiley, $140-51$.

doi:10.1002/9781444302516.ch12.

Herczeg, G., Gonda, A., Kuparinen, A. and Merilä, J., 2012. Contrasting growth strategies of pond versus marine populations of nine-spined stickleback (Pungitius pungitius): a combined effect of predation and competition?. Evolutionary Ecology, 26(1), pp.109-122.

Gallagher, M.J., Nolan, C.P. and Jeal, F. 2005. Age, growth and maturity of the commercial ray species from the Irish Sea. Journal of Northwest Atlantic Fishery Science, 35, pp.47-67

Gallagher, M.J., Nolan, C.P. and Jeal, F., 2005. Age, growth and maturity of the commercial ray species from the Irish Sea. Journal of Northwest Atlantic Fishery Science, 35, pp.47-67

McCully, S.R., F. Scott and J.R. Ellis, 2012. Lengths at maturity and conversion factors
Stevens, J.D., 1975. Vertebral rings as a means of age determination in the blue shark (Prionace glauca L.). J. Mar. Biol. Ass. U.K. 55:657-665.

Clark, R.S., 1922. Rays and Skates (Raiœ) No. 1.-Egg-Capsules and Young. Journal of the Marine Biological Association of the United Kingdom, 12(4), pp.578-643

Pawson, M.G. and J.R. Ellis, 2005. Stock identity of elasmobranchs in the northeast Atlantic in relation to assessment and management. J. Northw. Atl. Fish. Sci., 35: 173-193.

Clark, R.S., 1922. Rays and Skates (Raiœ) No. 1.-Egg-Capsules and Young. Journal 
Carmarthen Bay, British Isles. Journal de Conseil Internationale de Exploration de la Mer 41: 111-120.

Raja montagui

Gallagher, M.J., C.P. Nolan and F. Jeal, 2005. Age, growth and maturity of the commercial ray species from the Irish Sea. J. Northw. Atl. Fish. Sci., 35: 47-66.

Moura, T., Figueiredo, I., Farias, I., SerraPereira, B., Coelho, R., Erzini, K., Neves, A. and Gordo, L.S., 2007. The use of caudal thorns for ageing Raja undulata from the Portuguese continental shelf, with comments on its reproductive cycle. Marine and Freshwater Research, 58(11), pp.983-992.

Rajella bathyphila

Ebert \& Stehmann 2013 Sharks, batoids and chimaeras of the North Atlantic. FAO Species Catalogue for Fishery Purposes (7) Rome, 523 pp.

Rajella fyllae

Rajella lintea

Raniceps raninus

Muus, B.J. and J.G. Nielsen, 1999. Sea fish. Scandinavian Fishing Year Book, Hedehusene, Denmark. 340 p.

Ponomarenko, N.P. 1961. Raja lintea Fries at the border between the Norwegian and the Barents Sea. [in Russian]. Zoologichesky Zhurnal 40(8): 1260-1261.

Cohen, D.M., T. Inada, T. Iwamoto and N Scialabba, 1990. FAO species catalogue. Vol. for skates (Rajidae) around the British Isles, with an analysis of data in the literature. ICES J. Mar. Sci. 69(10):1812-1822.

Gallagher, M.J., C.P. Nolan and F. Jeal, 2005. Age, growth and maturity of the commercial ray species from the Irish Sea. J. Northw. Atl. Fish. Sci., 35: 47-66.

Serra-Pereira, B., Erzini, K. and Figueiredo, I., 2015. Using biological variables and reproductive strategy of the undulate ray Raja undulata to evaluate productivity and susceptibility to exploitation. Journal of fish biology, 86(5), pp.1471-1490.

of the Marine Biological Association of the United Kingdom, 12(4), pp.578-643

Serena, F., 2005. Field identification guide to the sharks and rays of the Mediterranean and Black Sea. Rome : Food and Agriculture Organization of the United Nations; Ix, 97 p., [14] leaves of plates :ill. (some col.), col. Maps ; $25 \mathrm{~cm}$

Serra-Pereira, B., Erzini, K. and Figueiredo, I., 2015. Using biological variables and reproductive strategy of the undulate ray Raja undulata to evaluate productivity and susceptibility to exploitation. Journal of fish biology, 86(5), pp.1471-1490.

Clark, R.S., 1922. Rays and Skates (Raiœ) No. 1.-Egg-Capsules and Young. Journal of the Marine Biological Association of the United Kingdom, 12(4), pp.578-643

Bigelow, H.B. and W.C. Schroeder, 1953. Sawfishes, guitarfishes, skates and rays. p. 1-514. In J. Tee-Van et al. (eds.) Fishes of the western North Atlantic. Part two. New Haven, Sears Found. Mar. Res., Yale Univ. 
10. Gadiform fishes of the world (Order Gadiformes). An annotated and illustrated catalogue of cods, hakes, grenadiers and other gadiform fishes known to date. FAO Fish.

Synop. 125(10). Rome: FAO. 442 p.

Rostroraja alba

Kadri, H., Marouani, S., Bradai, M.N., Bouaïn, A., \& Morize, E. (2014). Age, Growth,

Mortality, Longevity and Reproductive Biology of the White Skate, Rostroraja alba

(Chondrichthyes: Rajidae) of the Gulf of Gabès (Southern Tunisia, Central Mediterranean).

Turkish Journal of Fisheries and Aquatic

Sciences, 14, 193-204.

http://doi.org/10.4194/1303-2712-v14_1_21

Sarda sarda

Rey, J.C., E. Alot and A. Ramos, 1986. Growth of the Atlantic bonito (Sarda sarda Bloch,

1793) in the Atlantic and Mediterranean area of the Strait of Gibraltar. Inv. Pesq. 50(2):179 185.

Sardina pilchardus

Pauly, D., 1978. A preliminary compilation of fish length growth parameters. Ber. Inst. Meereskd. Christian-Albrechts-Univ. Kiel (55):1-200.

Sarpa salpa
Villamil, M.M., Lorenzo, J.M., Pajuelo, J.G. Ramos, A. and Coca, J., 2002. Aspects of the life history of the salema, Sarpa salpa (Pisces, Sparidae), off the Canarian Archipelago (central-east Atlantic). Environmental Biology of Fishes, 63(2), pp.183-192.
Kadri, H., Marouani, S., Bradai, M.N., Bouaïn, A., \& Morize, E. (2014). Age,

Growth, Mortality, Longevity and

Reproductive Biology of the White Skate, Rostroraja alba (Chondrichthyes: Rajidae) of the Gulf of Gabès (Southern Tunisia, Central Mediterranean). Turkish Journal of Fisheries and Aquatic Sciences, 14, 193204. http://doi.org/10.4194/1303-2712v14_1_21

Cayré, P., J.B. Amon Kothias, T. Diouf and J.M. Stretta, 1993. Biology of tuna. p. 147244. In A. Fonteneau and J. Marcille (eds.) Resources, fishing and biology of the tropical tunas of the Eastern Central Atlantic. FAO Fish. Tech. Pap. 292. Rome, FAO. $354 \mathrm{p}$

Beverton, R.J.H., 1963. Maturation, growth and mortality of clupeid and engraulid stocks in relation to fishing. Cons. Perm. Int. Explor. Mer, Rapp. p.-v. Réun. 154:44-67.

Villamil, M.M., Lorenzo, J.M., Pajuelo, J.G., Ramos, A. and Coca, J., 2002. Aspects of the life history of the salema, Sarpa salpa (Pisces, Sparidae), off the Canarian Archipelago (central-east Atlantic).

Environmental Biology of Fishes, 63(2) pp.183-192. 
Scomber colias

Scomberesox saurus

Scophthalmus maximus

Scophthalmus rhombus

Scorpaena loppe
Vasconcelos, J.M.A., M.A. Dias and G. Faria, 2011. Age and growth of the Atlantic chub mackerel Scomber colias Gmelin, 1789 off Madeira Island. Arquipelago. Life Mar. Sci. 28:57-70.

Villamor, B., Abaunza, P. and Fariña, A.C. 2004. Growth variability of mackerel (Scomber scombrus) off north and northwest Spain and a comparative review of the growth patterns in the northeast Atlantic. Fisheries Research, 69(1), pp.107-121.

Potoschi, A., 1996. Observations about some biological aspects of Scomberesox saurus (Walbaum, 1792) in the area of the Straits of Messina. Oebalia 12:139-146.

Jones, A., 1974. Sexual maturity, fecundity and growth of the turbot, Scophthalmus maximus L. J. Mar. Biol. Ass. U.K. 54:109-125.

Félix, P.M., Vinagre, C. and Cabral, H.N., 2011. Life-history traits of flatfish in the Northeast Atlantic and Mediterranean Sea. Journal of Applied Ichthyology, 27(1), pp.100-111.

Ordines, F., M. Valls and A. Gouraguine, 2012. Biology, feeding, and habitat preferences of Cadenat's rockfish, Scorpaena loppei (Actinopterygii: Scorpaeniformes:

Scorpaenidae), in the Balearic Islands (western Mediterranean). Acta Ichthyol. Piscat.

42(1):21-30.
Vasconcelos, J., M. Afonso-Dias and G. Faria, 2012. Atlantic chub mackerel (Scomber colias) spawning season, size and age at maturity in Madeira waters. Arquipelago. Life Mar. Sci. 29:43-51.
Jones, A., 1974. Sexual maturity, fecundity and growth of the turbot, Scophthalmus maximus L. J. Mar. Biol. Ass. U.K. 54:109125.

Félix, P.M., Vinagre, C. and Cabral, H.N 2011. Life-history traits of flatfish in the Northeast Atlantic and Mediterranean Sea. Journal of Applied Ichthyology, 27(1), pp.100-111. 
Neves, A., V. Sequeira, A.R. Vieira, R.B. Paiva and L.S. Gordo, 2015. Age and growth of small red scorpionfish, Scorpaena notata

(Actinopterygii: Scorpaeniformes; Scorpaenidae), a common discard species from the Portuguese fishery. Acta Ichthyol. Piscat. 45(1):13-20.

\section{Scorpaena porcus}

Scorpaena scrofa

Scyliorhinus canicula

Scyliorhinus stellaris

Scymnodon ringens
Siblot-Bouteflika, D., 1976. Contribution à l'étude des Scorpènidés de la région d'Alger. University de Marseille. Thesis.

Matić-Skoko, S., Stagličić, N., Kraljević, M., Pallaoro, A. and Dulčić, J., 2015. The biological traits of the large red scorpionfish, Scorpaena scrofa: Temporal and ontogenetic dynamics. Estuarine, Coastal and Shelf Science, 152, pp.91-99.

Ivory, P., F. Jeal and C.P. Nolan, 2005. Age determination, growth and reproduction in the lesser-spotted dogfish, Scyliorhinus canicula (L.). J. Northw. Atl. Fish. Sci. 35:89-106.

Ellis, J.R., Dulvy, N.K., Jennings, S., ParkerHumphreys, M. and Rogers, S.I., 2005.

Assessing the status of demersal

elasmobranchs in UK waters: a review. JMBAJournal of the Marine Biological Association of the United Kingdom, 85(5), pp.1025-1047.

Compagno, L.J.V., 1984. FAO Species Catalogue. Vol. 4. Sharks of the world. An annotated and illustrated catalogue of shark
Bradai, M N and A. Bouain, 1990.

Données sur la reproduction de Scorpaena porcus du Golfe de Gabès. Rapp. Comm. Int. Mer Médit. 32(1):265.

Matić-Skoko, S., Stagličić, N., Kraljević, M. Pallaoro, A. and Dulčić, J., 2015. The biological traits of the large red scorpionfish, Scorpaena scrofa: Temporal and ontogenetic dynamics. Estuarine Coastal and Shelf Science, 152, pp.91-99.

Ivory, P., F. Jeal and C.P. Nolan, 2005. Age determination, growth and reproduction in the lesser-spotted dogfish, Scyliorhinus canicula (L.). J. Northw. Atl. Fish. Sci. 35:89 106.

Ivory, P., F. Jeal and C.P. Nolan, 2005. Age determination, growth and reproduction in the lesser-spotted dogfish, Scyliorhinus canicula (L.). J. Northw. Atl. Fish. Sci. 35:89-106.

Ellis, J.R., Dulvy, N.K., Jennings, S., Parker-Humphreys, M. and Rogers, S.I. 2005. Assessing the status of demersal elasmobranchs in UK waters: a review. JMBA-Journal of the Marine Biological Association of the United Kingdom, 85(5), pp.1025-1047. 
species known to date. Part 1 -

Hexanchiformes to Lamniformes. FAO Fish.

Synop. 125(4/1):1-249. Rome, FAO.

Sebastes viviparus

Serranus cabrilla

Serranus scriba

Solea senegalensis
Jennings, S., S.P.R. Greenstreet and J.D. Reynolds, 1999. Structural change in an exploited fish community: a consequence of differential fishing effects on species with contrasting life histories. J. Animal Ecol. 68:617-627.

Bouain, A., 1983. Croissance lineaire des serrans des cotes sud-est de la tunisie. Rapp. Comm. int. Mer Médit., 28(5):87-91.

Labropoulou M., Tserpes G., Tsimenidis N. 1998. Age, growth and feeding habits of the brown comber Serranus hepatus (Linnaeus, 1758) on the Cretan shelf. Estuarine Coast. Shelf Science, 46: 723-732.

Bouain, A., 1983. Croissance lineaire des serrans des cotes sud-est de la tunisie. Rapp. Comm. int. Mer Médit., 28(5):87-91.

Félix, P.M., C. Vinagre and H.N Cabral, 2011. Life-history traits of flatfish in the Northeast Atlantic and Mediterranean Sea. J. Appl. Ichthyol. 27:100-111.
Drevetnyak, K.V. and Kluev, A.I, 2005.

Advances in Reproductive Biology:

Methodology and Applications for Fisheries

Science (Session Q). ICES CM 2005/Q:31

García-Díaz, M.M., V.M. Tuset, J.A.

González and J. Socorro, 1997. Sex and

reproductive aspects in Serranus cabrilla (Osteichthyes: Serranidae): macroscopic and histological approaches. Mar. Biol.

127:379-386.

Soykan, O., A.T. Ilkyaz, G. Metin and H.T.

Kinacigil, 2013. Growth and reproduction of brown comber (Serranus hepatus Linnaeus,

1758 ) in the central Aegean Sea, Turkey.

Turk. J. Zool. 37:211-217.

Tuset, V.M., M.M. García-Díaz, J.A. González, M.J. Lorente and I.J. Lozano,

2005. Reproduction and growth of the painted comber Serranus scriba

(Serranidae) of the marine reserve of Lanzarote Island (central-eastern Atlantic). Estuar. Coast. Shelf Sci. 64(2-3):335-346. 
Sparus aurata

Spondyliosoma cantharus

Sprattus sprattus
Du Pontavice, H., Randon, M., Lehuta, S., Vermard, Y. and Savina-Rolland, M., 2018. Investigating spatial heterogeneity of von Bertalanffy growth parameters to inform the stock structuration of common sole, Solea solea, in the Eastern English Channel. Fisheries research, 207, pp.28-36.

Nielsen, J., Hedeholm, R.B., Heinemeier, J., Bushnell, P.G., Christiansen, J.S., Olsen, J.,

Ramsey, C.B., Brill, R.W., Simon, M., Steffensen, K.F. and Steffensen, J.F., 2016. Eye lens radiocarbon reveals centuries of longevity in the Greenland shark (Somniosus microcephalus). Science, 353(6300), pp.702704.

Arias, A., 1980. Crecimento, régimen, alimentarión y reproduc-ción de la dorada (Sparus aurataL.) y del róbalo(Dicentrarchus labraxL.) en los esteros de Cádiz. Inv. Pesq. 44: 59-83.

Neves, A., Vieira, A.R., Sequeira, V., Paiva, R.B. and Gordo, L.S., 2017. Modelling the growth of a protogynous sparid species, Spondyliosoma cantharus (Teleostei: Sparidae). Hydrobiologia, 797(1), pp.265-275

Iles, T.D. and P.O. Johnson, 1962. The correlation table analysis of a sprat (Clupea sprattus L.) year-class to separate two groups differing in growth characteristics. J. du Conseil 27(1):287-303.
Mollet, F.M., Kraak, S.B. and Rijnsdorp, A.D., 2007. Fisheries-induced evolutionary changes in maturation reaction norms in North Sea sole Solea solea. Marine Ecology Progress Series, 351, pp.189-199.

Yano, K., Stevens, J.D. and Compagno, L.J.V., 2007. Distribution, reproduction and feeding of the Greenland shark Somniosus (Somniosus) microcephalus, with notes on two other sleeper sharks, Somniosus (Somniosus) pacificus and Somniosus (Somniosus) antarcticus. Journal of fish biology, 70(2), pp.374-390.

Bauchot, M.-L. and J.-C. Hureau, 1986. Sparidae. p. 883-907. In P.J.P. Whitehead M.-L. Bauchot, J.-C. Hureau, J. Nielsen and E. Tortonese (eds.) Fishes of the northeastern Atlantic and the Mediterranean. volume 2. UNESCO, Paris.

Gonçalves, J.M. and Erzini, K.A.R.I.M., 2000. The reproductive biology of Spondyliosoma cantharus (L.) from the SW Coast of Portugal. Scientia Marina, 64(4), pp.403411.
Yano, K., Stevens, J.D. and Compagno, L.J.V., 2007. Distribution, reproduction and feeding of the Greenland shark Somniosus (Somniosus) microcephalus, with notes on two other sleeper sharks, Somniosus (Somniosus) pacificus and Somniosus (Somniosus) antarcticus. Journal of fish biology, 70(2), pp.374390. 
Squalus acanthias

Stomias boa

Symphodus bailloni

Symphodus melops

Synaphobranchus kaupi
Slauson, T.P., P.M.J. Woodhead and R. Castaneda, 1983. The age, growth and maturation of the spiny dogfish Squalus acanthias, in the Northwest Atlantic. Mar. Sci. Res. Cent. Spec. Rept. No. 49, State Univ. Stony Brook, N. Y., USA

Gibbs, R.H. Jr., 1990. Stomiidae. p. 296-299. In J.C. Quero, J.C. Hureau, C. Karrer, A. Post and L. Saldanha (eds.) Check-list of the fishes of the eastern tropical Atlantic (CLOFETA). JNICT, Lisbon; SEI, Paris; and UNECO, Paris. Vol. 1.

Quignard, J.-P. and A. Pras, 1986. Labridae. p. 919-942. In P.J.P. Whitehead, M.-L. Bauchot, J.C. Hureau, J. Nielsen and E. Tortonese (eds.) Fishes of the north-eastern Atlantic and the Mediterranean. UNESCO, Paris. Vol. 2.

Pauly, D., 1978. A preliminary compilation of fish length growth parameters. Ber. Inst. Meereskd. Christian-Albrechts-Univ. Kiel (55):1-200.

Quignard, J.-P., 1966. Recherches sur les Labridae (Poissons Téléostéens) perciformes des côtes européennes. Systématique et biologie. Naturalia Monspeliensia Sér. Zoologique 5:1-247.

Reiner, F., 1996. Catálogo dos peixes do arquipélago de Cabo Verde. Publ. Avuls. Inst Port. Invest. Mar. 2:339 p. (cited in Fishbase)
Slauson, T.P., P.M.J. Woodhead and R. Castaneda, 1983. The age, growth and maturation of the spiny dogfish Squalus acanthias, in the Northwest Atlantic. Mar.

Sci. Res. Cent. Spec. Rept. No. 49, State Univ. Stony Brook, N. Y., USA

Quignard, J.-P. and A. Pras, 1986. Labridae. p. 919-942. In P.J.P. Whitehead, M.-L. Bauchot, J.-C. Hureau, J. Nielsen and E.

Tortonese (eds.) Fishes of the north-eastern Atlantic and the Mediterranean. UNESCO, Paris. Vol. 2.

Quignard, J.-P. and A. Pras, 1986. Labridae. p. 919-942. In P.J.P. Whitehead, M.-L.

Bauchot, J.-C. Hureau, J. Nielsen and E.

Tortonese (eds.) Fishes of the north-eastern Atlantic and the Mediterranean. UNESCO, Paris. Vol. 2.
Holden, M. J., and Meadows, P.S. 1962. 1964. The fecundity of the spurdog (Squalus acanthias L.). ICES J. Con., 28: 418-424. 
Taurulus bubalis

Thunnus thynnus

Torpedo marmorata

Torpedo nobiliana
Lamp, F., 1965. Beiträge zur Bestandskunde und Fortpflanzungsbiologie der Seeskorpione, Myoxocephalus scorpius (Linnaeus 1758) und Taurulus bubalis (Euphrasen 1786) in der Kieler Förde. Kiel University. 140 p. Thesis.

Fedorov, V.V., 1986. Cottidae. p. 1243-1260. In P.J.P. Whitehead, M.-L. Bauchot, J.-C. Hureau, J. Nielsen and E. Tortonese (eds.) Fishes of the North-eastern Atlantic and the Mediterranean. UNESCO, Paris. Vol. 3.

Cort, J.L., 1991. Age and growth of the bluefin tuna, Thunnus thynnus (L.) of the Northeast Atlantic. ICCAT/SCRS/90/66:213-230.

Duman, Ö.V., \& Başusta, N. (2013). Age and Growth Characteristics of Marbled Electric Ray Torpedo marmorata (Risso, 1810) Inhabiting Iskenderun Bay, North-eastern Mediterranean Sea. Turkish Journal of Fisheries and Aquatic Sciences, 13, 541-549.

http://doi.org/10.4194/1303-2712-v13_3_19

McEachran, J.D. and de Carvalho, M.R. 2002. Batoid fishes. In: In: Carpenter, K.E. (ed). (ed.), The Living Marine Resources of the Western Central Atlantic. Volume 1. Introduction, molluscs, crustaceans, hagfishes, sharks, batoid fishes and chimaeras., pp. Pp. 508-589. FAO, Rome.
Corriero, A., Karakulak, S., Santamaria, N. Deflorio, M., Spedicato, D., Addis, P., Desantis, S., Cirillo, F., Fenech-Farrugia, A. Vassallo-Agius, R. and De la Serna, J.M. 2005. Size and age at sexual maturity of female bluefin tuna (Thunnus thynnus $L$.

1758) from the Mediterranean Sea. Journal of Applied Ichthyology, 21(6), pp.483-486. Møller, P.R., 1995. Electric fishes: history and behavior. Chapman \& Hall, London. $584 \mathrm{p}$.

Last, P.R., W.T. White, M.R. de Carvalho, B. Séret, M.F.W. Stehmann and G.J.P. Naylor, 2016. Rays of the world. CSIRO Publishing, Comstock Publishing Associates. i-ix + 1-790

Stehmann, M. and D.L. Bürkel, 1984 Torpedinidae. p. 159-162. In P.J.P. Whitehead, M.-L. Bauchot, J.-C. Hureau, J. Nielsen and E. Tortonese (eds.) Fishes of the north-eastern Atlantic and Mediterranean. UNESCO, Paris. Vol. 1. 
Trachinus draco

Bagge, O., 2004. The biology of the greater weever (Trachinus draco) in the commercial fishery of the Kattegat. ICES Journal of Marine Science, 61(6), pp.933-943.

Trachipterus arcticus

Froese, R. and C. Binohlan, 2003. Simple methods to obtain preliminary growth estimates for fishes. J. Appl. Ichthyol. 19(6):376-379.

Trachurus mediterraneus

Zupa, R., T. Silecchia, P. Carbonara and M.T. Spedicato, 2006. Growth of Trachurus mediterraneus (Steindachner, 1868) in the central-southern Tyyrhenian Sea. Biol. Mar. Medit. 13(2):302-303.

Trachurus picturatus

Vasconcelos, J., A. Alves, E. Gouveia and G. Faria, 2006. Age and growth of the blue jack mackerel, Trachurus picturatus Bowdich, 1825 (Pisces: Teleostei) off the Madeira archipelago. Arquipélago. Life Mar. Sci. 23A:47-57.

Trachurus trachurus

Trachyrincus murrayi
Abaunza, P., Gordo, L., Karlou-Riga, C., Murta, A., Eltink, A.T.G.W., Santamaría, M.G.,

Zimmermann, C., Hammer, C., Lucio, P., Iversen, S.A. and Molloy, J., 2003. Growth and reproduction of horse mackerel, Trachurus trachurus (Carangidae). Reviews in Fish Biology

ICES, 2016. Report of the Working Group on Biology and Assessment of Deep-sea Fisheries Resources (WGDEEP), 20-27 April 2016, ICES and Fisheries, 13(1), pp.27-61.
Stergiou, K.I., E.D. Christou, D.

Georgopoulous, A. Zenetos and C.

Souvermezoglou, 1997. The Hellenic seas: physics, chemistry, biology and fisheries. $p$. 415-538. In A.D. Ansell, R.N. Gibson and M. Barnes (eds.). Oceanography and marine biology: an annual review. UCL Press. Garcia, A., Canha, Â., Reis, D. and Diogo, H. 2015. Life history parameters of blue jack mackerel Trachurus picturatus (Teleostei Carangidae) from north-east Atlantic.

Journal of the Marine Biological Association of the United Kingdom, 95(2), pp.401-410.

Abaunza, P., Gordo, L., Karlou-Riga, C., Murta, A., Eltink, A.T.G.W., Santamaría,

M.G., Zimmermann, C., Hammer, C., Lucio, P., Iversen, S.A. and Molloy, J., 2003.

Growth and reproduction of horse mackerel, Trachurus trachurus

(Carangidae). Reviews in Fish Biology and Fisheries, 13(1), pp.27-61. 
HQ, Copenhagen, Denmark. ICES CM 2016/ACOM:18. 648 pp.

Trachyrincus scabrus

Trigla lyra

Trigloporus lastoviza

Triglops murrayi

Trisopterus esmarkii

Trisopterus luscus
Motais, R., 1960. Quelques observations sur la biologie d'un poisson abyssal, Trachyrinchus trachyrinchus Risso, et sur les conditions de vie en mer profonde. Bull. Inst. Océanogr. Monaco (1165):79 p.

Campillo, A., 1992. Les pêcheries françaises de Méditeranée: synthèse des connaissances.

Institut Francais de Recherche pour

I'Exploitation de la Mer, France. 206 p.

Ordines, F., Farriols, M.T., Lleonart, J., Guijarro, B., Quetglas, A. and Massutí, E., 2014. Biology and population dynamics of by-catch fish species of the bottom trawl fishery in the western Mediterranean. Mediterranean Marine Science, 15(3), pp.613-625.

Ottesen, C., 2004. Taxonomy, morphology and biology of Triglops murrayi and Triglops nybelini (family Cottidae) obtained at Svalbard and Jan Mayen. MS thesis, University of Troms $\varnothing$, Troms $\varnothing$, Norway .

Lambert, G., Nielsen, J.R., Larsen, L.I. and Sparholt, H., 2009. Maturity and growth population dynamics of Norway pout

(Trisopterus esmarkii) in the North Sea,

Skagerrak, and Kattegat. ICES Journal of Marine Science, 66(9), pp.1899-1914.

Labarta, U. and M.J. Ferreiro, 1982. Age and growth of the Galician coast pouting
Campillo, A., 1992. Les pêcheries françaises de Méditeranée: synthèse des

connaissances. Institut Francais de

Recherche pour l'Exploitation de la Mer, France. $206 \mathrm{p}$

Ordines, F., Farriols, M.T., Lleonart, J., Guijarro, B., Quetglas, A. and Massutí, E. 2014. Biology and population dynamics of by-catch fish species of the bottom trawl fishery in the western Mediterranean. Mediterranean Marine Science, 15(3), pp.613-625

Lambert, G., Nielsen, J.R., Larsen, L.I. and Sparholt, H., 2009. Maturity and growth population dynamics of Norway pout

(Trisopterus esmarkii) in the North Sea, Skagerrak, and Kattegat. ICES Journal of Marine Science, 66(9), pp.1899-1914.

Labarta, U. and M.J. Ferreiro, 1982. Age and growth of the Galician coast pouting 
(Trisopterus luscus L.). Preliminary data. ICES C.M. 1982/G:65.

Trisopterus minutus

Umbrina canariensis

Xenodermichthys copei

Xiphias gladius

Zeugopterus punctatus
Beverton, R.J.H. and S.J. Holt, 1959. A review of the lifespans and mortality rates of fish in nature, and their relation to growth and other physiological characteristics. p. 142-180. In G.E.W. Wolstenholme and M. O'Connor (eds.) CIBA Foundation colloquia on ageing: the lifespan of animals. volume 5. J \& A Churchill Ltd, London.

Dardignac, J., 1961. Les ombrines de côtes Atlantique du Maroc. Rev. Trav. Inst. Pêches. Marit. 25(3):263-279.

Bernardes, R.Á., J.L. de Figueiredo, A.R. Rodrigues, L.G. Fischer, C.M. Vooren, M. 2005. Peixes de zona econômica exclusiva da região sudeste-sul do Brasil: Levantamento com armadilhas, pargueiras e rede de arrasto de fundo. São Paulo: Editora da Universidade de São Paulo. 295 p.

Valeiras, X., J.M. de la Serna, D. Macías, M. Ruiz, S. García-Barcelona, M.J. Gómez and J.M Ortíz de Urbina, 2008. Age and growth of swordfish (Xiphias gladius) in the western Mediterranean Sea. Collect. Vol. Sci. Pap. ICCAT 62(4):1112-1121.

Nielsen, J.G., 1986. Scophthalmidae. p. 1287 1293. In P.J.P. Whitehead, M.-L. Bauchot, J.-C. Hureau, J. Nielsen and E. Tortonese (eds.)
Haimovici and C.L.D.B. Rossi-Wongtschowski,
(Trisopterus luscus L.). Preliminary data ICES C.M. 1982/G:65.

Beverton, R.J.H. and S.J. Holt, 1959. A review of the lifespans and mortality rates of fish in nature, and their relation to growth and other physiological

characteristics. p. 142-180. In G.E.W

Wolstenholme and M. O'Connor (eds.) CIBA

Foundation colloquia on ageing: the lifespan of animals. volume 5 . J \& A Churchill Ltd, London.
Macías, D., Hattour, A., De la Serna, J.M., Gómez-Vives, M.J. and Godoy, D., 2005.

Reproductive characteristics of swordfish (Xiphias gladius) caught in the

southwestern Mediterranean during 2003.

Col. Vol. Sci. Pap. ICCAT, 58(2), pp.454-469. 
Fishes of the North-eastern Atlantic and the Mediterranean. UNESCO, Paris. Vol. 3.

Zeugopterus regius

Zeus faber

Zoarces viviparus
Bauchot, M.-L., 1987. Poissons osseux. p. 8911421. In W. Fischer, M.L. Bauchot and M.

Schneider (eds.) Fiches FAO d'identification pour les besoins de la pêche. (rev. 1).

Méditerranée et mer Noire. Zone de pêche 37.

Vol. II. Commission des Communautés

Européennes and FAO, Rome.

Dunn, M.R., 2001. The biology and exploitation of John dory, Zeus faber (Linnaeus, 1758) in the waters of England and Wales. ICES Journal of Marine Science, 58(1), pp.96-105.

Wiêcaszek, B., 1998. On the morphometry and growth rate in the viviparous eel-pout, Zoarces viviparus (Zoarcidae) from waters of different salinity. Ital. J. Zool. 65:211-214.
Dunn, M.R., 2001. The biology and exploitation of John dory, Zeus faber (Linnaeus, 1758) in the waters of England and Wales. ICES Journal of Marine Science, 58(1), pp.96-105.

Muus, B.J. and J.G. Nielsen, 1999. Sea fish Scandinavian Fishing Year Book,

Hedehusene, Denmark. 340 p.
Muus, B.J. and J.G. Nielsen, 1999. Sea fish. Scandinavian Fishing Year Book Hedehusene, Denmark. 340 p. 
Table S4. Years included for each survey.

\begin{tabular}{|c|c|c|}
\hline Region & Survey & Years included \\
\hline \multirow[t]{2}{*}{ Baltic Sea } & BITS Q1 small & $1996-2018$ \\
\hline & BITS Q4 small & 1999-2018 \\
\hline \multirow[t]{5}{*}{ North Sea } & BTS & $1987-2018$ \\
\hline & DYFS & $2002-2018$ \\
\hline & IBTS Q1 & $1967-2018$ \\
\hline & IBTS Q3 & 1991-2018 \\
\hline & SNS & $2002-2018$ \\
\hline \multirow[t]{6}{*}{ West of UK } & ROCKALL & $1999,2001-2003,2005-2009,2011-2018$ \\
\hline & SWC-IBTS Q1 & $1985-2018$ \\
\hline & SWC-IBTS Q4 & 1990-2009, 2011-2018 \\
\hline & IE-IGFS & $2011-2018$ \\
\hline & NIGFS & $2006-2017$ \\
\hline & SP-PORC & $2001-2018$ \\
\hline \multirow[t]{4}{*}{ Bay of Biscay/Celtic Sea } & BTSVIII & 2011-2017 \\
\hline & EVHOE & 1997-2016 \\
\hline & FR-CGFS & $1988-2018$ \\
\hline & SP-NORTH & 1990-1992, 1994, 1997, 2001-2018 \\
\hline \multirow[t]{2}{*}{ Iberian Peninsula } & PT-IBTS & 2002-2011, 2013-2017 \\
\hline & SP-ARSA & $1996,2000-2018$ \\
\hline
\end{tabular}


Table S5. $F_{25 \% S S B}$ average and range for each IUCN category.

\begin{tabular}{lcccc}
\hline IUCN & Average & Minimum & Maximum & Number of \\
rating & & & & species \\
\hline CR & 0.17 & 0.05 & 0.39 & 3 \\
\hline EN & 0.37 & 0.20 & 0.75 & 7 \\
\hline VU & 0.27 & 0.13 & 0.46 & 8 \\
\hline NT & 1.54 & 0.09 & 20 & 18 \\
\hline LC & 9.96 & 0.10 & 20 & 207 \\
\hline DD & 6.17 & 0.28 & 20 & 19 \\
\hline
\end{tabular}


Table S6. 2019 EU Catch and landing obligations.

\begin{tabular}{|c|c|c|}
\hline $\begin{array}{l}\text { Type of } \\
\text { management }\end{array}$ & Species & Management applicable \\
\hline \multirow[t]{14}{*}{ No TAC } & Anarhichas minor & No TAC \\
\hline & Anarhichas lupus & No TAC \\
\hline & $\begin{array}{l}\text { Argyrosomus } \\
\text { regius }\end{array}$ & No TAC \\
\hline & Brama brama & No TAC \\
\hline & $\begin{array}{l}\text { Chimaera } \\
\text { monstrosa }\end{array}$ & No TAC \\
\hline & Conger conger & No TAC \\
\hline & Dasyatis pastinaca & No TAC \\
\hline & $\begin{array}{l}\text { Dasyatis } \\
\text { tortonesei }\end{array}$ & No TAC \\
\hline & $\begin{array}{l}\text { Dicentrarchus } \\
\text { punctatus }\end{array}$ & No TAC \\
\hline & Ephippion guttifer & No TAC \\
\hline & $\begin{array}{l}\text { Epigonus } \\
\text { telescopus }\end{array}$ & No TAC \\
\hline & $\begin{array}{l}\text { Galeus } \\
\text { melanostomus }\end{array}$ & No TAC \\
\hline & $\begin{array}{l}\text { Hippoglossus } \\
\text { hippoglossus }\end{array}$ & No TAC \\
\hline & $\begin{array}{l}\text { Molva } \\
\text { macropthalma }\end{array}$ & No TAC \\
\hline
\end{tabular}




\begin{tabular}{|c|c|c|}
\hline & Mora moro & No TAC \\
\hline & $\begin{array}{l}\text { Mustelus } \\
\text { mustelus/asterias }\end{array}$ & No TAC \\
\hline & Phycis blennoides ${ }^{2}$ & $\begin{array}{l}\text { TACs enforce (up to 2017-2018), removed from TAC } \\
\text { management from } 2019\end{array}$ \\
\hline & $\begin{array}{l}\text { Polyprion } \\
\text { americanus }\end{array}$ & No TAC \\
\hline & Scorpaena scrofa & No TAC \\
\hline & $\begin{array}{l}\text { Scyliorhinus } \\
\text { canicula }\end{array}$ & No TAC \\
\hline & $\begin{array}{l}\text { Scyliorhinus } \\
\text { stellaris }\end{array}$ & No TAC \\
\hline & $\begin{array}{l}\text { Synaphobranchus } \\
\text { kaupi }\end{array}$ & No TAC \\
\hline & $\begin{array}{l}\text { Torpedo } \\
\text { marmorata }\end{array}$ & No TAC \\
\hline & Torpedo nobiliana & No TAC \\
\hline \multirow[t]{3}{*}{$\begin{array}{l}\text { TAC applied at } \\
\text { the level of } \\
\text { Order }\end{array}$} & $\begin{array}{l}\text { Amblyraja } \\
\text { hyperborea }\end{array}$ & Included within the TACs for 'skates and rays' \\
\hline & $\begin{array}{l}\text { Dipturus } \\
\text { oxyrinchus }\end{array}$ & Included within the TACs for 'skates and rays' \\
\hline & $\begin{array}{l}\text { Leucoraja } \\
\text { circularis }^{1}\end{array}$ & $\begin{array}{l}\text { Included within the TACs for 'skates and rays'. Must be } \\
\text { reported by species in Subarea } 6 \text { and Divisions 7.a-c, 7.e- } \\
\text { k. }\end{array}$ \\
\hline
\end{tabular}




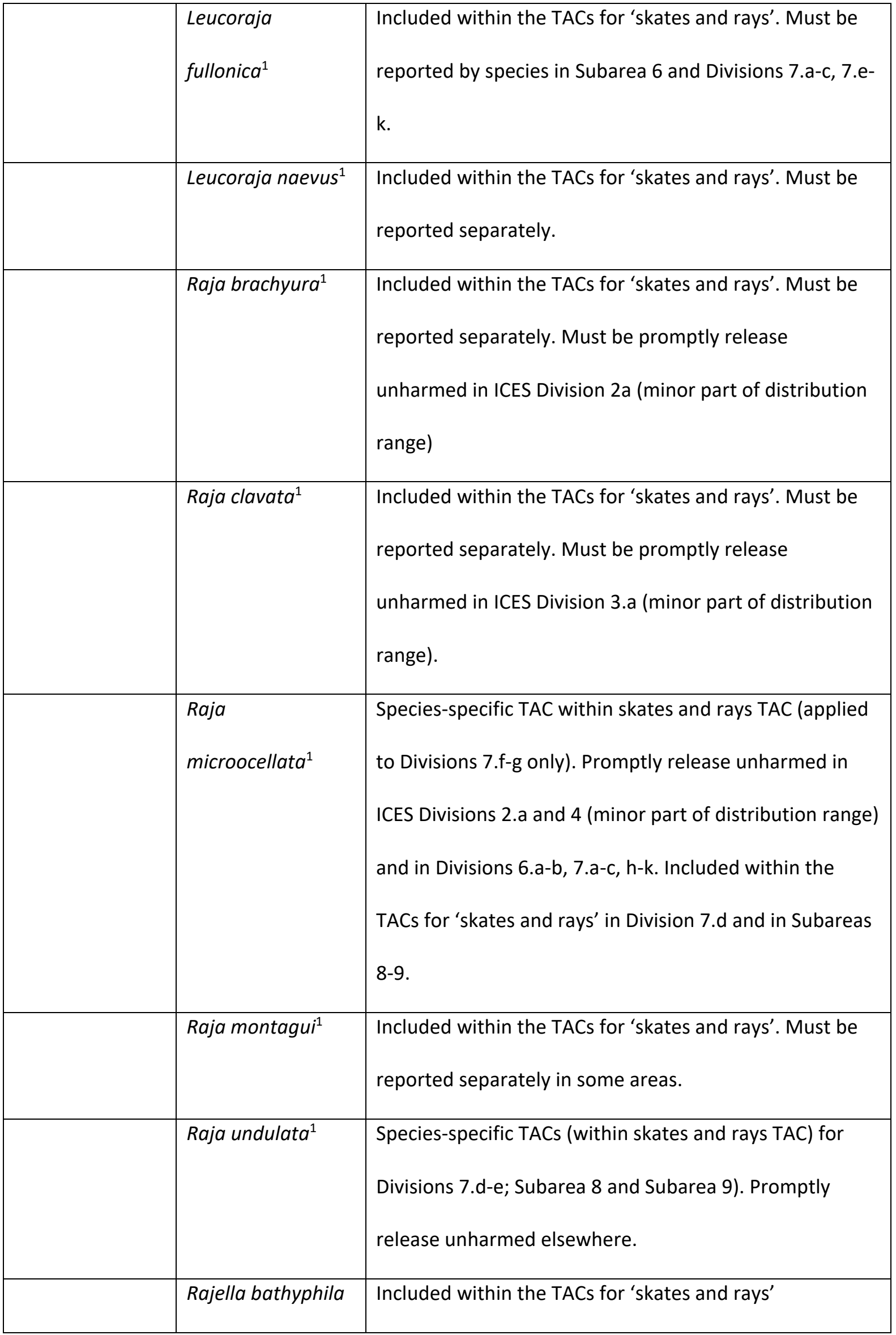




\begin{tabular}{|c|c|c|}
\hline & Rajella fyllae & Included within the TACs for 'skates and rays' \\
\hline & Rajella lintea & Included within the TACs for 'skates and rays' \\
\hline \multirow[t]{5}{*}{$\begin{array}{l}\text { TAC applied at } \\
\text { the genus-level } \\
\text { or family level }\end{array}$} & $\begin{array}{l}\text { Lepidorhombus } \\
\text { whiffiagonis }^{1}\end{array}$ & TACs for Lepidorhombus spp. \\
\hline & $\begin{array}{l}\text { Lophius } \\
\text { budegassa }^{1}\end{array}$ & TACs for anglerfish Lophiidae \\
\hline & $\begin{array}{l}\text { Lophius } \\
\text { piscatorius }^{1}\end{array}$ & TACs for anglerfish Lophiidae \\
\hline & $\begin{array}{l}\text { Scophthalmus } \\
\text { rhombus }^{1}\end{array}$ & $\begin{array}{l}\text { TAC for Scophthalmus rhombus and S. maximus (EU } \\
\text { waters of Division 2.a and Subarea } 4 \text { only) }\end{array}$ \\
\hline & Sebastes spp. ${ }^{1}$ & TAC for Sebastes spp. \\
\hline \multirow[t]{4}{*}{$\begin{array}{l}\text { Species-TACs by } \\
\text { management } \\
\text { area(s) }\end{array}$} & Brosme brosme $^{1}$ & TACs in place \\
\hline & $\begin{array}{l}\text { Coryphaenoides } \\
\text { rupestris }^{2}\end{array}$ & $\begin{array}{l}\text { Precautionary TACs for 3; 5b, 6-, 7; and 8, 9, 10, } 12 \text { and } \\
\text { 14. Combined TAC for grenadiers in Greenland and Arctic } \\
\text { waters. No TAC in other areas. }\end{array}$ \\
\hline & Molva dypterygia ${ }^{1}$ & TACs in place \\
\hline & Molva molva & TACs in place \\
\hline \multirow[t]{2}{*}{$\begin{array}{l}\text { Conservative } \\
\text { management }\end{array}$} & Lamna nasus $^{1}$ & $\begin{array}{l}\text { Prohibited species, to be promptly released unharmed } \\
\text { when caught (all waters) }\end{array}$ \\
\hline & $\begin{array}{l}\text { Macrourus } \\
\text { berglax }{ }^{2}\end{array}$ & $\begin{array}{l}\text { No target fisheries. Landings of upto } 1 \% \text { of the TAC for } \\
\text { Coryphaenoides rupestris. }\end{array}$ \\
\hline
\end{tabular}




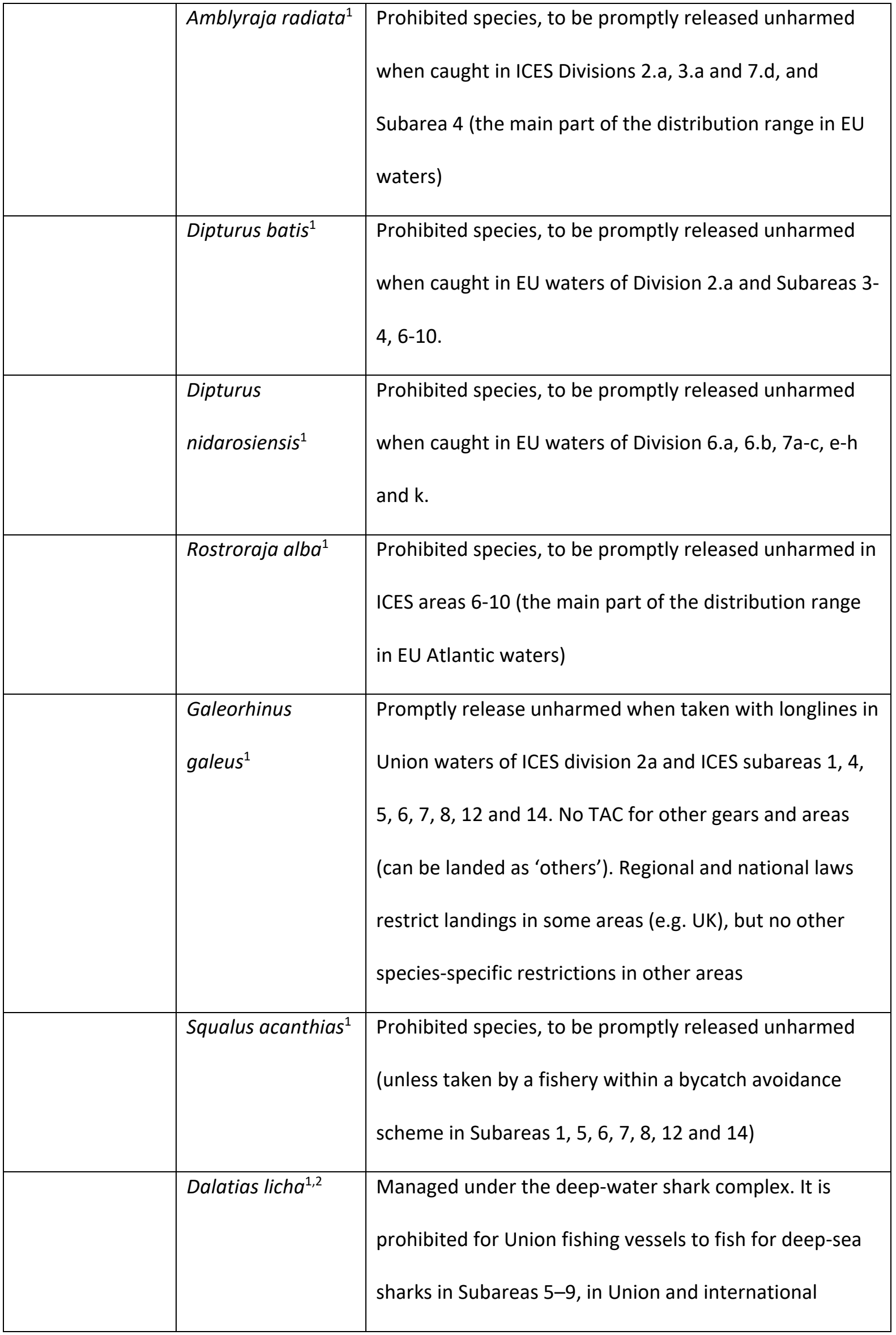




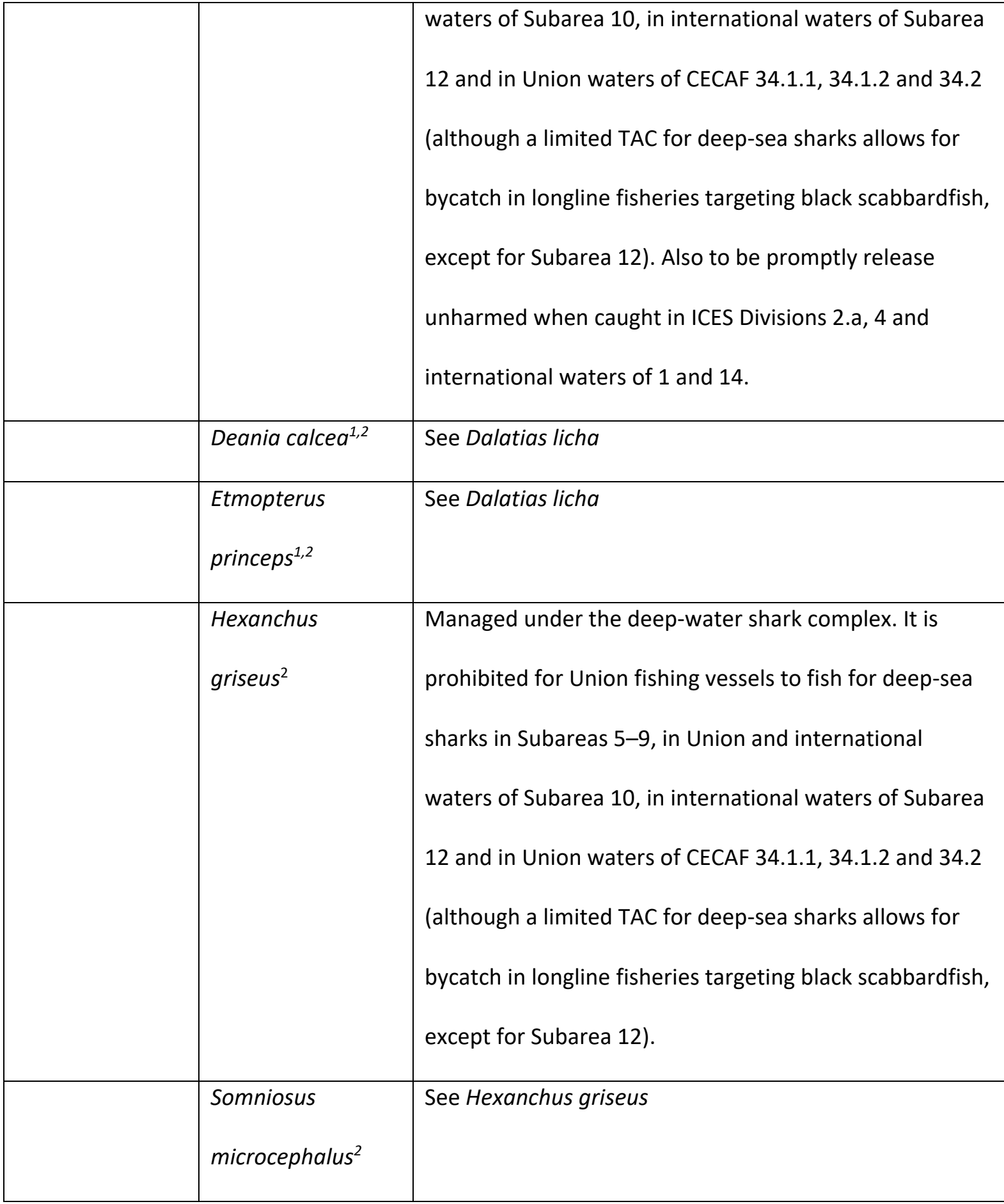

${ }^{1}$ EU 2018a. COUNCIL REGULATION (EU) 2019/124 of 30 January 2019 fixing for 2019 the fishing opportunities for certain fish stocks and groups of fish stocks, applicable in Union waters and, for Union fishing vessels, in certain non-Union waters.

${ }^{2}$ EU 2018b. Council Regulation (EU) 2018/2025 of 17 December 2018 fixing for 2019 and 2020 the fishing opportunities for Union fishing vessels for certain deep-sea fish stocks.Supplementary figures 
Amblyraja radiata

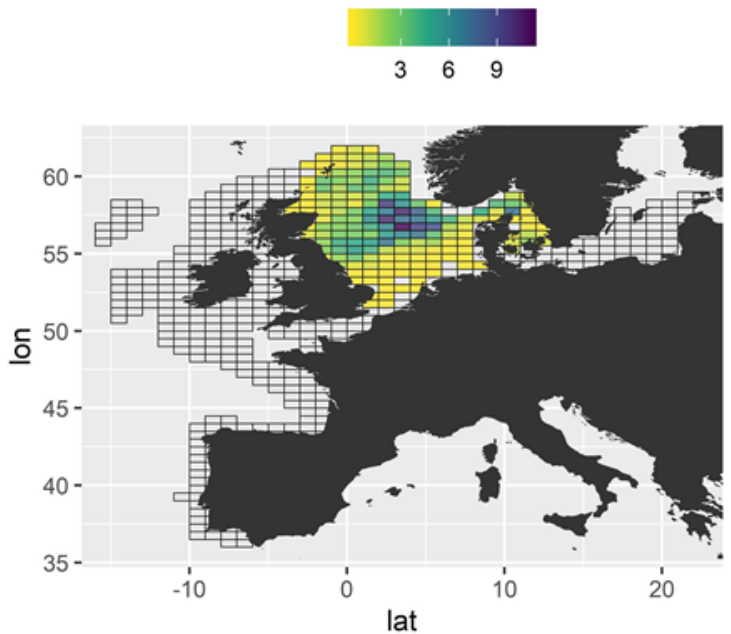

Brosme brosme

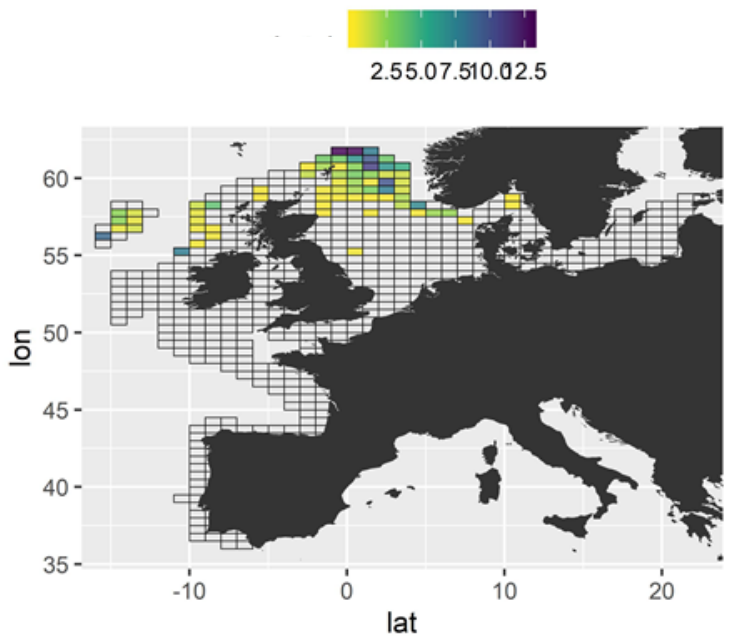

Conger conger
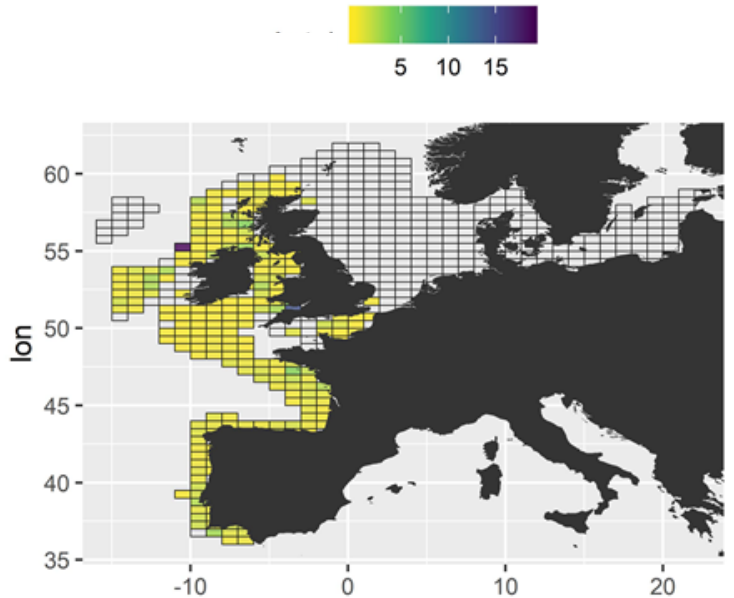

lat
Anarhichas lupus
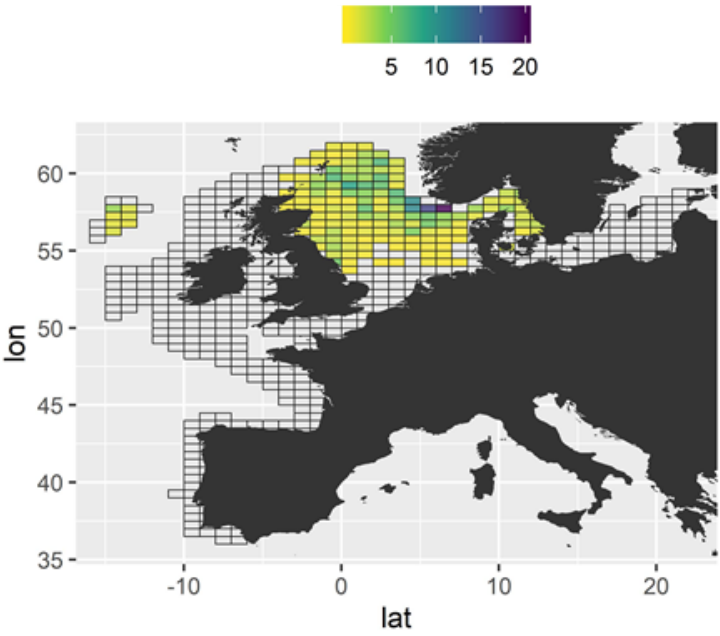

Chimaera monstrosa

$\begin{array}{lll}2.5 & 5.0 & 7.5\end{array}$

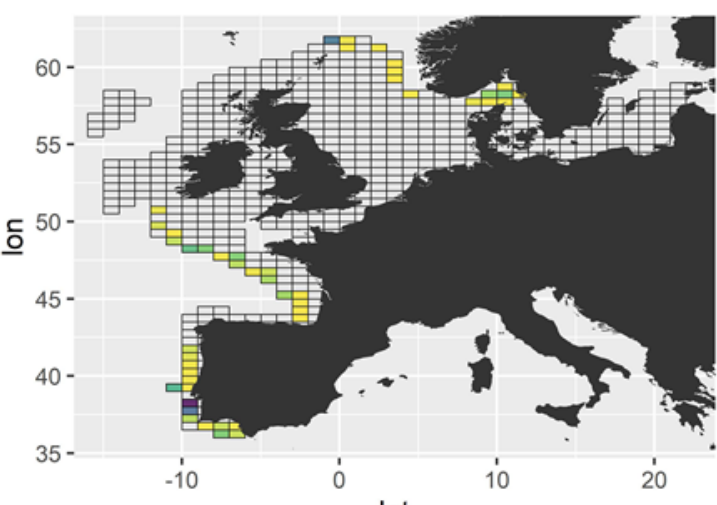

Dasyatis pastinaca
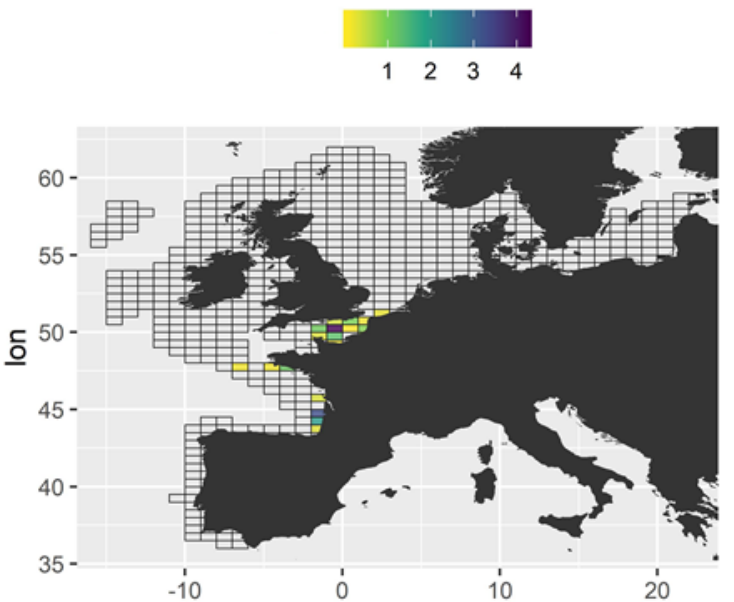

lat

Figure S1. Spatial distribution of sensitive species. 


\section{Dipturus spp}

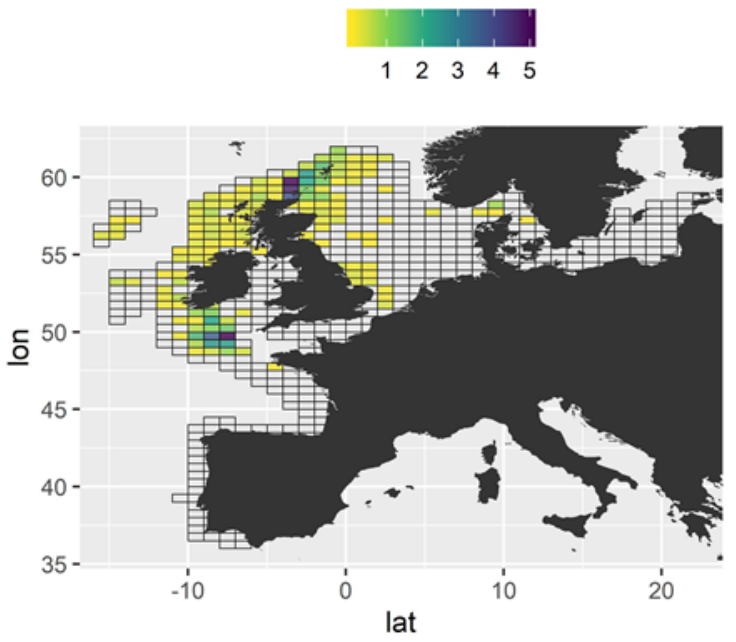

Galeus melastomus
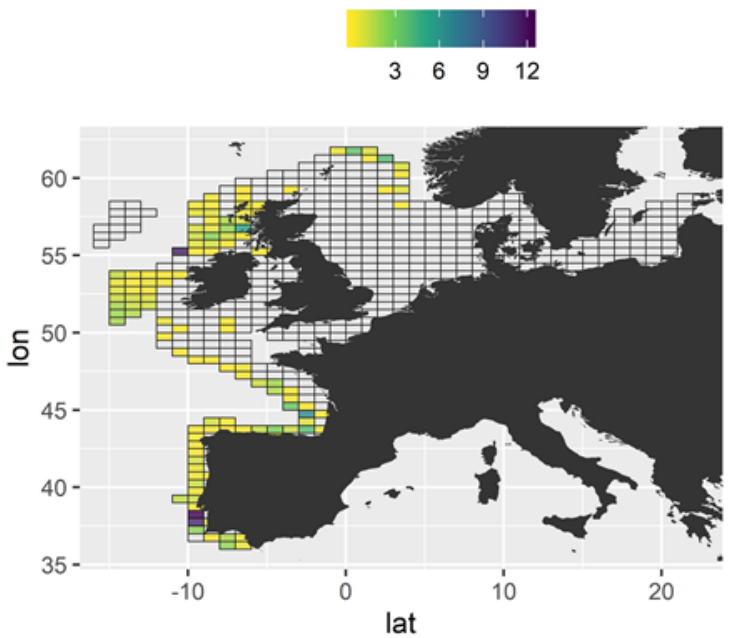

Hippoglossus hippoglossus
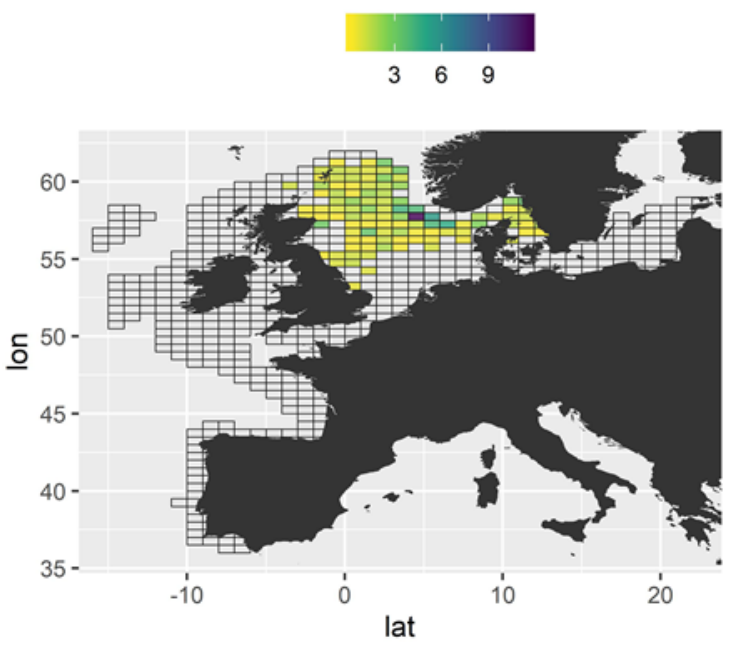

Galeorhinus galeus
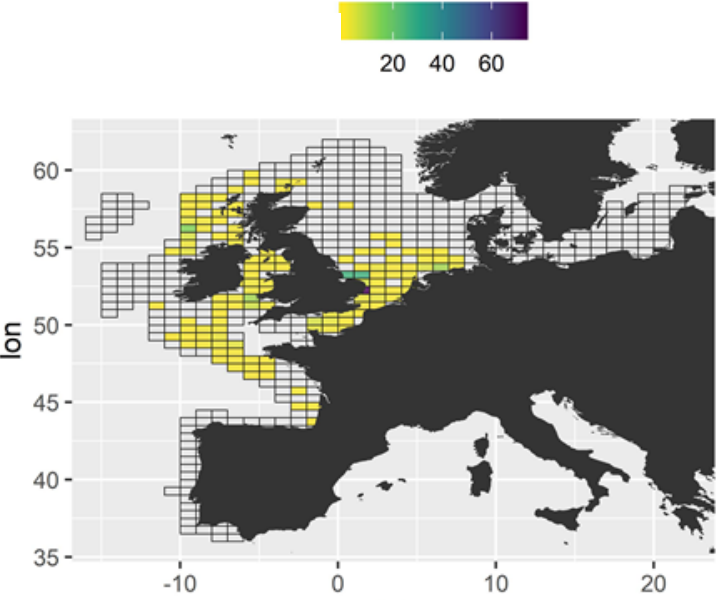

lat

Hexanchus griseus
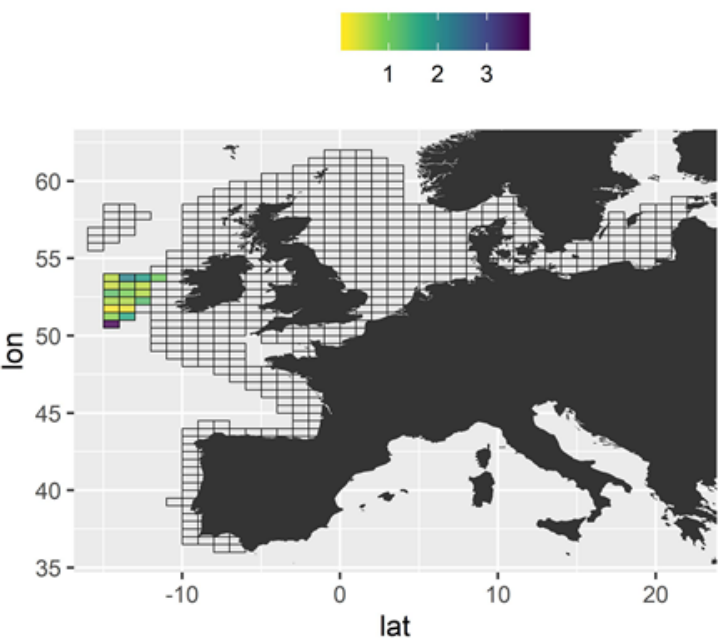

Leucoraja circularis
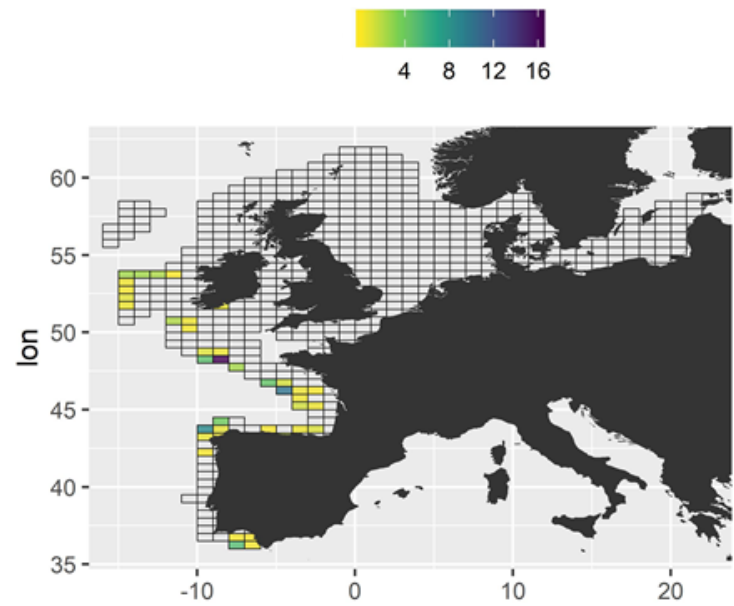

lat

Figure S1. Spatial distribution of sensitive species continued. 
Leucoraja fullonica
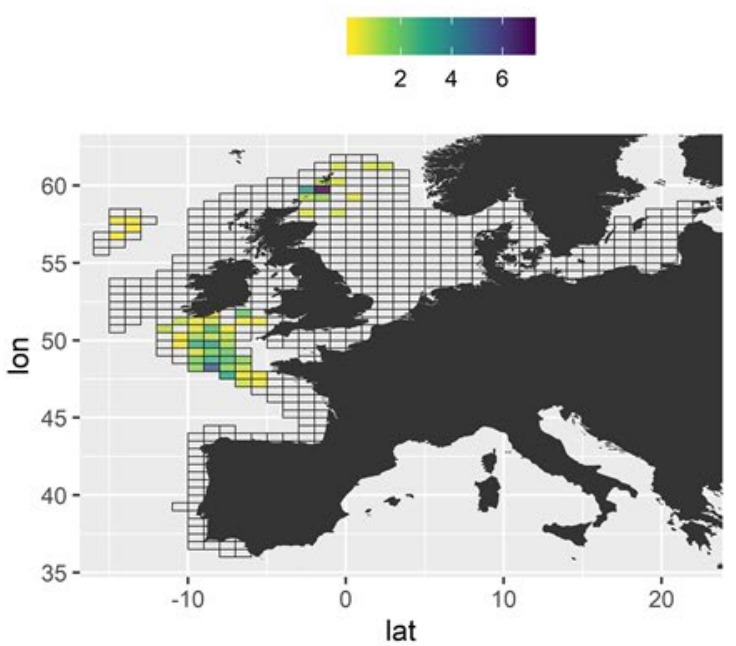

Lophius budegassa

122345

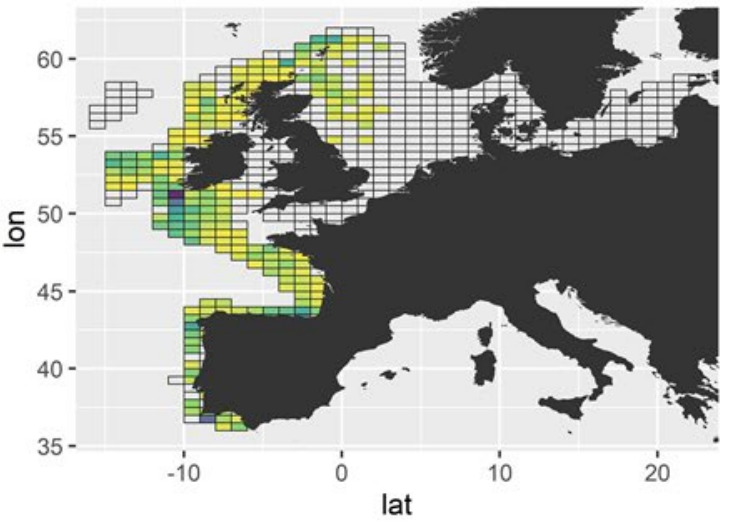

Molva macropthalma
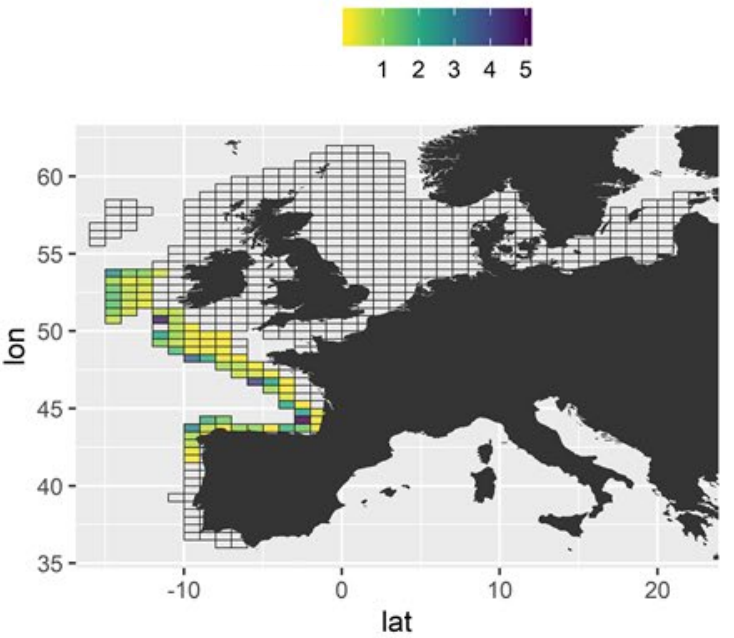

Leucoraja naevus

2.55 .07 .5

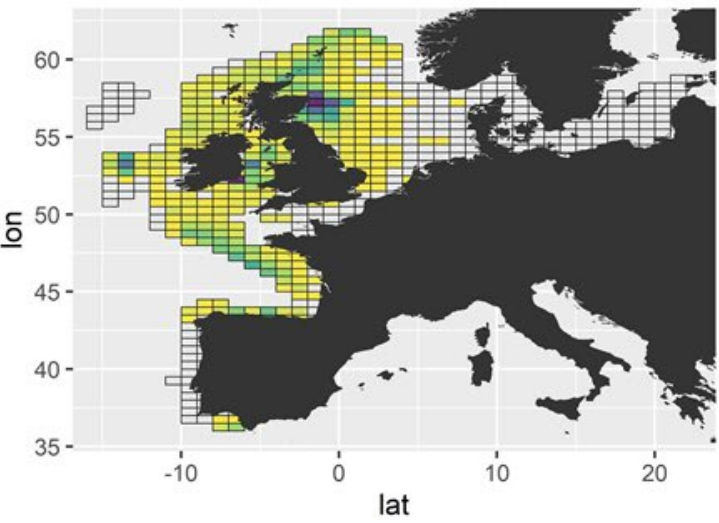

Lophius piscatorius
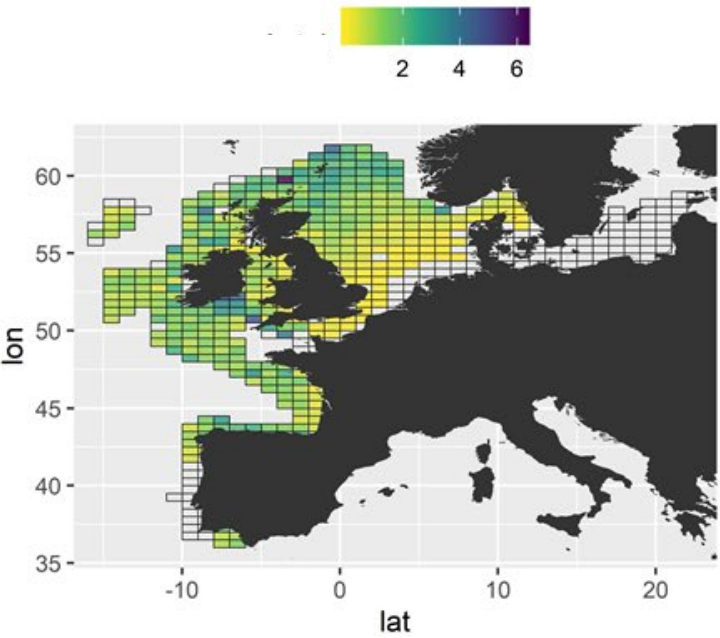

Molva molva
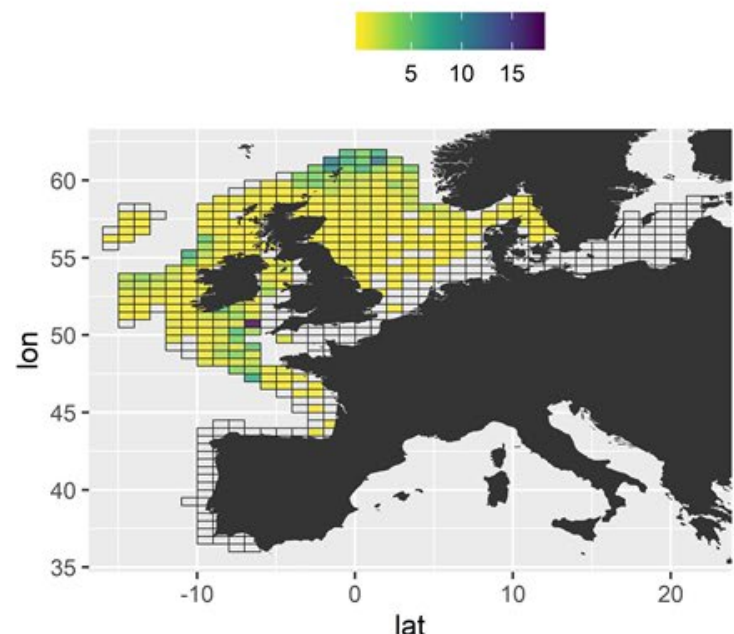

Figure S1. Spatial distribution of sensitive species continued. 
Mustelus spp

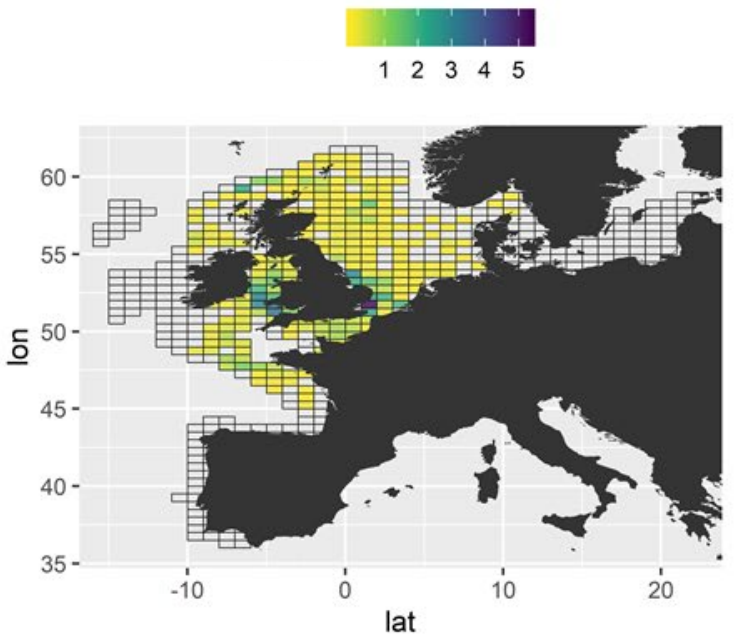

Raja brachyura

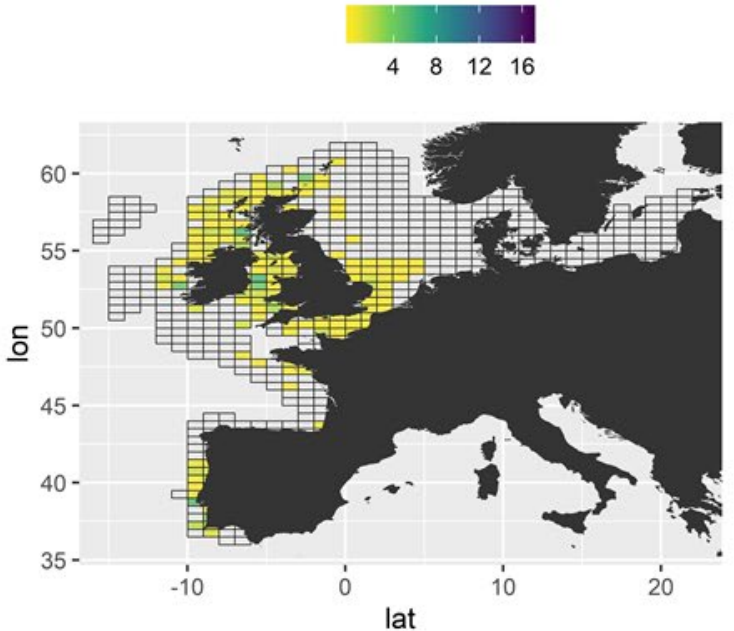

Raja microocellata
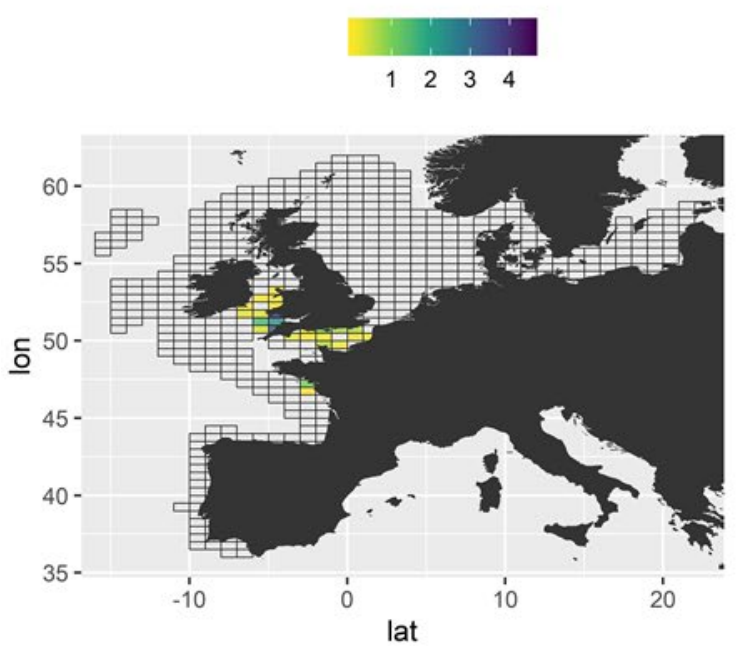

Phycis blennoides
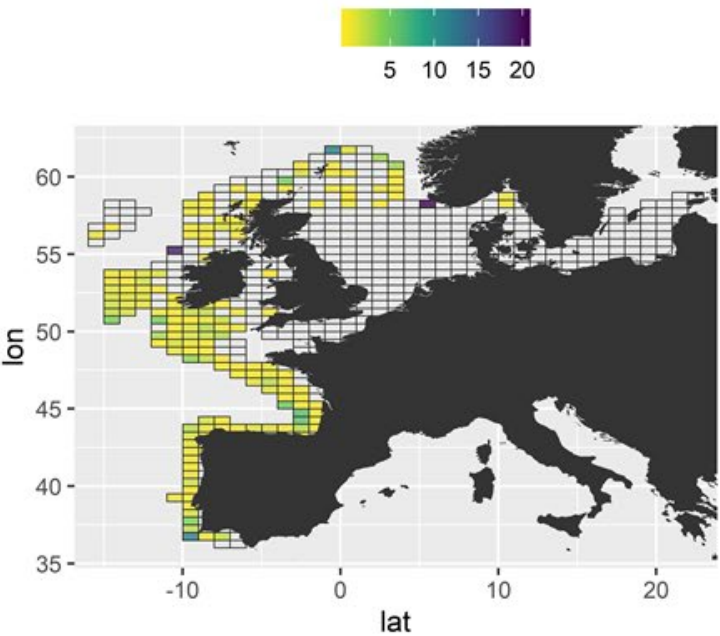

Raja clavata

$102030 \quad 40$

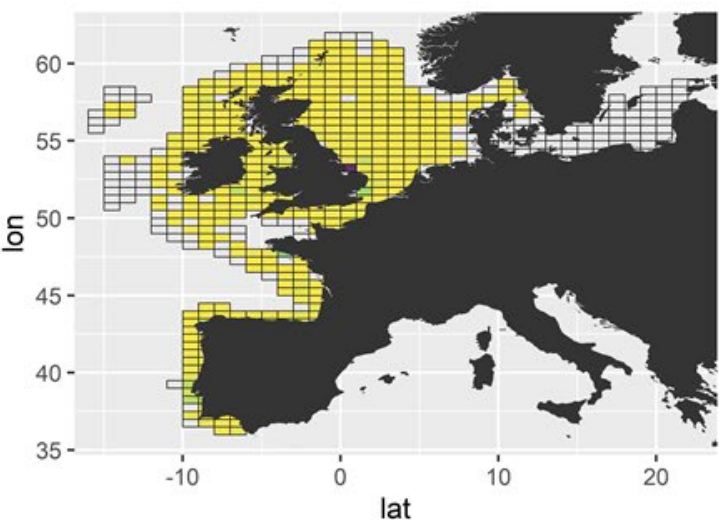

Raja montagui
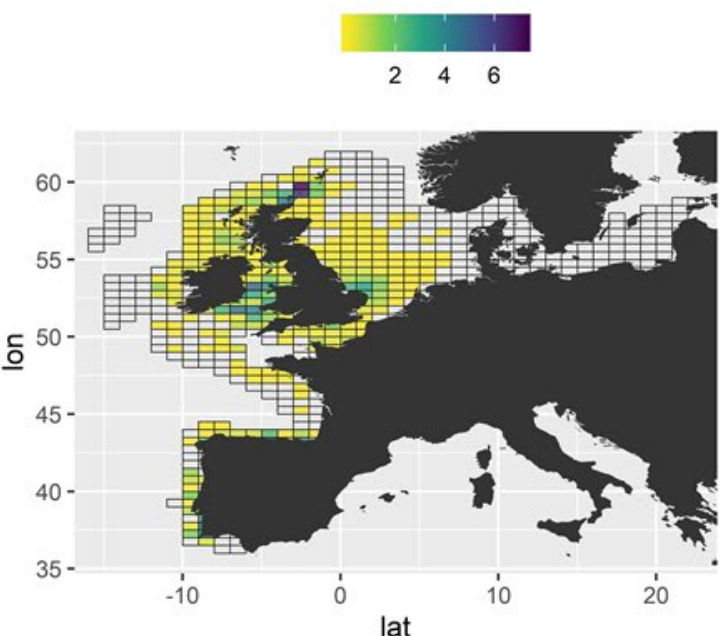

Figure S1. Spatial distribution of sensitive species continued. 
Raja undulata
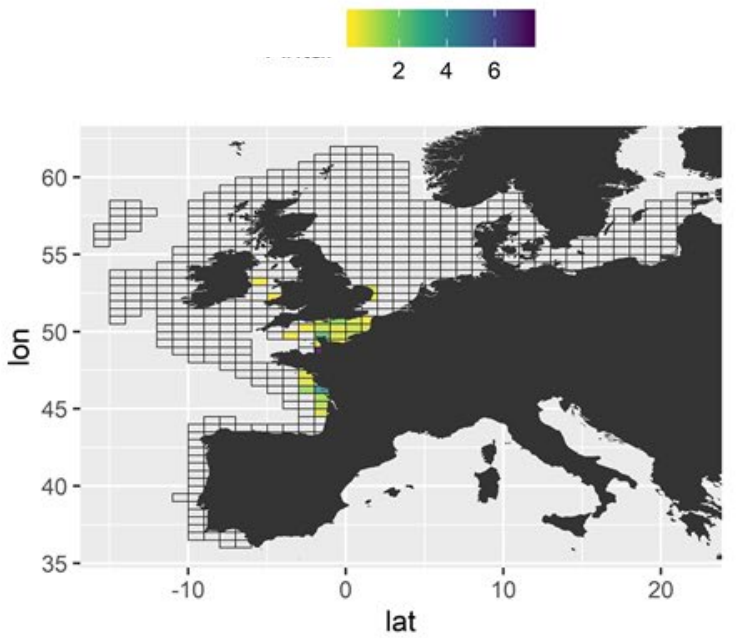

Scyliorhinus canicula
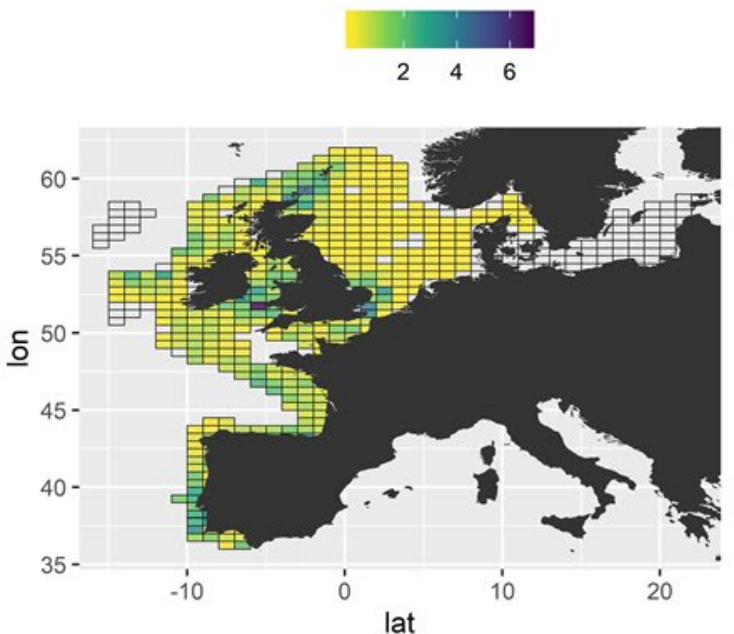

Squalus acanthias
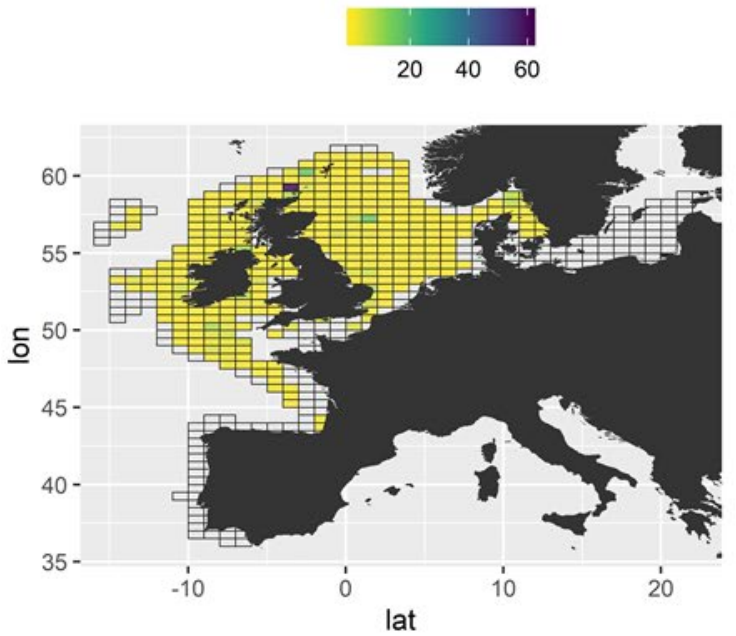

Scophthalmus rhombus

2.55 .07 .510 .0

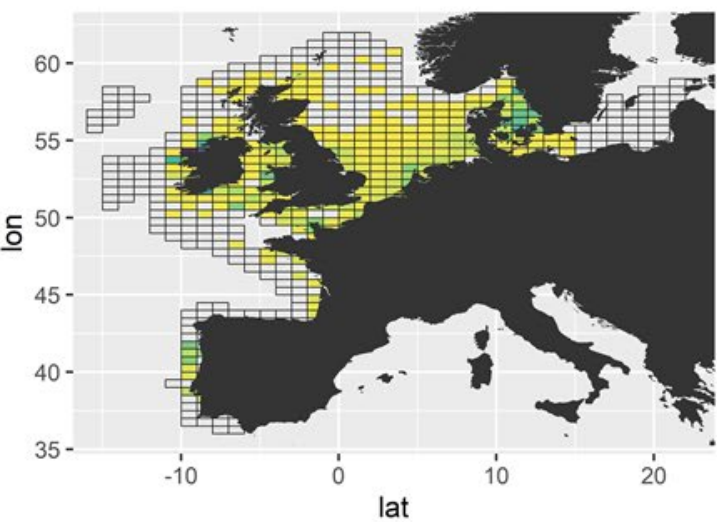

Scyliorhinus stellaris
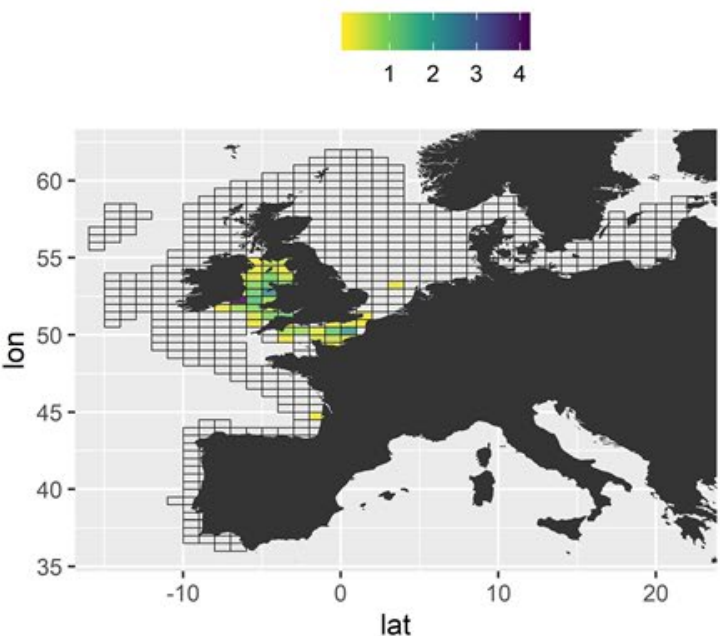

Torpedo marmorata
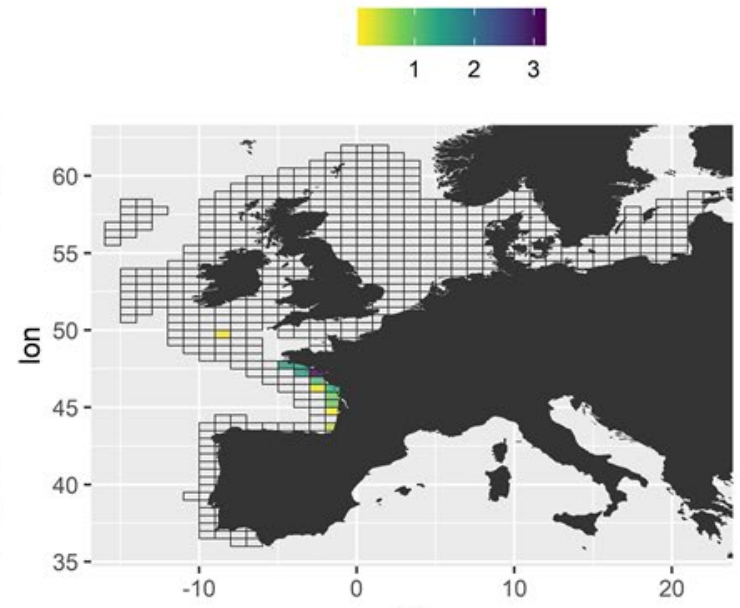

lat

Figure S1. Spatial distribution of sensitive species continued. 
